\title{
Excavation of Neopalatial deposits at Tholos (Kastri), Kythera
}

\author{
A. Bevan, E. Kiriatzi, C. Knappett, E. Kappa, S. Papachristou \\ Postprint of a 2002 paper in the Annual of the British School at Athens 97: 55-96.
}

\begin{abstract}
Several rock-cut features, exposed on the surface of a trackway in the Tholos area of Kastri, Kythera, were excavated in July-August 2000 as a synergasia between Kythera Island Project and 2nd EPCA. Although the surviving deposits were extremely shallow, they produced large quantities of conical cups and other pottery of Late Minoan I date. Further comparative analysis of the features themselves and their finds suggests that these are the remains of tomb chambers similar to those excavated in the area in the 1960s. These tombs and their assemblages show extremely strong cultural connections with Crete, but also idiosyncrasies that probably reflect the particular mortuary customs of the island.
\end{abstract}

\section{Introduction}

The site of Kastri on Kythera has become an important example of the expansion of Cretan (Minoan) material culture beyond Crete in the later third and second millennium BC. ${ }^{1}$ The first identification of Minoan finds on the island was made by Benton, who noted tombs and ceramics of this date on and around the Kastri

\footnotetext{
${ }^{1}$ The excavation was a synergasia between the Kythera Island Project and 2nd EPCA (co-directed by C. Broodbank and A. Tsaravopoulos respectively). We thank the Central Archaeological Council and the Ministry of Culture for the permit to conduct this work. Funding was provided by the Institute for Aegean Prehistory (INSTAP), the British School at Athens and the Mediterranean Archaeological Trust. The field team consisted of A. Bevan (field director, University College London [UCL]), A. Brysbaert (on-site conservator, University of Glasgow), S. Diamantopoulou (Institute of Education, London), V. Isaakidou (bioarchaeologist, UCL), E. Kappa (University of Ioannina), S. Papachristou (University of Athens) and E. Twigger (UCL), with total station support from J. Conolly (UCL) and O. Pryce (UCL). The pottery was studied by E. Kiriatzi (Fitch Laboratory, BSA) and C. Knappett (University of Cambridge) with the assistance of E. Kappa. D. Romanou (UCL) and M. Nikiforidou (T.E.I. Athens) drew the pottery. We would like to thank INSTAP and T. Brogan for lending the services of the INSTAP (East Crete) conservator, M. Rogenbuche, to whom we are also grateful for mending most of the non-conical cup vessels. The authors are especially grateful to C. Broodbank and A. Tsaravopoulos for guidance and continuous logistical support during the excavation and subsequent study of the Tholos material. The latter took place at the Archaeological Museum of Peiraias and we would like to thank K. Axioti and all the staff of the Museum for facilitating our study. L. Preston provided advice and information on Cretan burial practices and C. Frederick and N. Krahtopoulou onsite geoarchaeological advice. Thanks also go to C. Inepekoglou for donating spraying equipment for the excavation. Finally, we would like to express our particular gratitude to C. Broodbank, without whose input and enabling of every stage of the excavation and post-excavation process this report would not be possible.

Special Abbreviation:

Kythera $=$ J.N. Coldstream, \& G.L. Huxley, Kythera. Excavations and Studies (London, 1972).
} 
headland on the eastern coast. ${ }^{2}$ Subsequent survey and excavation did much to confirm these initial impressions and to demonstrate the existence of a large settlement at Kastri along with an impressive number of Neopalatial tombs. ${ }^{3}$ The significance of this site was also emphasised by the discovery and excavation during the 1990s of a peak sanctuary on the nearby summit of Agios Georgios. ${ }^{4}$ Despite this, in the thirty years since the publication of Coldstream and Huxley's investigations, little new light has been shed on Kastri itself, either through excavation or surface investigations.

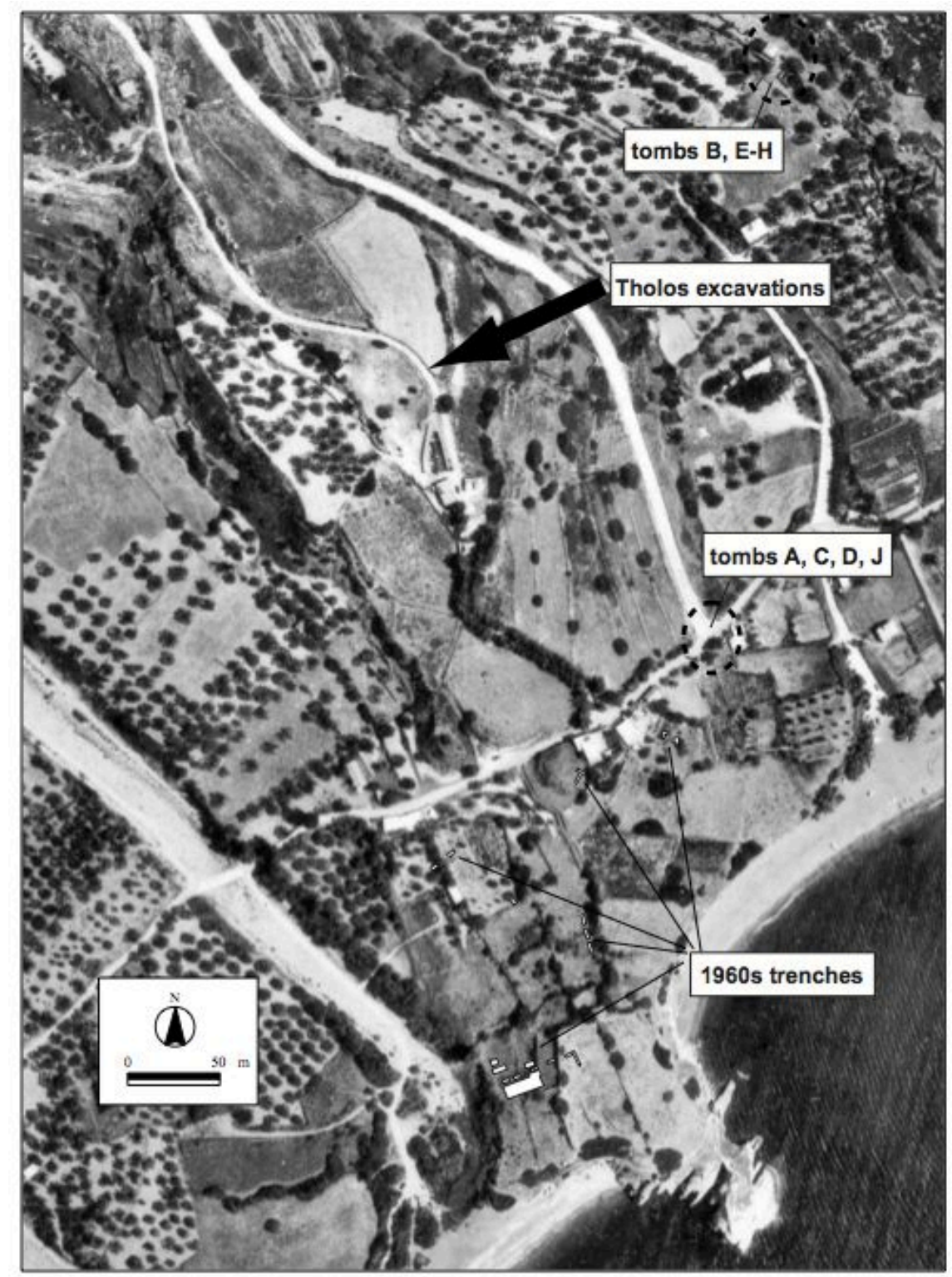

Fig.1 Aerial photo of Kastri and environs showing the location of the Tholos excavations and the 1960s trenches and tombs.

\footnotetext{
${ }^{2}$ S. Benton, 245-6; 'The Ionian Islands', BSA 32 (1931-2), 213-46.

${ }^{3}$ See H. Waterhouse \& R. Hope Simpson, 148-60; 'Prehistoric Laconia: Part II', BSA 56 (1961), $114-$ 75 , for an early survey of the site and its tombs. Kythera for publication of the 1960s excavations by Coldstream and Huxley at Kastri.

${ }^{4}$ Y. Sakellarakis, 'Minoan religious influence in the Aegean: the case of Kythera', BSA 91 (1996), 8199.
} 
This report therefore offers significant new information on the Neopalatial period at the site that emerged during a short excavation conducted from $24^{\text {th }}$ July to $2^{\text {nd }}$ August 2000. It was undertaken within the wider context of a new phase of surface survey and documentation of the site by the Kythera Island Project (KIP). ${ }^{5}$ In the summer of 1999 , as part of these investigations, a large amount of whole and fragmentary pottery was discovered in an exposed and partially truncated condition along the surface of c. $20 \mathrm{~m}$ of dirt track running up the ridge that forms the landward extension of Kastri (an area called 'Tholos' by locals, see FIG. 1). Survey work in that year established that this entire zone was part of the $2^{\text {nd }}$ millennium site. Investigation by 2nd EPCA in 1999 established that these vessels were situated within a series of rock-cut features. This exposed material was threatened by erosion of the track surface from rainwater run-off and regular bulldozing as part of road maintenance. The following discussion sets out the findings of the subsequent excavation and is divided into four parts, addressing a) the excavation methodology, b) each of the excavated features and its assemblage, c) the Tholos ceramics and their parallels, and d) the overall interpretation of the features and their finds.

\section{Method}

The extreme hardness of the ground and the time available limited overall cleaning of the road surface. Following regular spraying to soften this surface, excavation concentrated on one main area, centred on two rectangular cuttings, but a few similar features nearby were also explored in a number of smaller tests (FIG. 2). This strategy investigated all recognisable features and visible pottery.

A context-based recording system was used to document the excavation. Features were recorded as a single numbered series of depositional episodes i.e. cuts, fills or layers - and the numbers referred to in the text below reflect deposits grouped into discrete features under their original cut number. A brief summary of the (limited) stratigraphy and of the numbered cuts and fills relevant to each feature are provided at the end. In contrast, the unique sequence of numbers allocated to finds in the field has necessarily been subject to significant alteration during post-excavation study. Finds have therefore been renumbered for publication here; a concordance with the original field sequence is provided with each catalogue entry and is listed in reverse at the end of this report.

Most excavated features turned out to be preserved to an extremely shallow depth (10-25cms) and the volume of the surviving deposits was consequently minute (c. 3 cubic metres was excavated in total). Apart from a few obsidian flakes (which are ubiquitous at modest densities over this part of the ridge, and probably earlier in date), a single piece of copper/bronze wire and one possible groundstone pounder, all the cultural material encountered was ceramic, comprising over 250 vessels (whole or large fragments). Partly as a result of the

\footnotetext{
5 C. Broodbank, 'Kythera Survey: Preliminary Report on the 1998 Season', BSA 94 (1999), 191-214. See also D. Blackman, 'Archaeology in Greece 1999-2000', Archaeological Reports 46 (2000), 23; Blackman, Arch Reps 47 (2001), 20.
} 
thinness and exposed nature of the deposits, preservation was poor and much of the pottery was quite degraded. ${ }^{6}$ Deposits were excavated using hand picks, trowels and, where pottery concentrations were high, leaf trowels and dentistry tools. Some pottery groups were removed as blocks in plaster by the on-site conservator and subsequently separated during conservation and study in the Peiraias Museum. ${ }^{7}$

No animal or human bone was recovered during the excavation itself. In addition, all excavated deposits were sampled for bioarchaeological remains and c. 1200 litres of soil were collected for processing. Of this total, 215 litres $(50 \%$ of the larger contexts) were dry-sieved using a $1 \mathrm{x} 1 \mathrm{~cm}$ mesh, after a sub-sample had been processed by bucket flotation. ${ }^{8}$ The latter method was also used for a representative subset of other features. The float was poured into two sieves of $1 \mathrm{~mm}$ and $300 \mu$. Neither process yielded any bioarchaeological (bone or charred plant) remains. ${ }^{9}$ Two possible explanations can be put forward for their absence: either such material was never deposited in the excavated features in the first place or it has since been completely destroyed by post-depositional processes.
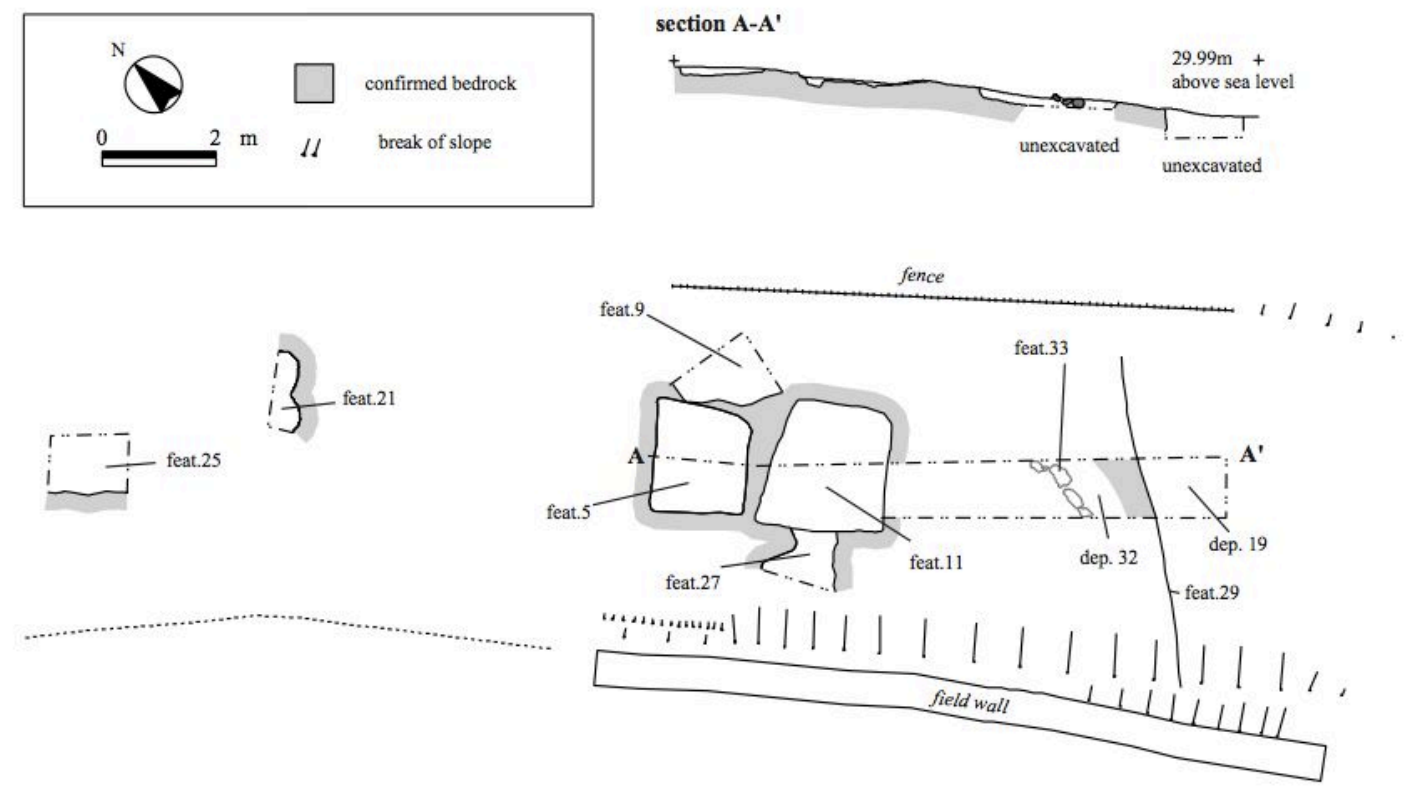

Fig.2 Overall site plan of Tholos excavations.

\footnotetext{
${ }^{6}$ In all cases, the vessel surfaces were very poorly preserved and fabrics were soft and fragile even when they were obviously highly fired.

${ }^{7}$ It proved necessary to clean the pottery in a weak acid solution followed by prolonged rinsing; prior to this, ten sherds had been removed for possible future organic residue analysis.

${ }^{8}$ The method was dictated by the absence of a flotation machine and the limited amount of soil processed was due to water shortage experienced that year on the island.

${ }^{9}$ The remaining soil samples are currently stored in the KIP apotheke (Mitata, Kythera) for further processing, although on present evidence it is thought unlikely that they contain any bioarchaeological material.
} 


\section{Excavated Features}

The excavated features were all cut into a light yellowish-brown, marly limestone bedrock (FIG. 3). Most of the track surface was in fact bedrock of this kind, sometimes with a thin layer of light brown silty clay overburden, but often fully exposed as the result of repeated bulldozing of the roadway in the process of its upkeep. This appears to be only the most modern phase of maintenance of the roadway (running along a natural ridge route) which, while perhaps shifting in exact location, was probably in use from the beginning of settlement in the area as a route to the island's interior. ${ }^{10}$

Feature 5 (FIGS. 4-6, PLATE 2c)

Feature $5(180 \times 160 \mathrm{~cm}$, max. depth $18 \mathrm{~cm})$ was the best preserved of the excavated features. It was roughly rectangular with a generally flat base (dug at a slight incline into the slope) and near vertical sides. It has one main infilling episode of reddish-brown silty clay. ${ }^{11}$ This is quite different from the existing surface soil of the top of the ridge and, in terms of geomorphology, appears to have formed over a long period of time (C. Frederick, pers.comm.). In other words, it is likely to represent remnants of older soils preserved in the rock cuttings, but lost to erosion elsewhere on the ridge.

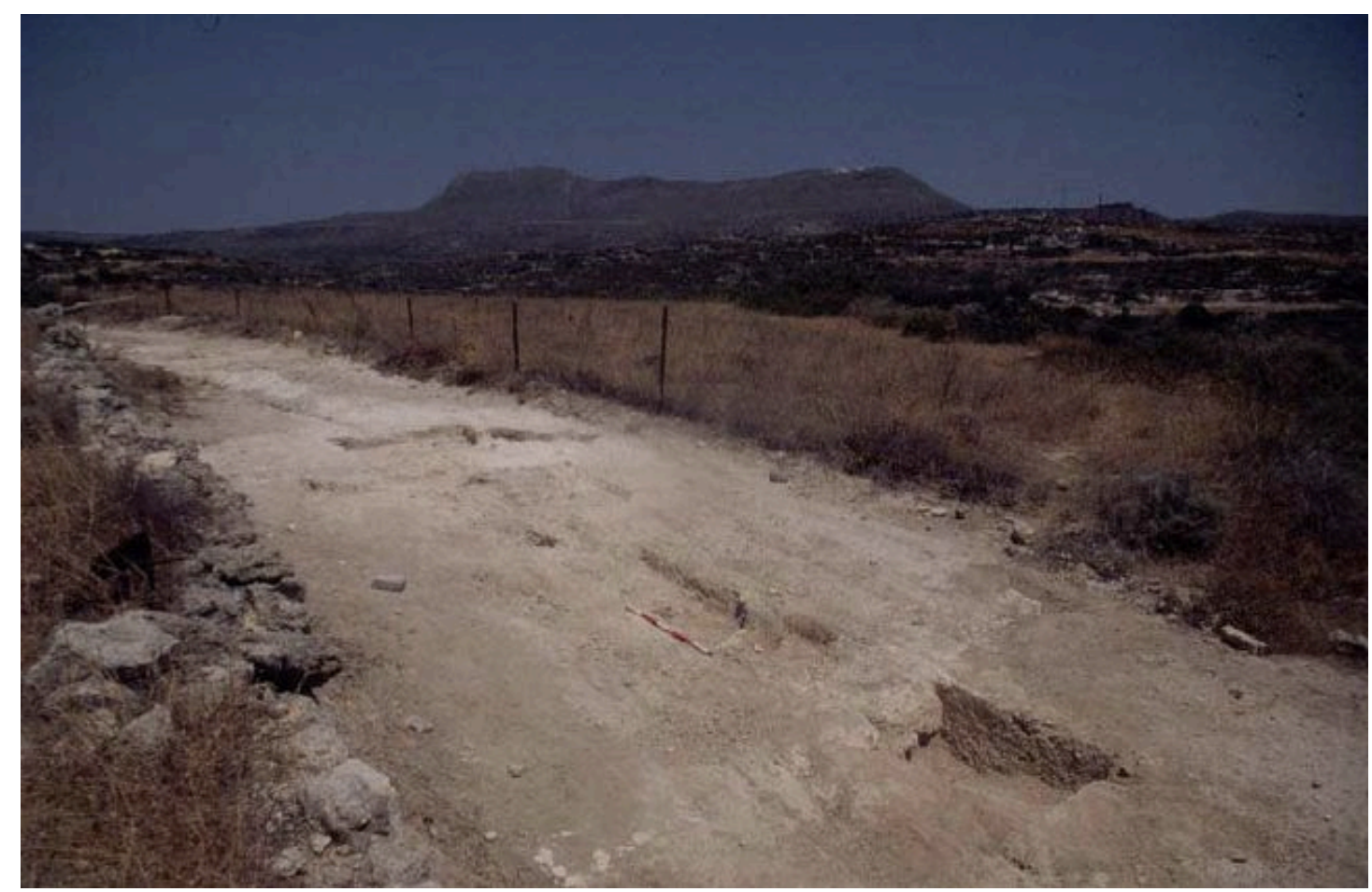

Fig.3 View of track and excavation area looking north

The finds were impressive given the small volume of fill excavated. A total of 139 catalogued vessels (whole or large fragments) came from feature 5, of which 122 were conical cups. The rest included a range of types: hemispherical cups (2),

\footnotetext{
${ }^{10}$ G.N. Leontsinis, 203-204, 228; The Island of Kythera. A Social History (Athens, 1987).

${ }^{11}$ Within this fill were some red clay inclusions, some of which may have been degraded pottery.
} 
tumbler (1), kalathos (1), ring-handled basins (2), conical bowl (1), blossom bowl (1), low-spouted jugs (2), ewers (2), jug/ewer (1), globular jars (2?) with lid (1) and closed vessel of indeterminate type (1). Most of the conical cups were found together, either placed on their side in tight stacks or inverted on top of a large (highly degraded) conical bowl (7; FIGS. 5, 9). All of these were placed on a bed of water-worn pebbles c. $10 \mathrm{~cm}$ thick in the southern corner of the feature. Similar pebbles were also found incrusted to the interior of one jug (10).12 This jug was found in the middle of the feature with a group of other whole and fragmentary vessels that, judging by their discrete sub-rectangular arrangement in the ground, may originally have been placed there in a box or bag that has since disappeared (FIGS. 5, 10). The copper/bronze wire fragment (PLATE 2a) found nearby may conceivably have been associated with this group (as a fastener?). Moreover, a number of undiagnostic sherds (c. 140), most of them in the same fabric, were found in connection with the above group of pots in the central area of feature $5 .{ }^{13}$ They were too worn to be assigned to a specific vessel type but the majority seem to belong to a single highly degraded pot (possibly a large conical bowl or brazier?).

In addition, a number of small, quite abraded sherds (c. 600-700) were recovered from the fill of feature 5. Most of them, c. 500 tiny fragments, related to conical cups of the same thick-walled type as the rest in feature 5 (fragments of at least 10 more bases were noted).

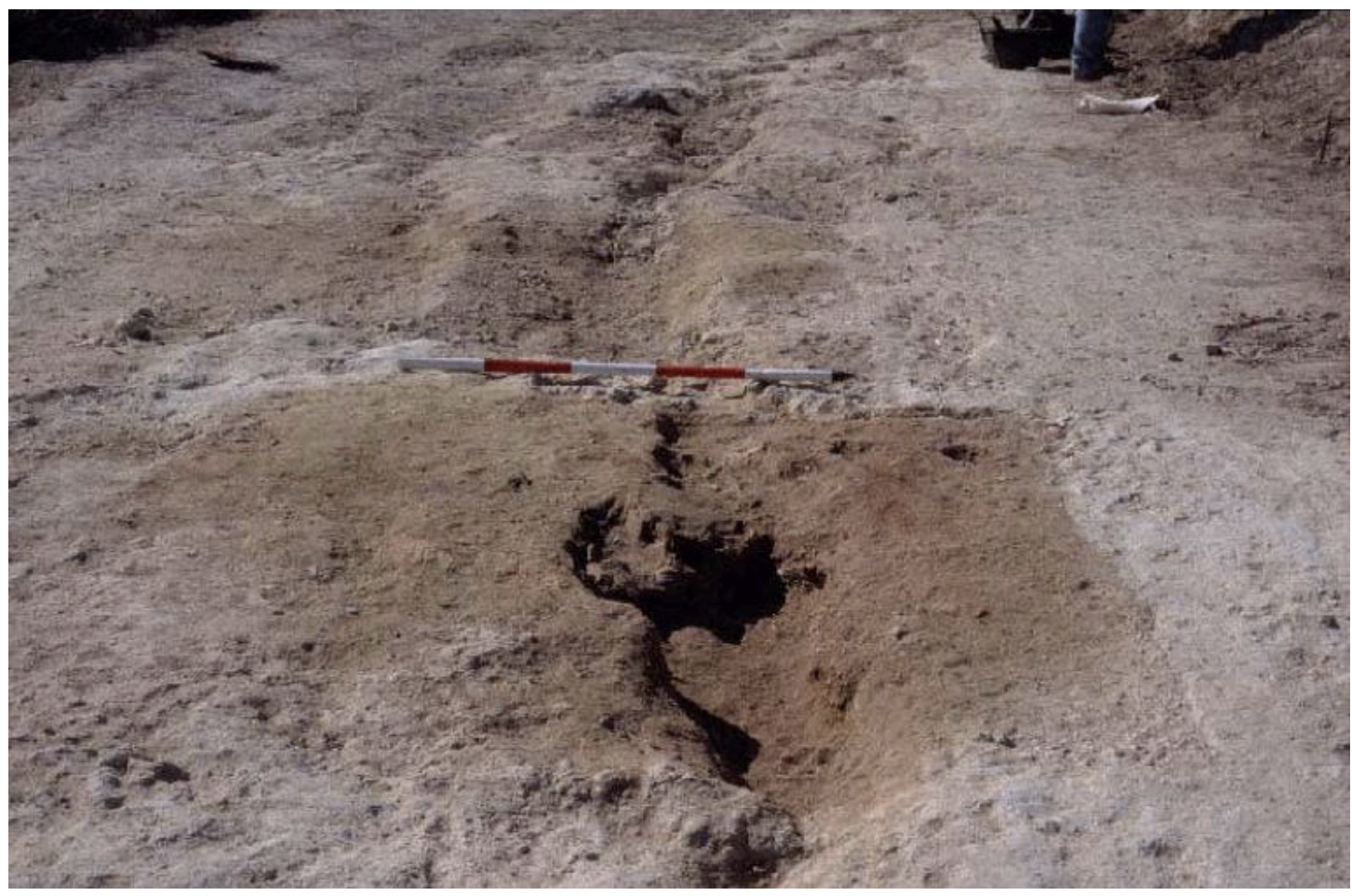

Fig.4 Close-up of track (looking south-east), after cleaning, but prior to excavation. Feature 5 is in the foreground; the irregular road surface is the result of erosion.

\footnotetext{
${ }^{12}$ Pebbles were also found in a lamp in tomb A: Kythera, 228 no. 1.

${ }^{13}$ They were removed as four sherd concentrations; excavation find numbers: ${ }^{\wedge} 156, \wedge 157, \wedge 171, \wedge 173.1$ (not catalogued).
} 


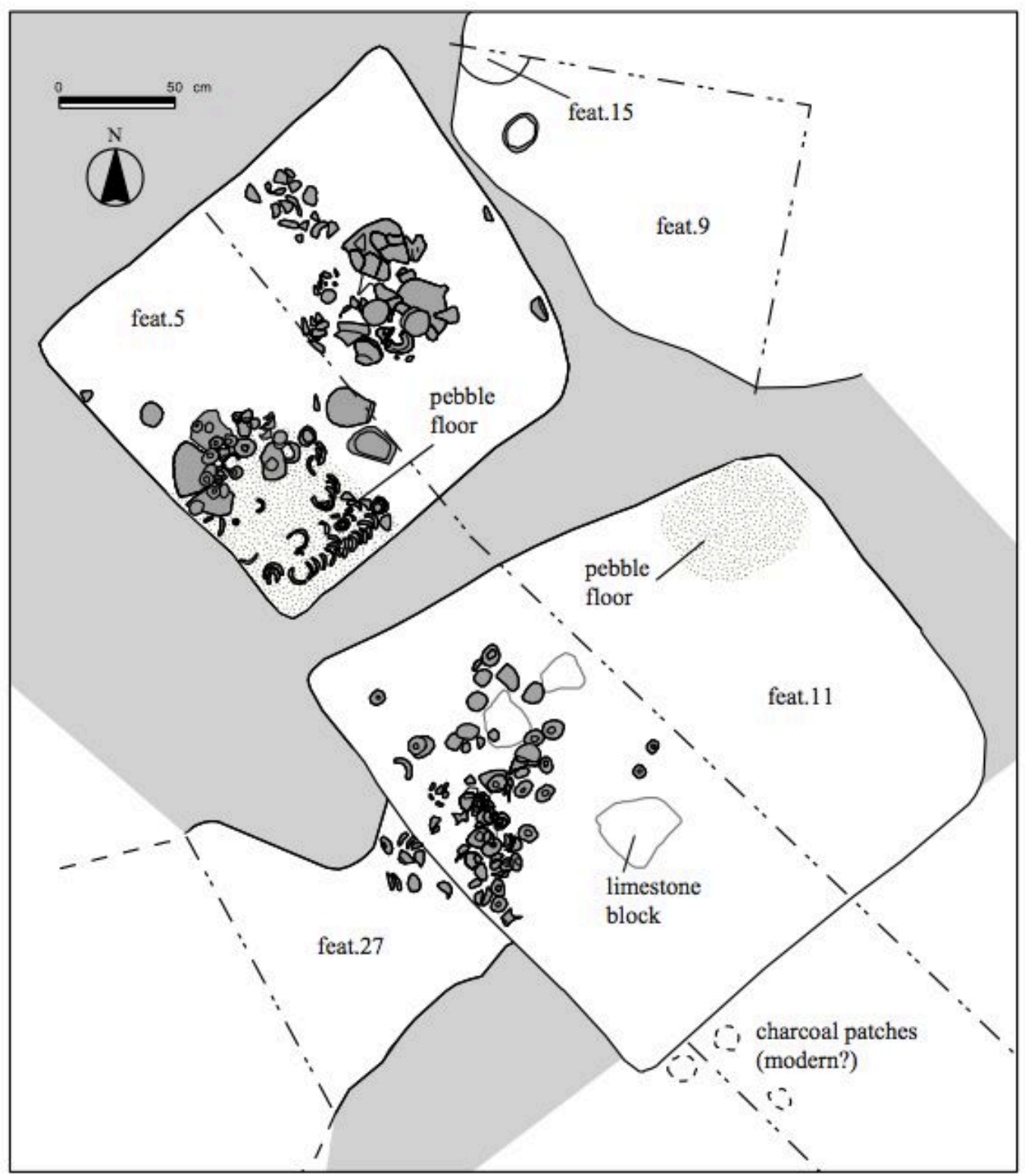

Fig.5 Features 5, 9, 11 and 27 with drawn pottery scatters and pebbled areas. 


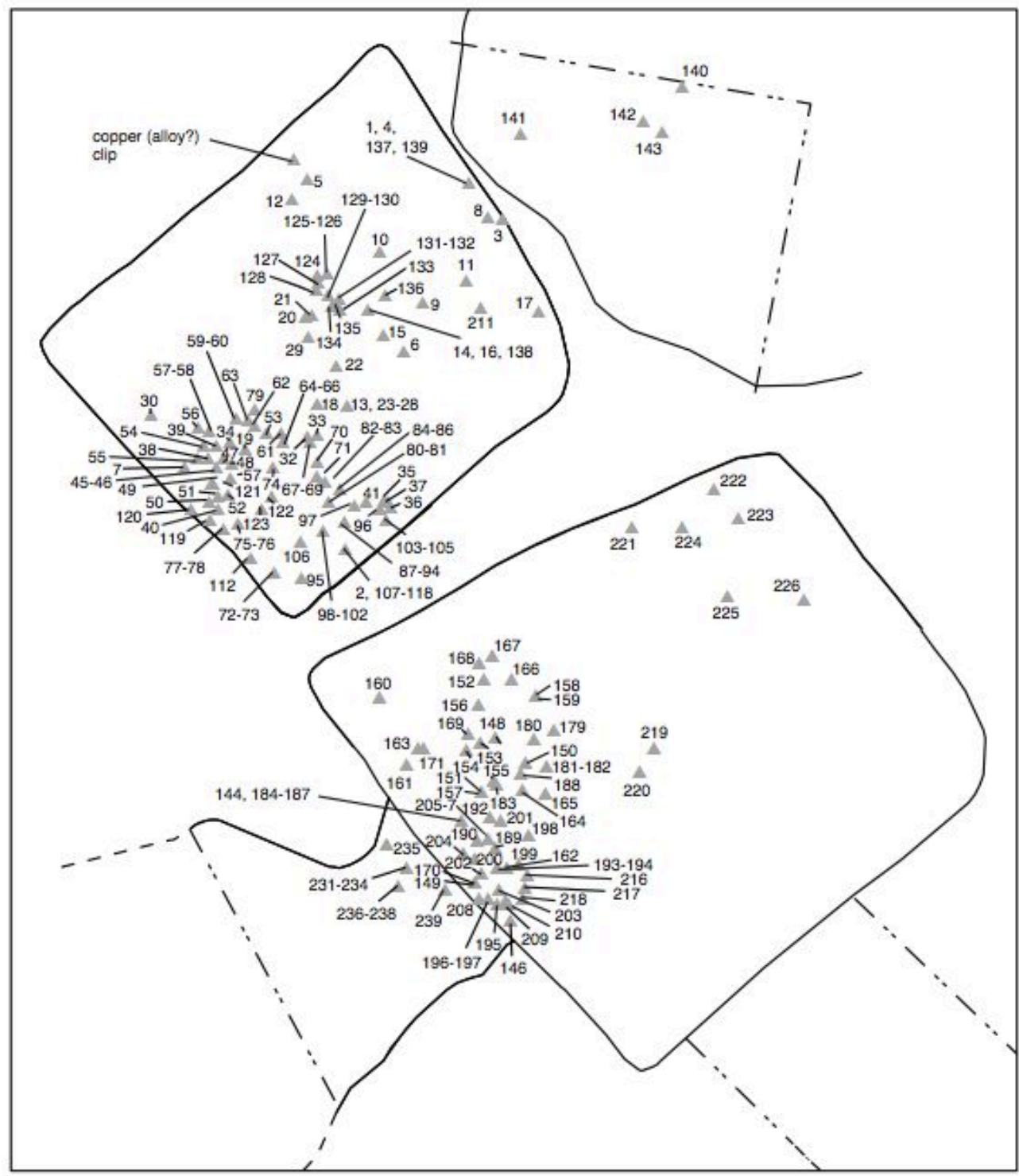

Fig.6 Features 5, 9, 11 and 27 with location of catalogued finds.

\section{Feature 9 (FIGS. 5-6)}

Feature 9 was at first hard to define as its single fill clearly incorporated some redeposited bedrock inclusions. It appears to be the bottom of an irregular feature but, as only a part was excavated, is of unknown extent. The lower parts of two closed vessels were found, probably jugs $(140,141)$, one with dense perforations on the shoulder, along with the scattered remnants of a few conical cups (two reconstructable ones, 142-143, and base fragments of at least three more). A small apparently circular feature (15) possessing near vertical sides, a flattish base and a single mid brown silty clay fill was overlain by the primary fill of feature 9.

Feature 11 (FIGS. 4-6) 
Feature 11 ( 225 x $225 \mathrm{~cm}$, max. depth $14 \mathrm{~cm}$ ) was generally well-defined, but with an eastern edge that petered out with the descending terrain. It was roughly square in plan with a generally flat base (except for an irregular depression where the section was drawn) and near vertical sides, cut slightly into the slope. The main infilling episode of a reddish-brown silty clay is similar to the one found in feature 5, but also includes a few limestone blocks.

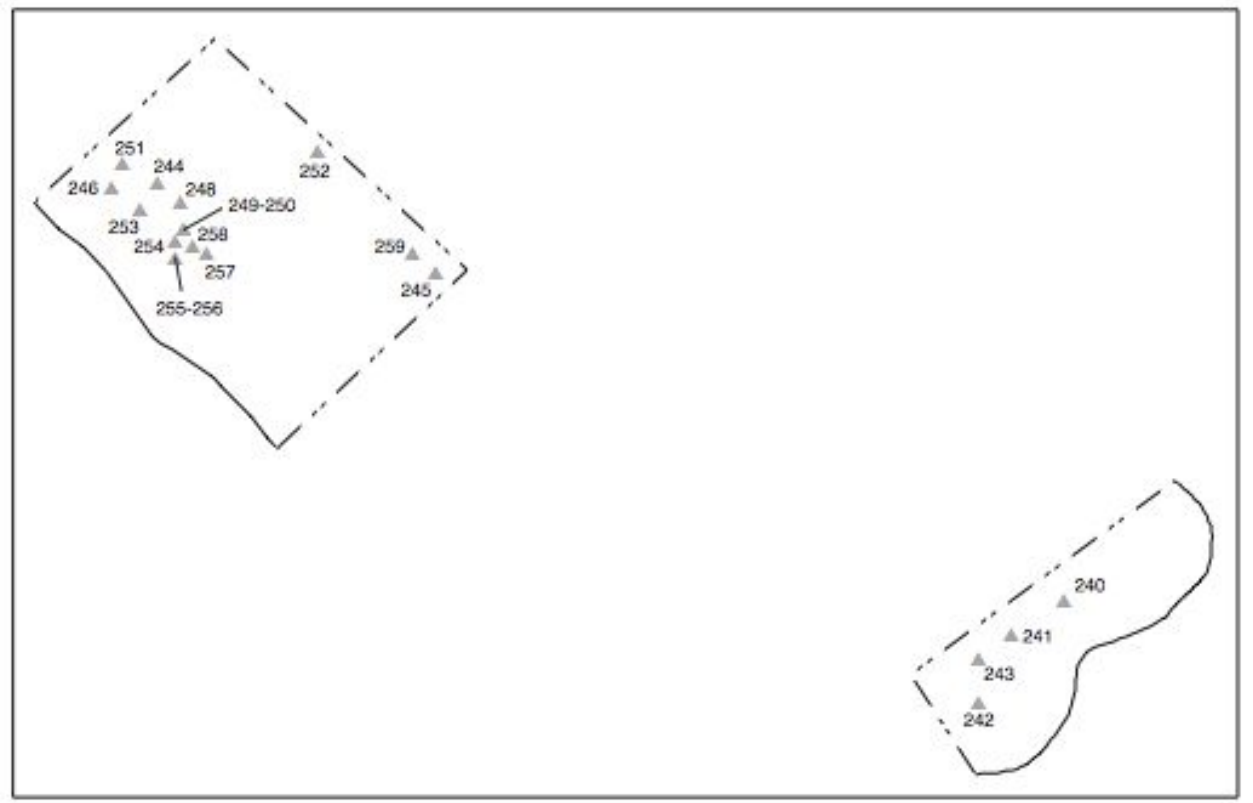

Fig.7 Features 21 and 25 with drawn pottery scatters and pebbled area.

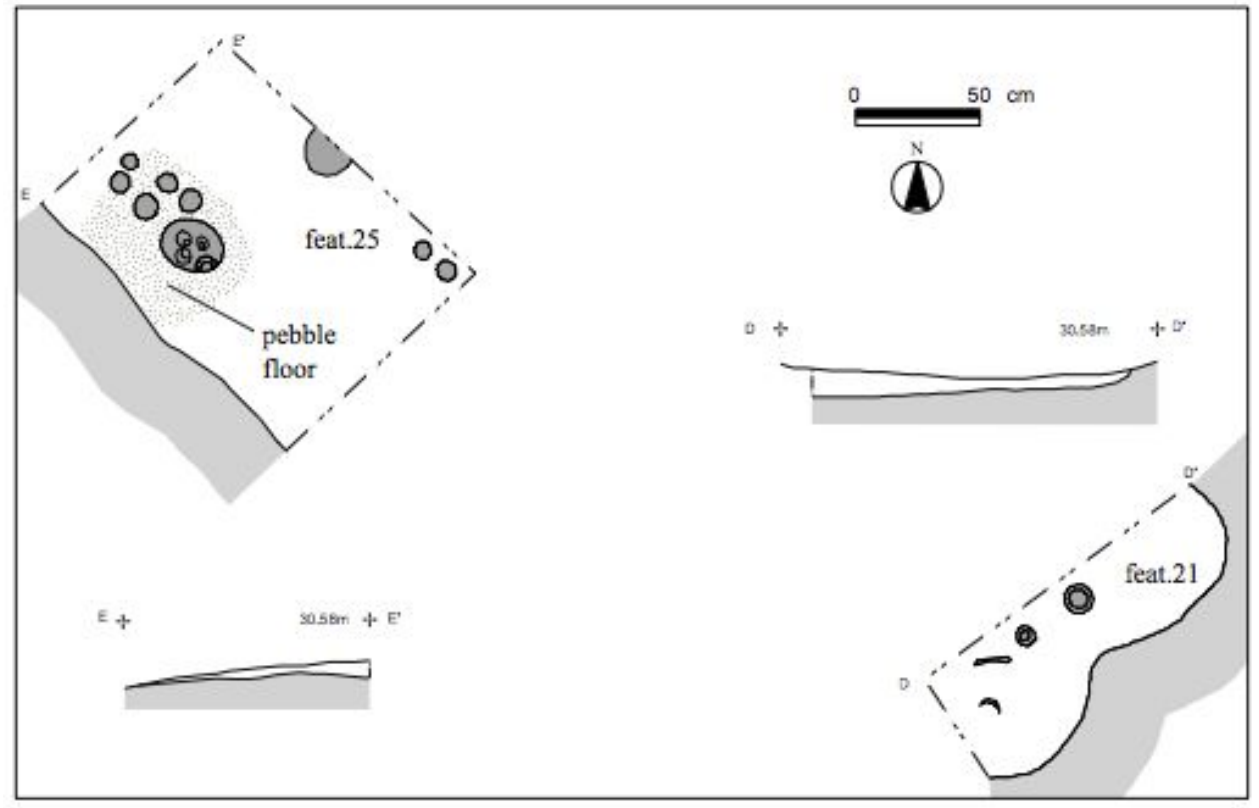

Fig.8 Features 21 and 25 with location of catalogued finds. 
Also similar to feature 5 is a localised layer of pebbles under some conical cup fragments (221-226), this time in the north-west corner. Apart from these fragments, the majority of the pottery was found scattered on the south-western side of the feature, along with a few irregular limestone blocks. The vast majority of the recovered pottery comprised conical cups. Out of 87 catalogued vessels, 79 were conical cups, all of the same thin-walled type (both those from the pebbled area and from the scatter). As is the case with feature 5, types other than the conical cup only occur in ones or twos: stemmed cup (1), tumbler (1), kalathoi (2), ring-handled basin (1), conical bowl (1), blossom bowl (1) and a ewer (1). Among the uncatalogued sherds recovered from the fill of the feature (c. 900 mostly tiny pieces), there are fragments of at least four hemispherical cups, one of which had dark-on-light spirals, and one possible Vapheio cup. The majority of the uncatalogued sherds comprises degraded conical cups (base fragments of at least 22 more conical cups of the same thin-walled type were noted).

\section{Feature 27 (FIGS. 5-6)}

Feature 27 was only partially excavated, but is irregular in plan and extends to the south of 11. Its single light brown, silty clay fill includes mainly conical cup fragments. Apart from the nine catalogued conical cups, c. 70 more fragments of degraded ones were recovered; they were all of the same thin-walled type as those from feature 11, along with c. 20 small sherds from a red-slipped kalathos. Arguably, the same fill slumps into feature 11 as well. The relationship between these two features is discussed in further detail below.

Features 21 and 25 (FIGS. 7-8, PLATE 2d)

Like features 9 and 27, these were only partially investigated in small slots where pottery was visible on the surface. Feature 25 looks as if it may have been similar in its assemblage to 5 and 11. Like these, it produced conical cups placed on a small patch of pebbles. As in feature 5 , some of the cups were inverted in the middle of a conical bowl (250). A total of 16 catalogued pots came from this feature, of which six were conical cups. The rest included vessel types similar to those of features 5 and 11: hemispherical cup (1), stemmed cup (1), tumblers (2), kalathoi (2), conical bowl (1), small blossom bowl (1) and other closed vessels, probably jugs or ewers (2). Moreover, among the uncatalogued pottery, there were fragments of at least two or three more conical cups and possibly of another brown-slipped kalathos.

Feature 21 appears to have been irregular in plan. There are four catalogued vessels from this feature, all fragmentary. Most seem to be of types similar to those found in the other features: two closed vessels, possibly a ewer and a lowspouted jug, a kalathos, but also a small, narrow-necked vessel with a globular upper body (most probably a rhyton, or perhaps an alabastron?).

\section{Other Features}

In the small slot made downhill from feature 11 (FIG. 2) several other deposits were discovered but not fully excavated. Deposits 28 and 32 were of light brown 
silty clay with a few small inclusions of marl limestone. Both seem to be slumps (from the north-west and south-east) into an irregular cavity. A total of c. 130 abraded sherds were recovered from these deposits (c. 100 from 28 and c. 30 from 32). Apart from conical cup fragments, this pottery includes types of Early Bronze Age date (see below, Comment on fabrics) and a possible Mycenaean kylix sherd. On top of these features was found a series of irregular stones (feature 33), some of which are inconsistent with the local bedrock (i.e. are not marl limestone) and appear to have been deliberately placed there, perhaps to provide purchase for those travelling along the track. Deposit 19 was excavated in the area south-east of a line of bedrock limestone stretching across the site. This line of bedrock probably points to the existence of a small limestone scarp in this area that has since been eroded and/or filled in (deposit 19?) to form the existing trackway. The pottery from deposit 19 (c. 35 sherds) appears quite mixed in date, with fabrics dating from the Early Bronze Age to the Neopalatial period (see below, Comment on fabrics), as well as three possible plain Archaic/Classical sherds. These last fragments are the only non-prehistoric sherds encountered on the excavation.

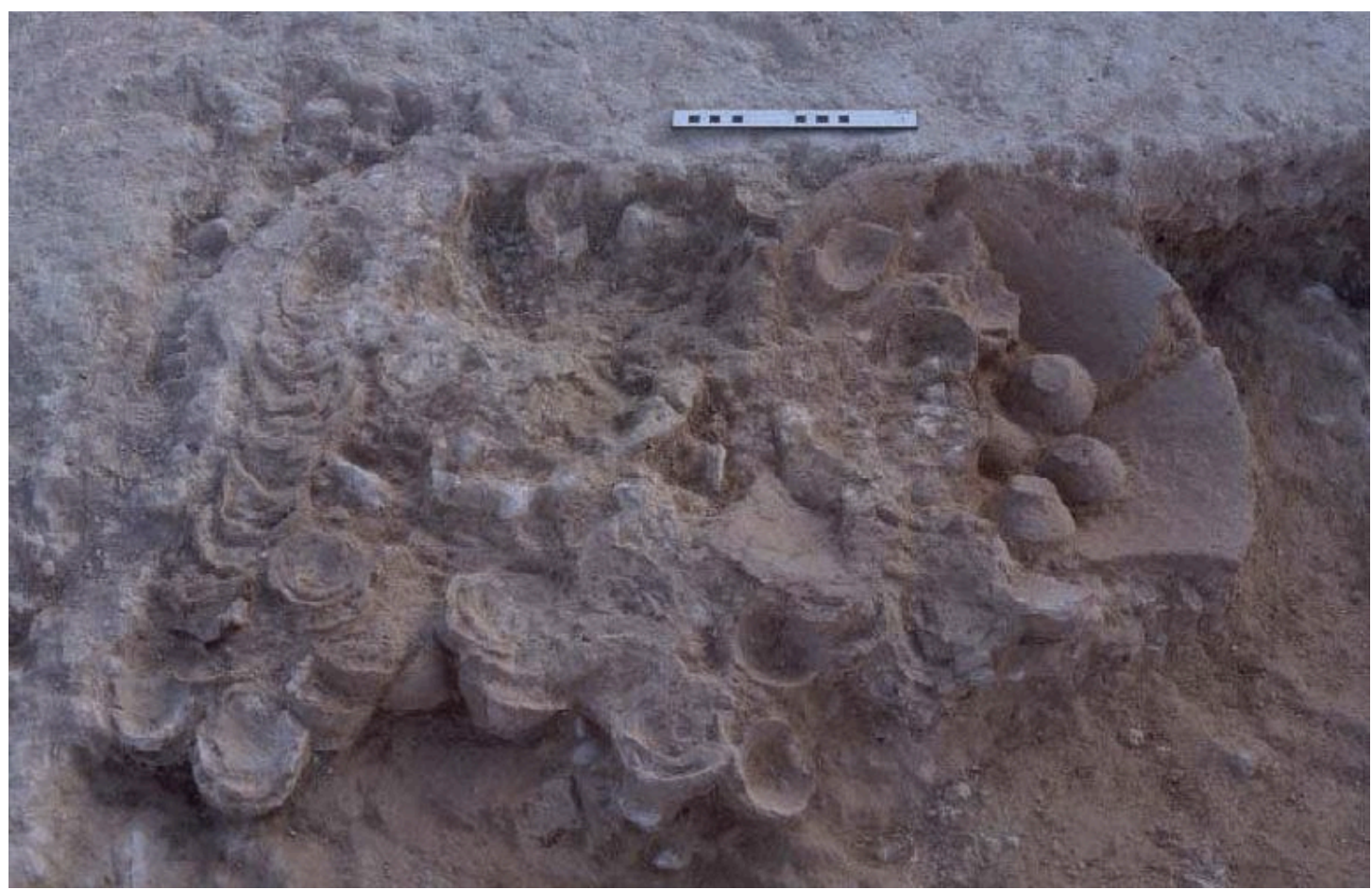

Fig.9 Close-up of south-east corner of feature 5 with conical cups stacked or inverted in a conical bowl. Pebbled area is underneath but not visible here.

\section{Pottery Catalogue}

The following catalogue lists 259 whole or largely restorable vessels grouped by feature. It should be noted that sherd material suggests that the original number of vessels may have been somewhat higher.

\section{Feature 5}


$1\left(177.2^{*}\right) \quad$ cup, hemispherical or stemmed, everted rim and thin vertical strap handle, quite fragmentary, only upper part preserved, fine pale buff, wheelmade, smoothed surfaces, dark-on-light decoration of two bands on interior rim, otherwise too worn to discern, Drim $13.0 \mathrm{~cm}$. (possibly same pot as 2).

2 (95.12) cup, possibly hemispherical or stemmed, with everted rim, very fragmentary, only rim sherds, medium-hard fine pale buff, wheelmade with fine rilling, plain, Drim $15 \mathrm{~cm}$, (looks very similar to 1 but found in different positions; same pot?)

3 (176) tumbler, everted ledge rim, concave walls, bulge at base, c. $60 \%$, very fragmentary, soft fine orange with grey core, wheelmade with some rilling, quite well smoothed, monochrome reddishorange slip all-over, two incised grooves just below rim, Ht $9.3 \mathrm{~cm}$, Drim $11.5 \mathrm{~cm}$, Dbase $8.2 \mathrm{~cm}$.

4 (177.3) kalathos, flat base and flaring squared rim, double horn lug at rim, 50\%, c. 40 fragments, almost complete profile, soft semi-fine pale light brown, wheel-fashioned with deep rilling, red monochrome slipped all-over, Dbase $9.0 \mathrm{~cm}$.

5 (97) ring-handled basin, with heavily everted rim, globular body, heavy flattened vertical handle with high central rib, 33\%, no base fragments, medium-soft semi-fine orange buff and mudstone tempered, wheel-fashioned with rilling, plain, deep incised notches at interior rim and exterior lip, also on edges of handle and its central rib, pres. Ht $12 \mathrm{~cm}$, Drim $25 \mathrm{~cm}$.

6 (160) ring-handled basin, low, wide and rounded, heavily everted rim (not present), large thick vertical flattened handle with raised central rib, only c. $10 \%$, fragments non-joining, semi-coarse brown and mudstone-tempered, coarser than other basins of this type, wheel-fashioned (a coil seam is visible), plain, very worn surfaces, unlike other examples handle and rim are not notched, pres. Ht $8.0 \mathrm{~cm}$, Drim $26 \mathrm{~cm}$.

7 (67) conical bowl, plain rim, flaring body, lug? at mid body, flat base, $66 \%, 70-80$ fragments, almost complete profile, coarse light reddish-brown and mudstone-tempered, light grey core, coilbuilt, plain, base is quite worn, $\mathrm{Ht}$ pres. $13.3 \mathrm{~cm}$, Drim $39 \mathrm{~cm}$.

8 (181) blossom bowl, 66\%, c. 50 fragments, almost complete profile, semi-fine to medium-coarse pale reddish-brown and mudstonetempered, wheel-finished (with coil-joins, or two clay layers in sections), monochrome reddish-brown slip (quite worn) and plastic decoration: vertical relief imitation of lily leaves, quite thick- walled (thicker walls than 150), $\mathrm{Ht} 10-11 \mathrm{~cm}$, Drim $11 \mathrm{~cm}$, Dbase $6 \mathrm{~cm}$.

9 (151) low-spouted jug, piriform body, vertical roll handle attached just below rim, trough spout pulled out from rim; upper body almost complete $(85 \%)$, restored from c. 50 fragments, semi-coarse

\footnotetext{
${ }^{*}$ Field number (on-site recording sequence). A concordance of catalogue and field numbers, sorted by field number, is also provided at the end of this report.
} 
10 (158) low-spouted jug, piriform body, vertical roll handle attached c. $2 \mathrm{~cm}$ below rim, trough spout pulled out from rim, almost complete: restored from 30-40 fragments, medium-hard semicoarse buff, pink at core, mudstone-tempered, coil-built with horizontal paring at mid and lower body, dark-on-light spiral on either side, between spout and handle, bands at rim, base and lower body, possible band inside rim, decoration very fugitive and unclear, $\mathrm{Ht} 22.2 \mathrm{~cm}$, Drim $13.5 \mathrm{~cm}$, Dbase $10.8 \mathrm{~cm}$.

$11(149,121,2) \quad$ ewer, ovoid-conical shape with tall, narrow neck and flaring rim, 50\%, many fragments, whole profile, medium-soft semicoarse greenish buff and mudstone tempered: n.b. neck coarser than rest of vessel, wheel-fashioned (rilling), with a good deal of horizontal paring at lower mid-body, quite thin walled at Maxd, dark-on-light band at base and 2 more at lower-mid body, very fugitive thus hard to see if any more bands or spirals, $\mathrm{Ht} 32 \mathrm{~cm}$, Drim $11.5 \mathrm{~cm}$, Dbase $9.3 \mathrm{~cm}$.

12 (121) ewer, ovoid-conical body, vertical roll handle, and rim slightly pulled out where attached, $50 \%$, restored from fragments, but no rim, medium-hard semi-fine buff and mudstone-tempered, coilbuilt with horizontal paring at upper body, finger-marks and coilridge unsmoothed at shoulder interior, dark-on-light bands and spirals (roughly painted): a band at lower body, 2 at mid-body, pres. $\mathrm{Ht} 30 \mathrm{~cm}$. Dbase $11 \mathrm{~cm}$.

13 (14.3) jug/ewer, ovoid-conical body, vertical roll handle from shoulder to neck/rim(?), $70 \%$, c. 60 fragments, base/body/shoulder preserved, medium-hard medium-coarse light pinkish-brown (buff surface) and mudstone-tempered, coil-built, smoothed surface with dark-on-light black running spirals between bands, Dbase $12 \mathrm{~cm}$.

14 (172.2) globular jar, low flattened collar and 2 upturned horizontal roll handles at upper body, extremely fragmentary, 33\%, soft semicoarse light reddish-brown, mudstone-tempered, wheelfashioned with rilling, plain, effect of buff slip - firing? Drim 10.0$11.0 \mathrm{~cm}$. (goes with lid 16).

15 (161) medium-size jar, probably globular with handles on the shoulder, upturned horizontal roll handle, very fragmentary - c. 30 fragments, medium-coarse light reddish-brown, mudstonetempered, light grey core, wheelmade? with rilling, poorly preserved surface, possible traces of black paint around the base of handle. 
16 (172.1) lid, faintly convex, with roll handle, complete, 6 fragments, coarse buff mudstone tempered, hand-made, plain and unsmoothed, Drim $12.5 \mathrm{~cm}$. (it seems to go with $\mathbf{1 4}$ ).

17 (180) closed vessel, base only, c. 20 fragments, semi-coarse greenish buff and mudstone tempered, coil-built with horizontal paring, quite thick walls, surface concreted, Dbase $10.5 \mathrm{~cm}$.

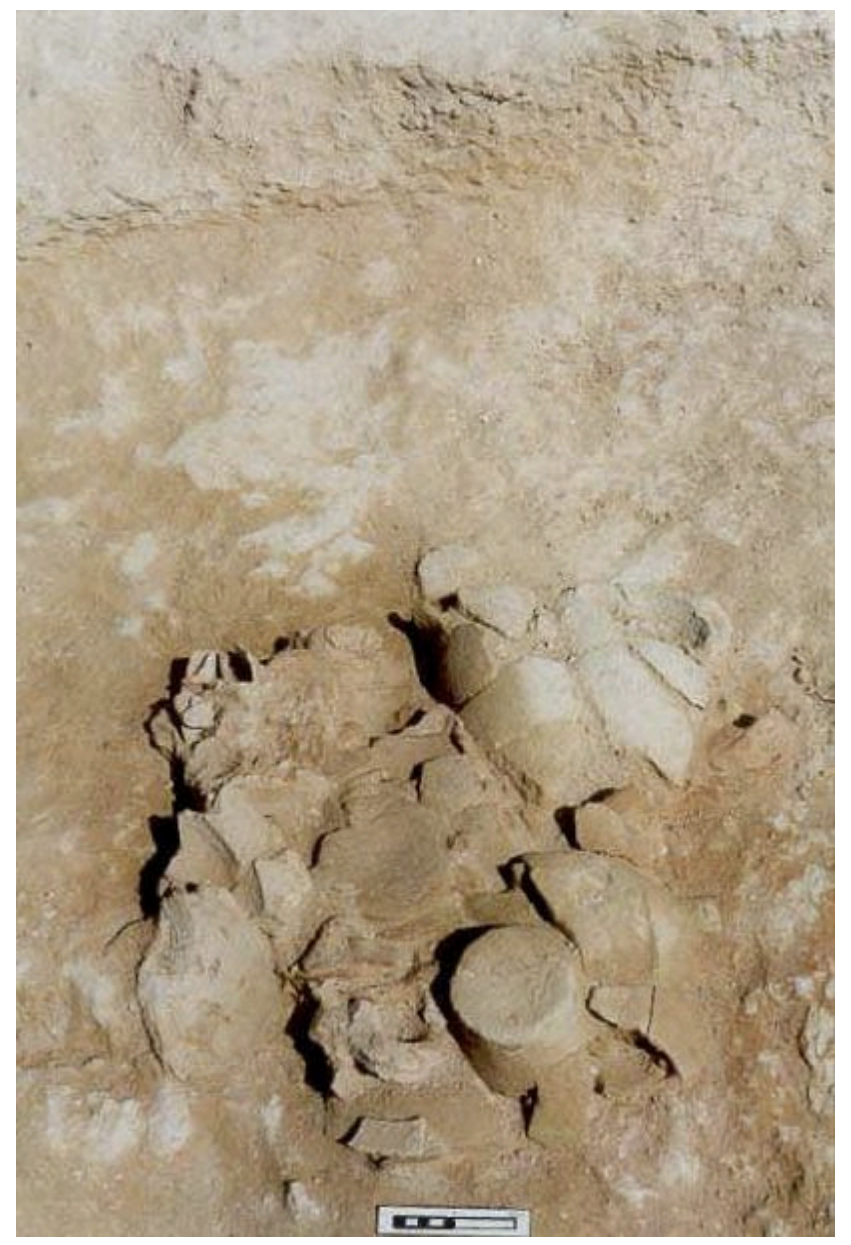

Fig.10 Close-up of centre of feature 5 with rectangular group of pottery fragments including low-spouted jug (top right).

\section{Conical cups}

All of the following are plain, thick-walled $(0.6-0.7 \mathrm{~cm})$, and wheel-fashioned (often with concentric striations at base)

18 (1) $\quad 10 \%, 2$ joining rim fragments, Drim $7.5 \mathrm{~cm}$.

19 (3) almost complete, 15 fragments, medium-soft fine pale buff, probable coil seam visible, Ht $3.9 \mathrm{~cm}$, Drim $7.9 \mathrm{~cm}$, Dbase $3.7 \mathrm{~cm}$.

20 (7) 50\%, 12 non-joining and 5 joining, soft fine brown buff, Dbase $3.6 \mathrm{~cm}$.

21 (8) 50\%, 6 joining fragments and 11 non, soft fine pale buff, Ht $3.8 \mathrm{~cm}$, Drim $8 \mathrm{~cm}$, Dbase $3.9 \mathrm{~cm}$.

22 (13) 7 rim fragments, no profile and fragmentary, semi-fine light brown with sparse mudstone inclusions. 
23 (14.1) 50\%, 12 fragments, complete profile, medium-soft semi-fine pale light brown with sparse mudstone inclusions, $\mathrm{Ht} 3.5 \mathrm{~cm}$, Drim $8.0 \mathrm{~cm}$, Dbase $3.5 \mathrm{~cm}$.

24 (14.2) 10 fragments, soft semi-fine pale pinkish-brown with sparse mudstone inclusions.

25 (14.4) almost complete, medium-soft semi-fine light brownish buff with sparse mudstone inclusions, Ht $4.1 \mathrm{~cm}$, Drim $8 \mathrm{~cm}$, Dbase $3.8 \mathrm{~cm}$.

26 (14.5) 66\%, 1 piece, medium-soft semi-fine light brownish buff with sparse mudstone inclusions, Ht $3.9 \mathrm{~cm}$, Drim $8 \mathrm{~cm}$, Dbase $3.8 \mathrm{~cm}$.

27 (14.6) $50 \%, 7$ fragments, soft semi-fine light brown with sparse mudstone inclusions, $\mathrm{Ht} 3.8 \mathrm{~cm}$, Drim $8 \mathrm{~cm}$, Dbase $3.6 \mathrm{~cm}$.

28 (14.7) 30\%, 4 fragments, medium-soft semi-fine light brownish buff with sparse mudstone inclusions, $\mathrm{Ht} 3.8 \mathrm{~cm}$, Drim $8.1 \mathrm{~cm}$, Dbase $3.7 \mathrm{~cm}$.

29 (17) complete, 12 fragments, fine to semi-fine pale brown buff, Ht 3.5$4.0 \mathrm{~cm}$, Drim $8.5 \mathrm{~cm}$, Dbase $3.7 \mathrm{~cm}$.

30 (18) almost complete, 6 fragments, fine to semi-fine pale buff with sparse mudstones, traces of coil seams visible, Ht $4.0 \mathrm{~cm}$, Drim $8.0 \mathrm{~cm}$, Dbase $4.3 \mathrm{~cm}$.

31 (22) 66\%, 10 fragments, soft fine buff (orange at core), Ht 3.6cm, Drim $8.1 \mathrm{~cm}$, Dbase $3.6 \mathrm{~cm}$.

32 (34) complete, 4 fragments, medium-soft fine pale buff with sparse mudstone inclusions, hints of coil seams close to rim, Ht 3.6$4.2 \mathrm{~cm}$, Drim $7.9 \mathrm{~cm}$, Dbase $4.1 \mathrm{~cm}$.

33 (35) $\quad 80 \%, 7$ fragments, medium-soft fine pale buff, traces of coil seams visible, Ht $3.6 \mathrm{~cm}$, Drim $8.0 \mathrm{~cm}$, Dbase $4.0 \mathrm{~cm}$.

34 (36) almost complete, 3 fragments, soft fine pale brown buff, clear traces of coil seams, Ht 3.2-4.1cm, Drim $8.3 \mathrm{~cm}$, Dbase $3.7 \mathrm{~cm}$.

35 (38) complete, 5 fragments, fine pale orange buff, traces of coil seams visible, Ht $3.9 \mathrm{~cm}$, Drim $7.9 \mathrm{~cm}$, Dbase $3.4 \mathrm{~cm}$.

36 (39) $50 \%, 6$ fragments, fine buff, traces of coil seams visible, $\mathrm{Ht} 4.0 \mathrm{~cm}$, Drim $8.0 \mathrm{~cm}$, Dbase $4.1 \mathrm{~cm}$.

$37(40)$

$38(41)$

$33 \%$, 8 fragments, soft fine pale orange buff, Dbase $4.0 \mathrm{~cm}$.

complete, 7 fragments, medium-hard fine pale buff, very clear coil seam in break, Ht $3.8 \mathrm{~cm}$, Drim $8.3 \mathrm{~cm}$, Dbase $3.5 \mathrm{~cm}$.

39 (42) complete, 10 fragments, soft fine pale-brown buff with sparse mudstone inclusions, $\mathrm{Ht} 3.9 \mathrm{~cm}$, Drim $7.7 \mathrm{~cm}$, Dbase $3.3 \mathrm{~cm}$.

40 (43) $\quad 50 \%, 7$ fragments, fine to semi-fine pale buff, $\mathrm{Ht} 3.4 \mathrm{~cm}$, Drim $8.0 \mathrm{~cm}$, Dbase $4.4 \mathrm{~cm}$.

41 (44) almost complete, 8 fragments, medium-soft fine orange, clear coil seams at break, Ht $4.0 \mathrm{~cm}$, Drim $7.7 \mathrm{~cm}$, Dbase $3.5 \mathrm{~cm}$.

42 (49) 15\%, very fragmentary ( $>30$ sherds), medium-soft fine orangebrown.

43 (50) complete, 12 fragments, soft semi-fine pale brown/buff with sparse mudstone inclusions, irregular wobbly base, $\mathrm{Ht} 4.1 \mathrm{~cm}$, Drim $8.0 \mathrm{~cm}$, Dbase $3.4 \mathrm{~cm}$.

44 (54) $66 \%$, at least 7 fragments, soft fine brown/buff, Ht 3.8cm, Drim $8.0 \mathrm{~cm}$, Dbase $3.7 \mathrm{~cm}$. 
45 (57.1) complete, 2 fragments ( 2 halves), soft fine pale brown/buff, extremely clear coil seams (at least 3), $\mathrm{Ht} 3,5 \mathrm{~cm}$, Drim $8.1 \mathrm{~cm}$, Dbase $4.0 \mathrm{~cm}$.

46 (57.2) complete, 4 fragments, medium hard fine pale buff with large lime spall, faint traces of coils, Ht $3.3-3.9 \mathrm{~cm}$, Drim $8.3 \mathrm{~cm}$, Dbase $3.8 \mathrm{~cm}$.

47 (58) complete, 6 fragments, medium-hard fine pale buff, really well smoothed, Ht $3.5 \mathrm{~cm}$, Drim $8.1 \mathrm{~cm}$, Dbase $3.7 \mathrm{~cm}$.

48 (59) complete, 3 fragments, medium-soft semi-fine buff to orange, very clear example of coil seam in break, $H t 3.5 \mathrm{~cm}$, Drim $8.0 \mathrm{~cm}$, Dbase $4.1 \mathrm{~cm}$.

49 (60) almost complete, 10 fragments, medium-soft fine pale buff, Ht $4.0 \mathrm{~cm}$, Drim $8.1 \mathrm{~cm}$, Dbase $3.7 \mathrm{~cm}$.

50 (61) almost complete, 9 fragments, soft semi-fine brown/buff, Ht $3.3 \mathrm{~cm}$, Drim $7.6 \mathrm{~cm}$, Dbase $3.8 \mathrm{~cm}$.

51 (62) almost complete, 1 fragment, medium-hard fine pale buff, Ht $3.9 \mathrm{~cm}$, Drim $8.3 \mathrm{~cm}$, Dbase $3.7 \mathrm{~cm}$.

52 (63) $12 \%$, rim only, 8 fragments, some joining, soft semi-fine pale brown/buff, Drim $7.5 \mathrm{~cm}$.

53 (64) very fragmentary; 17 non-joining fragments, semi- fine brown with sparse mudstone inclusions, plain.

54 (65) almost complete, 5 fragments, medium-hard fine pale buff, Ht 3.4- $4.2 \mathrm{~cm}$, Drim $8.2 \mathrm{~cm}$, Dbase $3.6 \mathrm{~cm}$.

55 (66) almost complete, 12 fragments, soft semi-fine pale brown/buff with a few brown mudstone inclusions, traces of coil seams, $\mathrm{Ht}$ 3.5- 3.8cm; Drim 7.4- $8.2 \mathrm{~cm}$, Dbase $3.7 \mathrm{~cm}$.

56 (68) $80 \%, 11$ fragments, soft fine orange, $\mathrm{Ht} 4.0 \mathrm{~cm}$, Drim $7.6 \mathrm{~cm}$, Dbase $3.7 \mathrm{~cm}$.

57 (71.1) complete, 5 fragments, medium hard fine pale buff, $\mathrm{Ht} 3.8 \mathrm{~cm}$, Dbase $3.7 \mathrm{~cm}$.

58 (71.2) almost complete, 9 fragments, medium soft fine pale brown/buff, Ht $3.9 \mathrm{~cm}$, Drim $8.2 \mathrm{~cm}$, Dbase $3.3 \mathrm{~cm}$.

59 (72.1) complete, 6 fragments, medium hard, fine pale buff with sparse mudstone inclusions, traces of coil seams, irregular rim, $\mathrm{Ht}$ $3.5 \mathrm{~cm}-3.9 \mathrm{~cm}$, Drim $7.9 \mathrm{~cm}-8.6 \mathrm{~cm}$, Dbase $3.6 \mathrm{~cm}$.

60 (72.2) almost complete, 1 piece, medium-hard fine pale buff with brown mudstone inclusions, hints of coil seams, Drim $8.0 \mathrm{~cm}$, Dbase $3.1 \mathrm{~cm}$.

$61(73) \quad 50 \%, 9$ fragments, soft semi- fine brown/buff, Ht $3.3 \mathrm{~cm}$, Drim $7.5 \mathrm{~cm}$, Dbase $3.6 \mathrm{~cm}$.

$62(74) \quad 80 \%, 11$ fragments, medium hard fine orange-buff, Ht $3.6 \mathrm{~cm}$, Drim $7.6 \mathrm{~cm}$, Dbase $3.3 \mathrm{~cm}$.

63 (75) 75\%, 10 fragments, medium soft fine pale buff, Ht $4.2 \mathrm{~cm}$, Drim $8.6 \mathrm{~cm}$, Dbase $4.0 \mathrm{~cm}$.

64 (76.1) 66\%, 7 fragments and others non- joining, soft fine brown/ buff, traces of coil seams, Ht $3.8 \mathrm{~cm}$, Drim $7.5 \mathrm{~cm}$, Dbase $3.5 \mathrm{~cm}$.

65 (76.2) 33\%, 11 fragments, medium hard fine pale buff, traces of coil seams, Drim $7.5 \mathrm{~cm}$.

66 (76.3) 33\%, extremely fragmentary, soft semi- fine brown. 
67 (77.1) 75\%, 5 fragments, fine pale buff, medium hard, $\mathrm{Ht} 3.7 \mathrm{~cm}$, Drim $8.5 \mathrm{~cm}$, Dbase $4.1 \mathrm{~cm}$.

$68(77.2) \quad 50 \%, 6$ fragments, medium soft fine pale buff, Ht $3.8 \mathrm{~cm}$, Drim $7.5 \mathrm{~cm}$, Dbase $3.6 \mathrm{~cm}$.

69 (77.3) 50\%, 17 joining and 1 non-joining fragments, soft semi- fine brown, Dbase $3.5 \mathrm{~cm}$.

70 (78) 75\%; 5 fragments, medium hard fine pale buff with sparse mudstone inclusions, $\mathrm{Ht} 3.5 \mathrm{~cm}$, Dbase $4.2 \mathrm{~cm}$.

71 (79) 12\%, 4 joining rim sherds, medium soft fine pale buff, traces of coil seams, Ht $8.0 \mathrm{~cm}$.

72 (80.1) 75\%, 11 fragments, medium soft fine pale buff with very sparse mudstone inclusions, $\mathrm{Ht} 3.8 \mathrm{~cm}$, Drim $7.8 \mathrm{~cm}$, Dbase $3.5 \mathrm{~cm}$.

73 (80.2) 50\%, 7 joining and c. 20 non- joining fragments, medium soft semi- fine brown/buff, Ht $3.4 \mathrm{~cm}$.

74 (81) $\quad 10 \%, 7$ very small joining fragments of base, medium hard fine pale buff with pinkish core.

75 (82.1) complete, 1 piece, medium hard fine pale buff, faint traces of coil seams, Ht $4.1 \mathrm{~cm}$, Drim $8.5 \mathrm{~cm}$, Dbase $4.2 \mathrm{~cm}$.

76 (82.2) $\quad 80 \%, 7$ fragments, medium-soft fine orange/buff with pinkish core, traces of coil seams, Ht $3.3 \mathrm{~cm}$, Drim $8.0 \mathrm{~cm}$, Dbase $3.6 \mathrm{~cm}$.

77 (83.1) 33\%, 6 fragments, medium hard fine pale buff, Dbase $3.7 \mathrm{~cm}$.

78 (83.2) 75\%, 4 fragments, medium hard fine pale buff, Ht $3.2 \mathrm{~cm}$, Drim $8.6 \mathrm{~cm}$, Dbase $3.7 \mathrm{~cm}$.

79 (84) very fragmentary, c. 35 fragments, medium soft semi- fine pale light brown.

80 (85.1) $\quad 50 \%, 4$ fragments, medium hard fine pale buff, $\mathrm{Ht} 3.7 \mathrm{~cm}$, Drim $8.1 \mathrm{~cm}$, Dbase $3.7 \mathrm{~cm}$.

81 (85.2) 50\%, 15 joining and c. 20 non-joining fragments, soft semi- fine brown/buff, Ht $3.8 \mathrm{~cm}$, Drim $8.0 \mathrm{~cm}$, Dbase $4.1 \mathrm{~cm}$.

82 (86.1) 66\%, c. 25 fragments, medium soft semi-fine pale light brown with sparse mudstone inclusions, Ht $3.6 \mathrm{~cm}$, Drim $8.0 \mathrm{~cm}$, Dbase $4.0 \mathrm{~cm}$.

83 (86.2) very fragmentary, soft semi-fine reddish-brown, Ht $3.6 \mathrm{~cm}$, Dbase $3.5-4.0 \mathrm{~cm}$.

84 (87.1) complete, 7 fragments, fine pale buff, really well-smoothed, Drim $8.5 \mathrm{~cm}$, Dbase $3.8 \mathrm{~cm}$.

85 (87.2) 50\%, 11 fragments, medium hard fine pale pinkish buff, Ht $3.8 \mathrm{~cm}$, Drim $8.0 \mathrm{~cm}$, Dbase $3.6 \mathrm{~cm}$.

86 (87.3) $\quad 80 \%, 45$ fragments, soft fine pale brown/buff, Ht $3.0 \mathrm{~cm}$, Drim $8.4 \mathrm{~cm}$, Dbase $3.8 \mathrm{~cm}$.

87 (88) 33\%, 1 piece and many small non-joining fragments, mediumhard fine buff with sparse mudstone inclusions, coil seam very clear in break, Ht $4.0 \mathrm{~cm}$, Drim $8.0 \mathrm{~cm}$, Dbase $3.6 \mathrm{~cm}$.

88 (88.1) complete, 6 fragments, soft semi-fine pale brown/buff, $\mathrm{Ht} 3.5 \mathrm{~cm}$, Drim $8.0 \mathrm{~cm}$, Dbase $3.8 \mathrm{~cm}$.

89 (88.2) almost complete, 6 fragments, soft fine buff, Ht $3.8 \mathrm{~cm}$, Drim $8.1 \mathrm{~cm}$, Dbase $3.3 \mathrm{~cm}$.

90 (88.3) complete, 5 fragments, soft fine pale buff, very clear coil seams, $\mathrm{Ht} 3.8 \mathrm{~cm}$, Drim $8.5 \mathrm{~cm}$, Dbase $4.2 \mathrm{~cm}$. 
91 (88.4) 50\%, 3 joining fragments and other very small ones, medium hard fine pale buff, large lump under base (irregular), $\mathrm{Ht} 4.0 \mathrm{~cm}$, Drim $7.0 \mathrm{~cm}$, Dbase $3.5 \mathrm{~cm}$.

92 (88.5) almost complete, 13 fragments, medium hard fine buff, $\mathrm{Ht} 3.5 \mathrm{~cm}$, Drim $8.0 \mathrm{~cm}$, Dbase $3.5 \mathrm{~cm}$.

93 (88.6) 50\%, 5 fragments, medium soft semi-fine pale buff with sparse mudstone inclusions, $\mathrm{Ht} 3.5 \mathrm{~cm}$, Drim $8.0 \mathrm{~cm}$.

94 (88.7) 50\%, 10 fragments, soft fine buff, Ht $3.6 \mathrm{~cm}$, Drim $8.0 \mathrm{~cm}$, Dbase $3.6 \mathrm{~cm}$.

95 (89) 25\%, 3 joining and c. 20 non-joining fragments, medium hard semi-fine brown/buff.

96 (90) almost 50\%; 9 fragments, medium hard fine pale brown/buff, coil seams visible in break, Ht $3.9 \mathrm{~cm}$, Drim $8.0 \mathrm{~cm}$, Dbase $3.9 \mathrm{~cm}$.

97 (91) 25\%, 3 fragments, medium hard fine pale buff, coil seams visible, Ht $3.2 \mathrm{~cm}$, Drim $8.0 \mathrm{~cm}$.

98 (92.1) almost complete, 12 fragments, medium soft fine pale buff, Ht $4.0 \mathrm{~cm}$, Drim $8.5 \mathrm{~cm}$, Dbase $3.5 \mathrm{~cm}$.

99 (92.2) 50\%, 11 fragments, soft fine pale buff, Ht $3.3 \mathrm{~cm}$, Drim $8.0 \mathrm{~cm}$, Dbase $3.7 \mathrm{~cm}$.

100 (92.3) 33\%, 1 piece, medium hard fine pale buff, coil seams in break, Ht $3.4 \mathrm{~cm}$, Drim $7.5 \mathrm{~cm}$, Dbase $3.2 \mathrm{~cm}$.

$101(92.4) \quad 50 \%, 9$ fragments and many tiny pieces, soft fine pale brown/buff, Ht $3.7 \mathrm{~cm}$, Drim $8.0 \mathrm{~cm}$, Dbase $3.8 \mathrm{~cm}$.

102 (92.5) 50\%, 13 fragments, medium hard semi- fine pale light brown with very sparse mudstone inclusions, coil seams visible in break, Ht $3.8 \mathrm{~cm}$, Drim $8.5 \mathrm{~cm}$, Dbase $3.6 \mathrm{~cm}$.

103 (93.1) 70\%, 6 fragments, fine pale brown buff, Ht $3.8 \mathrm{~cm}$, Drim $7.8 \mathrm{~cm}$, Dbase $3.6 \mathrm{~cm}$.

$104(93.2) \quad 50 \%, 12$ fragments, fine buff, Ht $3.9 \mathrm{~cm}$, Drim $8.0 \mathrm{~cm}$, Dbase $3.9 \mathrm{~cm}$.

105 (93.3) 25\%, c. 10 fragments, fine buff, pinkish orange core, Ht $4.0 \mathrm{~cm}$, Drim $8.0 \mathrm{~cm}$, Dbase $3.6 \mathrm{~cm}$.

106 (94) 75\%, c. 20 fragments, fine buff, pinkish at core, $\mathrm{Ht} 3.7 \mathrm{~cm}$, Drim $7.7 \mathrm{~cm}$, Dbase $3.6 \mathrm{~cm}$.

107 (95.1) almost complete, 8 fragments, medium soft semi-fine pale light brown, Ht $3.1 \mathrm{~cm}$, Drim $8.5 \mathrm{~cm}$, Dbase $3.6 \mathrm{~cm}$.

$108(95.2) \quad 50 \%$, c. 40 fragments, medium hard semi-fine light brown, Ht $3.4 \mathrm{~cm}$, Drim $8.4 \mathrm{~cm}$, Dbase $4.0 \mathrm{~cm}$.

109 (95.3) 40\%, c. 20 fragments, medium soft semi-fine pale light brown with sparse mudstone inclusions, Ht $2.7 \mathrm{~cm}$, Drim $7.5 \mathrm{~cm}$, Dbase $3.4 \mathrm{~cm}$.

110 (95.4) complete, 8 fragments, medium soft fine pale brown, light red firing cloud on ext, Ht $3.6 \mathrm{~cm}$, Drim $8.5 \mathrm{~cm}$, Dbase $3.5 \mathrm{~cm}$.

111 (95.5) 33\%, 30 fragments, medium soft semi-fine light brown with sparse mudstone inclusions, Drim $9.0 \mathrm{~cm}$, Dbase $3.8 \mathrm{~cm}$.

112 (95.6) very fragmentary, 9 fragments, medium soft semi-fine pale light brown with sparse mudstone inclusions. 
113 (95.7) 25\%, 7 fragments, soft semi-fine light reddish-brown to pale brown with sparse mudstone and carbonate inclusions, Drim $8.0 \mathrm{~cm}$.

114 (95.8) 40\%, 14 fragments, medium soft semi-fine brown/buff, $\mathrm{Ht} 3.4 \mathrm{~cm}$, Drim $9.5 \mathrm{~cm}$, Dbase $3.8 \mathrm{~cm}$.

115 (95.9) 33\%, 12 fragments, soft semi-fine pale light brown with sparse mudstone inclusions, Ht $2.9 \mathrm{~cm}$, Drim $8.0 \mathrm{~cm}$, Dbase $3.6 \mathrm{~cm}$.

116 (95.10) almost complete, 7 fragments, medium soft semi-fine pale light brown with sparse mudstone inclusions, Drim $8.6 \mathrm{~cm}$, Dbase $3.8 \mathrm{~cm}$.

117 (95.11) 40\%, c. 28 fragments, medium soft semi-fine pale light brown, with light red firing clouds, Ht $2.9 \mathrm{~cm}$, Drim $10 \mathrm{~cm}$, Dbase $4.0 \mathrm{~cm}$.

118 (95.13) 66\%, 9 fragments, medium soft semi-fine pale light pinkish brown, Ht $3.0 \mathrm{~cm}$, Drim $8.4 \mathrm{~cm}$, Dbase $4.0 \mathrm{~cm}$.

$119(96) \quad 70 \%$, c. 16 fragments, fine buff, Ht $3.9 \mathrm{~cm}$, Drim $8.0 \mathrm{~cm}$, Dbase $3.9 \mathrm{~cm}$.

120 (99) 30\%, c. 21 fragments, not complete profile, semi-fine with sparse mudstone inclusions, very light grey core.

121 (130) complete, 1 piece, fine buff, Ht $3.9 \mathrm{~cm}$, Drim $8.3 \mathrm{~cm}$, Dbase $4.2 \mathrm{~cm}$.

122 (132) $\quad 30 \%$, c. 15 fragments, not complete profile, semi-fine to medium light brown to reddish-brown with rare mudstone inclusions, core locally redder than surface, Drim $8.0 \mathrm{~cm}$.

123 (133) 70\%, 4 fragments, fine buff, $\mathrm{Ht} 3.8 \mathrm{~cm}$, Drim $8.0 \mathrm{~cm}$, Dbase $3.6 \mathrm{~cm}$.

124 (148) 50\%, 5 joining fragments and 2 others, very soft semi-fine brown with spare mudstone inclusions, Ht $3.9 \mathrm{~cm}$, Drim $8.3 \mathrm{~cm}$, Dbase $3.5 \mathrm{~cm}$.

125 (152.1) 50\%, 8 fragments, very soft semi-fine brown, Ht 3.6cm, Drim $8.0 \mathrm{~cm}$, Dbase $4.2 \mathrm{~cm}$.

126 (152.2) 15\%, 2 base fragments joining and 5 non-joining, soft semi-fine brown, Dbase $3.7 \mathrm{~cm}$.

127 (153) 25\%, 6 fragments, soft semi-fine light brown, Ht 3.7-4.4cm, Drim $8.0 \mathrm{~cm}$, Dbase $3.9 \mathrm{~cm}$.

128 (154) 50\%, 10 fragments, soft fine light brown/orange, Dbase $3.5 \mathrm{~cm}$.

129 (155.1) 75\%, 6 joining and c. 20 loose fragments, medium soft semi-fine orange/ buff with sparse mudstone inclusions, coil seam in break, Ht $3.3 \mathrm{~cm}$, Drim $8.0 \mathrm{~cm}$, Dbase $3.6 \mathrm{~cm}$.

130 (155.2) complete, 3 fragments, medium hard fine pale buff, $\mathrm{Ht} 3.8 \mathrm{~cm}$, Drim $7.8 \mathrm{~cm}$, Dbase $3.7 \mathrm{~cm}$.

131 (167.1) 50\%, 4 fragments, medium hard fine buff, Ht $3.3 \mathrm{~cm}$, Drim $7.9 \mathrm{~cm}$, Dbase $3.8 \mathrm{~cm}$.

132 (167.2) 50\%, 1 piece, medium hard fine pale buff, coil seam visible in break, Ht $3.7 \mathrm{~cm}$, Drim $8.0 \mathrm{~cm}$, Dbase $3.5 \mathrm{~cm}$.

133 (168) 75\%, 1 piece and several tiny non-joining fragments, medium hard fine pale buff, coil seam visible in break, $\mathrm{Ht} 3.6 \mathrm{~cm}$, Drim $8.4 \mathrm{~cm}$, Dbase $3.5 \mathrm{~cm}$.

134 (169) complete, 13 fragments, medium soft fine pale buff, Ht 3.5$4.0 \mathrm{~cm}$, Drim $8.0 \mathrm{~cm}$, Dbase $3.6 \mathrm{~cm}$.

135 (170) very fragmentary, medium soft semi-fine brown. 
136 (173.2) 20\%, 2 fragments, medium soft semi-fine pale light brown, Drim $8.0 \mathrm{~cm}$.

137 (177.1) 80\%, 7 fragments, medium soft semi-fine pale light brown, Ht $3.2 \mathrm{~cm}$, Drim $7.8 \mathrm{~cm}$, Dbase $3.6 \mathrm{~cm}$.

138 (172.3) 25\%, 2 fragments, medium hard semi-fine pale light brown, Ht $2.8 \mathrm{~cm}$, Drim $9.0 \mathrm{~cm}$, Dbase $4.0 \mathrm{~cm}$.

139 (177.4) 33\%, 11 joining, 8 small non-joining fragments, medium soft fine brown/buff, Dbase $3.4 \mathrm{~cm}$.

\section{Feature 9}

140 (128) closed vessel with conical lower body, probably jug, 18\% pres: base and lower body, fine pale buff with faint grey core, wheelfashioned with visible rilling internally, smoothed surface/ pared, plain, pres. Ht $13 \mathrm{~cm}$.

141 (19) jug/jar/strainer, with ovoid-conical body, 50\%, semi-coarse orange buff, mudstone-tempered, wheel-fashioned with concentric striations at base and rilling internally, plain, pierced with numerous small holes in shoulder, pres. Ht $22 \mathrm{~cm}$, Dbase $9.5 \mathrm{~cm}$.

Conical cups

142 (123)

$143(124)$

\section{Feature 11}

144 (110.1) stemmed cup with everted rim and hemispherical body, short foot with flat bottom, more than 50\% pres: c. 40 fragments and complete profile, medium-soft fine buff to light brown, wheelmade with rilling, well smoothed dark-on-light: black/ red paint (colour variation due to firing), probably a band below rim and lilies (?) in black paint, and reserved red-brown band on interior rim, see Kythera (Tomb E, find 3) for parallel, Ht $11.2 \mathrm{~cm}$, Drim $12.0 \mathrm{~cm}$, Dbase $5.0 \mathrm{~cm}$.

145 (109) tumbler, with slightly concave walls and everted ledge rim, disk base, c. $25 \%$ present (9 fragments) and whole profile, mediumsoft semi-fine buff to light brown, wheelmade?, well smoothed with some wheel ribbing lines, monochrome slipped (black) internally and externally, three incised grooves below rim, $\mathrm{Ht}$ pres. $7.5 \mathrm{~cm}$, Drim $11.0 \mathrm{~cm}$.

146 (116) kalathos with flanged base and flaring profile, squared rim, and 2 double horn lugs, c. $66 \%$ preserved, quite fragmentary, soft fine orange-buff, wheel-fashioned with rilling, lustrous brown slip internally and externally, Ht $11.0 \mathrm{~cm}$, Drim $16.0 \mathrm{~cm}$, Dbase $7.5 \mathrm{~cm}$. 
147 (15) kalathos with flaring rim and square-section lip, only rim (33\%) pres, 10 fragments, medium-hard semi-fine buff to light brown, slipped internally and externally, thinner applied brown slip to interior and thick black slip exterior, Drim $18.0 \mathrm{~cm}$.

148 (26) ring-handled basin of low broad and globular type with very everted rim (rim not present but see feature 5, find 5), 50\%, no base, 30 fragments, semi-fine buff with mudstone inclusions, wheel-fashioned with traces of a coil seam just inside rim and in the break, plain, incised notches at interior rim $\&$ also at exterior lip, pres. Ht $10 \mathrm{~cm}$, Drim $28.5 \mathrm{~cm}$.

149 (69.1) conical bowl, <10\% pres, 2 fragments, medium-soft semi-fine light brown with very few mudstone inclusions, Dbase $8.0 \mathrm{~cm}$.

150 (28) blossom bowl, with globular body, walls quite thick in lower body \& thinner in upper body, $>50 \%$ present (69 fragments), medium-soft to hard, semi-fine light reddish-brown fabric with few mudstone inclusions, grey core and wheel-finished with rilling, monochrome (black slipped) internally and externally with relief decoration of vertical grooves \& ribs imitating stone blossom bowls, grooves on exterior surface can be traced on interior surface (lower body), Drim $10 \mathrm{~cm}$, Dbase $7.5 \mathrm{~cm}$.

151 (29.1) ewer, ovoid-conical profile, 25\% present, no rim or upper body, soft semi-fine orange-buff with mudstone inclusions, varies from orange internally to pale buff externally, coil-built with horizontal paring and lumpy fingermarks, and no sign of any rilling or wheel assistance, dark-on-light bands (one at base, two at mid body) and spiral, Ht pres. $22 \mathrm{~cm}$, Dbase $11.8 \mathrm{~cm}$.

Conical cups

All of the following are plain, thin-walled $(0.3-0.4 \mathrm{~cm})$, and wheel-fashioned (often with concentric striations at base), unless otherwise stated.

$152(5)$

$153(21)$

$154(23)$

$155(25)$

$156(27)$

$157(29.2)$

$158(31.1)$

$159(31.2)$ almost complete; 12 fragments, semi-fine pale silty buff with sparse mudstone inclusions, $\mathrm{Ht} 3-4 \mathrm{~cm}$, Drim $7.2 \mathrm{~cm}$, Dbase $2.8 \mathrm{~cm}$. $15 \%$, very scrappy base fragments, soft semi-fine light brown, Dbase $3.0 \mathrm{~cm}$.

$33 \%$, c. 30 fragments, semi-fine pale-light brown, wheelmade with rilling, Ht $2.8 \mathrm{~cm}$, Dbase $2.6 \mathrm{~cm}$. $80 \%, 6$ fragments, fine buff, $\mathrm{Ht} 3.1 \mathrm{~cm}$, Drim $7.6 \mathrm{~cm}$, Dbase $3.3 \mathrm{~cm}$. $33 \%$, c. 11 fragments, no profile, semi-fine pale-reddish-brown with sparse mudstone inclusions, fragments from at least one more conical cup of the same type (of pale buff fabric), Dbase $3.0 \mathrm{~cm}$.

$25 \%, 2$ fragments, base only, semi-fine pale-light brown with rare mudstone inclusions, Dbase $3.1 \mathrm{~cm}$.

$50 \%, 25$ fragments (not all from one vessel), complete profile, semi-fine light brown-pale with rare mudstone inclusions, $3.1 \mathrm{~cm}$, $8.0 \mathrm{~cm}, 3.3 \mathrm{~cm}$.

$50 \%$, 3 fragments, complete profile, semi-fine buff-light brown, wheelmade, Ht $3.0 \mathrm{~cm}$, Drim $7.5 \mathrm{~cm}$, Dbase $3.2 \mathrm{~cm}$. 
160 (32) $50 \%$, c. 20 fragments, complete profile, semi-fine pale buff with sparse mudstone inclusions, Ht $3.4 \mathrm{~cm}$, Dbase $3.0 \mathrm{~cm}$.

161 (33) 33\%, c. 15 fragments, no profile, semi-fine pale to pink-brown, wheelmade?, Dbase $3.0 \mathrm{~cm}$.

162 (37) 33\%, no profile, medium-soft semi-fine pale pink fabric with sparse mudstones, Dbase $3.5 \mathrm{~cm}$.

163 (45) 50\%, 18 fragments, complete profile, semi-fine pale-light brown with sparse mudstone inclusions, with reddish firing cloud on interior surface, wheelmade, $\mathrm{Ht} 3.2 \mathrm{~cm}$, Dbase $2.4 \mathrm{~cm}$.

164 (46) almost complete, 11 fragments, medium-hard fine buff with sparse mudstone inclusions, Ht $3.0 \mathrm{~cm}$, Drim $7.7 \mathrm{~cm}$, Dbase $3.4 \mathrm{~cm}$.

165 (47) 50\%, 13 fragments, complete profile, semi-fine pale-light brown with sparse mudstone inclusions, wheelmade, $\mathrm{Ht} 3.1 \mathrm{~cm}$, Dbase $3.0 \mathrm{~cm}$.

166 (48) 50\%, 6 joining fragments and 13 non-joining, medium-soft fine pale buff, Ht $2.7 \mathrm{~cm}$, Drim $7.4 \mathrm{~cm}$, Dbase $3.4 \mathrm{~cm}$.

167 (51) 2 fragments and almost complete, semi-fine to medium light to reddish-brown with few mudstone and carbonate inclusions, wheelmade with rilling, light brown surfaces with reddish firing cloud, Ht $3.1 \mathrm{~cm}$, Drim $7.6 \mathrm{~cm}$, Dbase $3.0 \mathrm{~cm}$.

168 (52) 50\%, 6 fragments, complete profile, semi-fine pale-light brown, wheelmade with rilling, Ht $3.3 \mathrm{~cm}$, Drim $8.2 \mathrm{~cm}$, Dbase $3.0 \mathrm{~cm}$.

169 (53) almost complete, 3 fragments, fine pale buff, blushing orange in places, possible coil seams, $\mathrm{Ht} 3.0 \mathrm{~cm}$, Drim $7.5 \mathrm{~cm}$, Dbase $3.2 \mathrm{~cm}$.

170 (69.2) 10\%, 6 fragments, medium-soft semi-fine buff/light brown with few mudstone inclusions, wheelmade?, Dbase $3.0 \mathrm{~cm}$.

$171(70) \quad 25 \%, 10$ fragments, not complete profile, medium-soft semi-fine buff, Drim $9.0 \mathrm{~cm}$.

$172(100) \quad 66 \%, 5$ fragments, fine pale buff, $\mathrm{Ht} 3.0 \mathrm{~cm}$, Drim $6.9 \mathrm{~cm}$, Dbase $2.5 \mathrm{~cm}$.

173 (101) 75\%, 3 fragments, semi-fine pale buff, $\mathrm{Ht} 3.0 \mathrm{~cm}$, Drim $7.5 \mathrm{~cm}$, Dbase $3.0 \mathrm{~cm}$.

174 (102) 75\%, 10 fragments, complete profile, semi-fine buff to pink, wheel-made? pink interior and buff exterior surface, $H t 3.4 \mathrm{~cm}$, Drim $7.5 \mathrm{~cm}$, Dbase $3.4 \mathrm{~cm}$.

175 (103) 75\%, 7 fragments, fine pale buff, trace of coil seam, $\mathrm{Ht} 3.1 \mathrm{~cm}$, Drim $6.5 \mathrm{~cm}$, Dbase $2.9 \mathrm{~cm}$.

176 (104.1) 80\%, 17 fragments, fine buff, some orange blushing, Ht $2.6 \mathrm{~cm}$, Drim $6.7 \mathrm{~cm}$, Dbase $2.7 \mathrm{~cm}$.

177 (104.2) 66\%, 15 fragments, complete profile, medium-soft semi-fine pale light brown, wheelmade with rilling, Ht $2.5 \mathrm{~cm}$, Drim $7.7 \mathrm{~cm}$, Dbase $3.1 \mathrm{~cm}$.

$178(104.3) \quad 50 \%,>11$ fragments, complete profile, semi-fine pale pinkish brown, wheelmade with rilling internally and externally, some pink firing clouds, $\mathrm{Ht} 3.3 \mathrm{~cm}$, Drim $7.0 \mathrm{~cm}$.

$179(105) \quad$ c. 30 fragments, complete profile, semi-fine reddish-brown to buff, wheelmade, $H t 3.0 \mathrm{~cm}$, Drim $8.0 \mathrm{~cm}$, Dbase $2.9 \mathrm{~cm}$.

180 (106) 75\%, 6 fragments, semi-fine pale-greenish buff, Ht $2.6 \mathrm{~cm}$, Drim $7.0 \mathrm{~cm}$, Dbase $3.3 \mathrm{~cm}$. 
181 (107.1) complete, 1 piece, medium-soft fine pink-orange, blushing buff to orange/ pink: firing has given the effect of a buff slip, elliptical rim, Ht $3.1 \mathrm{~cm}$, Drim $7.0-7.8 \mathrm{~cm}$, Dbase $3.4 \mathrm{~cm}$.

182 (107.2) 33\%, c. 14 fragments, almost complete profile, semi-fine soft light brown with sparse mudstone inclusions, wheelmade? Dbase $3.2 \mathrm{~cm}$.

183 (108) almost complete, 14 fragments, medium-hard fine pale greenish buff, Ht $2.8 \mathrm{~cm}$, Drim $7.7 \mathrm{~cm}$, Dbase $3.0 \mathrm{~cm}$.

$184(110.2) \quad 66 \%, 4$ fragments, soft fine buff, Ht $2.7 \mathrm{~cm}$, Drim $7.4 \mathrm{~cm}$, Dbase $2.9 \mathrm{~cm}$.

185 (110.3) 75\%, 7 fragments, fine buff, Ht $3.5 \mathrm{~cm}$, Drim $7.2 \mathrm{~cm}$, Dbase $3.0 \mathrm{~cm}$.

186 (110.4) complete, 13 fragments, fine buff, uneven rim partly pulled-out, Ht $3.0 \mathrm{~cm}$, Drim $7.5 \mathrm{~cm}$, Dbase $2.8 \mathrm{~cm}$.

187 (110.5) complete, 7 fragments, fine buff, Ht 3.6-2.8cm, Drim 7.2cm, Dbase $2.8 \mathrm{~cm}$.

188 (112) 75\%, 3 fragments, medium-soft fine pale buff, $\mathrm{Ht} 2.9 \mathrm{~cm}$, Drim $7.3 \mathrm{~cm}$, Dbase $3.2 \mathrm{~cm}$.

189 (113) 75\%, 14 fragments, soft fine orange-pink, $H t 2.9 \mathrm{~cm}$, Drim $7.1 \mathrm{~cm}$, Dbase $3.0 \mathrm{~cm}$.

190 (114) almost complete, 10 fragments, semi-fine pale buff, $\mathrm{Ht} 3.4 \mathrm{~cm}$, Drim $8.0 \mathrm{~cm}$, Dbase $3.2 \mathrm{~cm}$.

191 (115) 75\%, 9 fragments, fine pale buff, trace of coil seam, moderatesoft (worn edges), Ht $3.0 \mathrm{~cm}$, Drim $7.3 \mathrm{~cm}$, Dbase $3.3 \mathrm{~cm}$.

192 (117) 66\%, 3 fragments, fine pale greenish buff, a little orange blushing, Ht $2.9 \mathrm{~cm}$, Drim $7.3 \mathrm{~cm}$, Dbase $3.4 \mathrm{~cm}$.

193 (118.1) almost complete, 4 fragments, fine orange buff, elliptical rim, $\mathrm{Ht}$ $3.5 \mathrm{~cm}$, Drim $6.5-7.7 \mathrm{~cm}$, Dbase $2.8 \mathrm{~cm}$.

194 (118.2) 50\%, 8 joining fragments and 9 small non-joining, medium-soft fine red, but firing has given impression of a buff slip, slightly thicker walls than usual, $\mathrm{Ht} 2.9 \mathrm{~cm}$, Drim $7.7 \mathrm{~cm}$, Dbase $3.4 \mathrm{~cm}$.

195 (135) 75\%, 5 joining fragments and 5 small non-joining, soft fine orange-pink, Ht $2.2 \mathrm{~cm}$, Drim $7.7 \mathrm{~cm}$, Dbase $3.0 \mathrm{~cm}$.

196 (136.1) complete, 1 piece, fine pale buff, slight orange blush, $\mathrm{Ht} 2.8 \mathrm{~cm}$, Drim $6.6 \mathrm{~cm}$, Dbase $2.8 \mathrm{~cm}$.

197 (136.2) complete, 4 fragments, soft semi-fine orange, Ht $3.0 \mathrm{~cm}$, Drim $7.1 \mathrm{~cm}$, Dbase $3.2 \mathrm{~cm}$.

198 (137) very fragmentary, not complete profile, c. 20 fragments, soft semi-fine reddish-brown, not typical in terms of fabric/firing (similar to 208) Dbase $3.0-4.0 \mathrm{~cm}$.

199 (138) complete, 1 piece, fine pale buff (orange at core), irregular rim, Ht $3.0 \mathrm{~cm}$, Drim $7.5 \mathrm{~cm}$, Dbase $3.4 \mathrm{~cm}$.

200 (139) 75\%, 11 fragments, medium-hard fine to semi-fine orange to buff, traces of coil seams, thick-walled for this type, $\mathrm{Ht} 3.1 \mathrm{~cm}$, Drim $7.4 \mathrm{~cm}$, Dbase $3.4 \mathrm{~cm}$.

201 (140) 15\%, no profile, c. 10 fragments, soft semi-fine reddish to light brown, reddish at core, Drim $8.0 \mathrm{~cm}$.

202 (141) complete, 1 piece, medium-soft semi-fine/fine orange, hardly any rilling, Ht $3.0 \mathrm{~cm}$, Drim $7.9 \mathrm{~cm}$, Dbase $3.1 \mathrm{~cm}$. 
203 (142) complete in 1 piece, fine pale green buff, $\mathrm{Ht} 3.1 \mathrm{~cm}$, Drim $7.6 \mathrm{~cm}$, Dbase $3.0 \mathrm{~cm}$.

204 (143) complete in 6 fragments, fine pale buff, Ht $2.8 \mathrm{~cm}$, Drim $8.1 \mathrm{~cm}$, Dbase $3.6 \mathrm{~cm}$.

205 (144.1) complete in 6 fragments, medium-hard fine pale greenish buff, some orange blushing, plain, quite neatly finished, some possible coil traces, small applied lump (or accidental?) at rim, $\mathrm{Ht} 3.0 \mathrm{~cm}$, Drim $7.4 \mathrm{~cm}$, Dbase $3.1 \mathrm{~cm}$.

206 (144.2) almost complete, 7 fragments, medium-soft semi-fine pale pink/brown with sparse mudstone inclusions, wheelmade, $\mathrm{Ht}$ $2.5 \mathrm{~cm}$, Drim $7.7 \mathrm{~cm}$, Dbase $3.3 \mathrm{~cm}$.

207 (144.3) 50\%, 8 fragments, soft semi-fine reddish-brown, $\mathrm{Ht} 3.0 \mathrm{~cm}$, Drim $7.5-8.0 \mathrm{~cm}$, Dbase $3.0 \mathrm{~cm}$.

$208(145) \quad$ c. 20 fragments, no profile, medium-coarse soft reddish-brown with mudstone inclusions, not typical of this deposit in terms of fabric/firing and forming.

209 (146) 33\%, 13 fragments, no profile, semi-fine pale brown, faint coil seams, Dbase $3.1 \mathrm{~cm}$.

210 (147) complete in 3 fragments, medium-hard fine pale greenish buff, faint trace of coil seam, Ht $2.8 \mathrm{~cm}$, Drim $7.2 \mathrm{~cm}$, Dbase $3.1 \mathrm{~cm}$.

211 (150) very fragmentary, almost complete profile, soft semi-fine brown fabric, atypical for this deposit - thicker walls than usual, $\mathrm{Ht}$ $3.0 \mathrm{~cm}$.

212 (159) 50\%, 8 fragments, complete profile, semi-fine pale fabric, wheelmade? Ht $3.1 \mathrm{~cm}$, Drim $8.0 \mathrm{~cm}$, Dbase $3.0 \mathrm{~cm}$.

213 (162) convex profile and broader base, semi-fine buff with sparse mudstone inclusions, wheelmade, distorted shape, $\mathrm{Ht} 3.0 \mathrm{~cm}$, Drim 7.5-8.0 cm, Dbase $3.5 \mathrm{~cm}$.

214 (163.1) almost complete, 7 fragments, medium-hard fine pale buff, well finished, Ht $3.0 \mathrm{~cm}$, Drim $7.5 \mathrm{~cm}$, Dbase $3.3 \mathrm{~cm}$.

215 (163.2) 66\%, medium-soft fine pinkish buff, thickish walls, Ht $3.1 \mathrm{~cm}$, Drim $7.0 \mathrm{~cm}$, Dbase $3.5 \mathrm{~cm}$.

216 (164) almost complete, 9 fragments, soft semi-fine orange fabric with mudstone inclusions, Ht $3.8 \mathrm{~cm}$, Drim $7.5 \mathrm{~cm}$, Dbase $2.9 \mathrm{~cm}$.

217 (165) $\quad 75 \%$ many fragments, fine reddish orange fabric, $\mathrm{Ht} 2.4 \mathrm{~cm}$, Drim $7.3 \mathrm{~cm}$, Dbase $3.0 \mathrm{~cm}$.

218 (166) 33\%, 6 fragments, medium-soft semi-fine buff, Dbase $2.9 \mathrm{~cm}$.

219 (174) almost complete, 16 fragments, soft fine pink-orange buff, $\mathrm{Ht}$ $2.5 \mathrm{~cm}$, Drim $7.6 \mathrm{~cm}$, Dbase $3.0 \mathrm{~cm}$.

220 (175) 25\%, 10 fragments, no profile, semi-fine buff-brown with darker core, possible coil seam?, Dbase $3.5 \mathrm{~cm}$.

221 (191) 75\%, c. 25 fragments, medium hard fine pale brown, $\mathrm{Ht} 3.1 \mathrm{~cm}$, Drim $7.5 \mathrm{~cm}$, Dbase $3.0 \mathrm{~cm}$.

222 (192) almost complete, 20 fragments, medium hard fine pale buff, uneven base, $\mathrm{Ht} 2.5 \mathrm{~cm}$, Drim $7.7 \mathrm{~cm}$, Dbase $3.0 \mathrm{~cm}$.

223 (193) very fragmentary, c. 20 fragments, medium hard semi-fine pale buff, Dbase $2.8 \mathrm{~cm}$.

224 (200) very fragmentary, 10 joining and 10 loose fragments, medium hard fine pale buff and orange core, Drim $7.5 \mathrm{~cm}$. 
225 (204) 50\%, 13 fragments, medium hard semi-fine pale light brown, wheel-fashioned?, Ht $2.9 \mathrm{~cm}$.

226 (208) 25\%, 15 fragments, medium hard fine pale light brown with darker core.

227 (210) $\quad 50 \%$ (5 joining base fragments and 14 non-joining), soft fine pale buff, Dbase $3.0 \mathrm{~cm}$.

228 (216.1) very fragmentary, soft semi-fine light brown, wheel-fashioned?, Dbase $3.0 \mathrm{~cm}$.

229 (216.2) very fragmentary, soft semi-fine light brown, wheel-fashioned?, Dbase $3.0 \mathrm{~cm}$.

230 (220) almost 50\%, 10 fragments, medium-hard semi-fine brown buff, Ht $2.8 \mathrm{~cm}$, Drim $7.0 \mathrm{~cm}$, Dbase $3.5 \mathrm{~cm}$.

\section{Feature 27}

Conical cups

All of the following are plain, thin-walled $(0.3-0.4 \mathrm{~cm})$, and wheel-fashioned (often with concentric striations at base), unless otherwise stated.

231 (184.1) 30\% pres, 8 fragments, complete profile, semi-fine pale buff with sparse mudstone inclusions, wheelmade with rilling, $\mathrm{Ht} 2.8 \mathrm{~cm}$, Drim $8.0-8.5 \mathrm{~cm}$, Dbase $3.2 \mathrm{~cm}$.

232 (184.2) 30\%, 9 fragments, complete profile, semi-fine pale buff with sparse mudstone inclusions, wheelmade? with rilling, very small base, $\mathrm{Ht} 3.3 \mathrm{~cm}$, Dbase $2.4 \mathrm{~cm}$.

233 (184.3) 30\%, 10 fragments, almost complete profile, semi-fine pale buff with sparse mudstone inclusions, wheelmade with rilling, exterior surface buff and interior pale, $\mathrm{Ht} 3.1 \mathrm{~cm}$, Dbase $3.1 \mathrm{~cm}$.

234 (184.4) 25\%, 2 fragments, not complete profile, semi-fine pale to reddish-brown (pale exterior and reddish-brown core), wheelmade with rilling, , Dbase $3.0 \mathrm{~cm}$.

235 (185) c. 20 fragments, not complete profile, medium-soft semi-fine pale buff, Drim $8.0 \mathrm{~cm}$.

236 (186.1) 50\%, 10 fragments, complete profile, semi-fine pale-light brown with sparse mudstone inclusions, possible coil seam visible, distorted shape, Ht 2.5-3.0cm, Drim 7.5-8.0 cm, Dbase $2.6 \mathrm{~cm}$.

237 (186.2) 50\%, 13 fragments, complete profile, semi-fine pale-light brown, wheelmade? Ht 3.0-3.3cm, Drim 7.5-8.0cm, Dbase $3.0 \mathrm{~cm}$.

238 (186.3) 25\%, 7 fragments, almost complete profile, semi-fine pale-light brown, coil join in section?, slightly thicker walls than usual and larger overall size, $\mathrm{Ht} 3.5 \mathrm{~cm}$, Drim $9.0 \mathrm{~cm}$.

239 (187) 50\%, 6 joining fragments and c. 10 non-joining, very soft semifine brown buff, Ht $3.0 \mathrm{~cm}$, Drim $7.0 \mathrm{~cm}$, Dbase $3.4 \mathrm{~cm}$.

\section{Feature 21}

240 (178) closed vessels, with flat base, 13 fragments, base only, coarse orange-buff mudstone-tempered with light grey core, wheel- 
$241(179)$

242 (182)

243 (183) kalathos, quite flaring squared rim and double horn lug on rim, 14 fragments, only rim preserved, poor preservation, soft fine pinkish brown, wheelmade with rilling internally and externally, dark brown slip internally and externally.

\section{Feature 25}

244 (203) stemmed cup, everted rim and slight carination at maximum diameter, vertical strap handle, low foot, flat underneath, fragmentary - c. 70 sherds, soft fine pink-light brown (more reddish lower and paler upper), wheelmade, dark-on-light (red to dark reddish-brown paint): probably reserved bands upper and lower and running spirals at mid-body, and band at interior rim, Ht $11.0 \mathrm{~cm}$, Dbase $3.5 \mathrm{~cm}$. (see Kythera, Tomb E, finds 3 and 4).

245 (205) hemispherical cup, 25\%, 15 fragments, not complete profile, fine pale brown/buff, wheelmade?, dark-on-light running spirals in dark brown to reddish-brown paint, reserved band on rim and lower, broad band on interior rim (dark brown); the exterior surface seems slightly burnished/well smoothed, Drim $12.0 \mathrm{~cm}$.

246 (189) tumbler, with concave walls, everted ledge rim and disk base, mainly upper part pres: c. $60 \%$ rim, c. 23 fragments, semi-fine light brown-orange with sparse mudstone inclusions, wheelmade/fashioned?, monochrome black slip all-over, two parallel grooves below rim, $\mathrm{Ht} 10.0 \mathrm{~cm}$, Drim $10.5 \mathrm{~cm}$.

247 (215) tumbler, with concave profile, flattened rim, and base/lower body slightly rounded, $60 \%,>20$ fragments, semi-coarse brown (buff) with angular mudstones, wheel-fashioned, soft-medium, monochrome orange-brown slip, 2 parallel grooves just below rim, Ht $8.5 \mathrm{~cm}$, Drim $8.0 \mathrm{~cm}$, Dbase $6.0 \mathrm{~cm}$.

248 (201) kalathos, flanged base, flaring profile and squared rim, more than half rim preserved but no lugs present, overall $66 \%$, restored from fragments, medium-hard fine pale orange buff with very sparse mudstones, wheel-fashioned, monochrome brown slip 
249 (198.2)

$250(198.1)$

251 (188)

252 (213)

$253(190)$

Conical cups

254 (194)

255 (195.1)

$256(195.2)$

257 (196) externally and internally, well-smoothed but somewhat washy slip application, $\mathrm{Ht} 8.5 \mathrm{~cm}$, Drim $16.8 \mathrm{~cm}$, Dbase $7.5 \mathrm{~cm}$.

kalathos, quite squat body and flanged base (see 201 above), rim is squared and grooved, trace of a horn lug, very fragmentary: $10 \%, 4$ fragments and almost complete profile, medium-soft fine to semi-fine orange, wheel-fashioned with rilling, smoothed monochrome brown slip externally and internally, Drim $14 \mathrm{~cm}$, Dbase $6.0-7.0 \mathrm{~cm}$.

conical bowl, small lug at mid body, $66 \%$, c. 20 joining, many non-joining fragments, soft semi-coarse pink to buff mudstonetempered, wheel-fashioned with rilling, plain, effect of a buff slip (from firing), base is badly worn on one side, Ht $10.5 \mathrm{~cm}$, Drim $28 \mathrm{~cm}$, Dbase $10.5 \mathrm{~cm}$.

blossom bowl (small), 25\%, 19 fragments, not complete profile, fine buff to light brown-pink with light grey core, wheelmade/finished?: some rilling, monochrome (black) slipped and plastic decoration, probably relief imitation of vertical leaves, walls are quite thick $(0.6-0.7 \mathrm{~cm})$. Shape similar to the larger blossom bowls, but fine fabric and probably smaller number of relief leaves around the body. Ht pres. c. 7- 8cm.

closed vessel, ovoid-conical body, probably open-mouthed, lower springing for vertical roll handle - odd attachment, lower body complete, some non-joining sherds, semi-coarse greenish buff and mudstone tempered, wheel-fashioned with rilling, dark-onlight bands and spirals, Dbase $8.5 \mathrm{~cm}$, pres. $H t 16.5 \mathrm{~cm}$. (probably ewer or jug)

closed vessel, medium-large with flat base, straight-sided lower body (cylindrical profile?), base and lower body fragmentary: c. 50 fragments, medium-coarse pink-light brown and mudstonetempered (exterior half of core: pink; interior half of core: light grey brown), coil-built, dark-on-light (black paint) two bands at lower body, some paint traces on interior rim where handle joins; Dbase $12-13 \mathrm{~cm}$. (probably low-spouted jug)

almost complete, 18 fragments, medium-hard fine pale buff with pink core and sparse mudstone inclusions, wheel-fashioned with hints of coil seams, plain, rather odd notches at rim, and applied blob of clay just inside rim, and bulge in wall $2 / 3$ of the way up, Ht $3.8 \mathrm{~cm}$, Drim $7.9 \mathrm{~cm}$, Dbase $3.1 \mathrm{~cm}$.

complete, 7 fragments, fine/semi-fine orange with sparse mudstone inclusions, wheel-fashioned with traces of coil seams, plain, thickish walls, $\mathrm{Ht} 3.1 \mathrm{~cm}$, Drim $7.7 \mathrm{~cm}$, Dbase $3.7 \mathrm{~cm}$.

complete, 20 fragments, fine silty pink-orange with sparse mudstone inclusions, wheel-fashioned, plain, thickish walls, $\mathrm{Ht}$ $3.0 \mathrm{~cm}$, Drim $7.7 \mathrm{~cm}$, Dbase $3.4 \mathrm{~cm}$.

$33 \%, 10$ fragments, fine buff (pink at core) with some large mudstone inclusions, wheel-fashioned with coil seams visible, plain, Ht $3.0-3.5 \mathrm{~cm}$, Drim $7.0-8.0 \mathrm{~cm}$. 
258 (197) complete, 5 fragments, semi-fine pink with sparse mudstone and calcareous inclusions, wheel-fashioned, plain, thickish walls, $\mathrm{Ht}$ $3.5 \mathrm{~cm}$, Drim 7.7-8.0 cm, Dbase $3.9 \mathrm{~cm}$.

259 (207) c. 20 fragments, soft semi-fine to medium orange-brown with sparse mudstone inclusions, plain. 


\begin{tabular}{|c|c|c|c|c|c|c|c|c|c|c|}
\hline SOYSE IIEUS & & & & & & & & - & & \\
\hline вұКЧч & & & & & & & 一 & & & \\
\hline 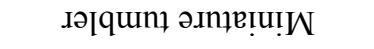 & & & & & & & & & - & \\
\hline 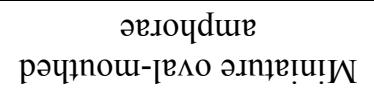 & & & & & & & & & - & \\
\hline 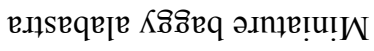 & & & & & & & & $\sim$ & 一 & \\
\hline SłоdıəмоІІ & & & & & & & & & 一 & \\
\hline 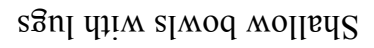 & & & & & & & $\sim$ & & & \\
\hline IMoq/dn $\mathcal{D}$ & & & & & & & & -1 & & \\
\hline sdno pə⿱nods MOI[एบS & & & & & & & & $\sim$ & & \\
\hline sdno n!џرəX & & & & & & & - & & & \\
\hline sdnว IIə⿱ & & & & & & & $m$ & $m$ & & \\
\hline SəłPId & & & & & & & 一 & 一 & $\Xi$ & \\
\hline Sıəรนวつ & & & & & & & 一 & $m$ & 一 & 一 \\
\hline S.əIZE.ı & & & & & & & $N$ & $m$ & 一 & 一 \\
\hline sdure 1 & & & & & & & 一 & N & $\mathrm{N}$ & 一 \\
\hline 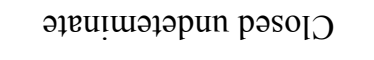 & $N$ & N & & & $N$ & $m$ & $\nabla$ & in & - & 一 \\
\hline S.tẹ .te[nqoID & iे. & & & & & & & & & \\
\hline S.əМ日 & $N$ & & 一 & & & & 二 & - & & $m$ \\
\hline Sôn! pəłnods-MoT & $\mathrm{N}$ & & & & & & $a$ & $m$ & & 一 \\
\hline s[Moq wossolg & - & & 一 & & - & & $n$ & 一 & & 一 \\
\hline 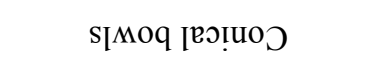 & - & & 一 & & - & & $m$ & & n & $\infty$ \\
\hline su!seq рәгрuвч-оิu!y & $\mathrm{N}$ & & 一 & & & & 6 & $m$ & & 一 \\
\hline Iочџе[ЕХ & - & & $\mathrm{N}$ & & $\sim$ & 一 & $m$ & & & 一 \\
\hline S.Іәqun ${ }_{\mathrm{L}}$ & - & & - & & $\sim$ & & 6 & - & - & \\
\hline sdno [вə!̣əપds!̣யәН & $\sim$ & & & & - & & $n$ & in & 6 & \\
\hline sdn॰ pәшшәฺS & & & 一 & & - & & $N$ & $\stackrel{2}{-}$ & & \\
\hline \multirow[t]{2}{*}{ sdno [שว!̣oग } & ป & $\sim$ & $\frac{1}{1}$ & $a$ & 6 & & $\hat{a}$ & す & $\hat{N}$ & t \\
\hline & 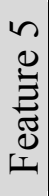 & 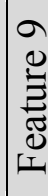 & 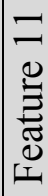 & 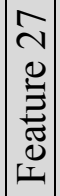 & 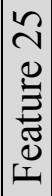 & 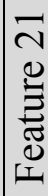 & 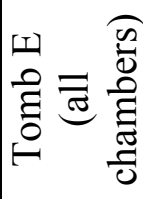 & है & 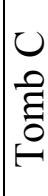 & 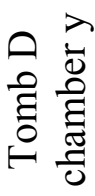 \\
\hline
\end{tabular}




\section{Pottery Analysis}

\section{Conical cups}

These are by far the most common shape in the ceramic assemblage. Out of 259 catalogued pots, 218 are conical cups, comprising the vast majority of the pots recovered from the whole excavated area. There are two clearly distinguishable types. The first type, Type A (FIGS. 12, 15-16, PLATES 1a-c, 2c), tends to be 3.5$4.0 \mathrm{~cm}$ in height, with a rim diameter of c. $8 \mathrm{~cm}$ and a base diameter of c. 3.5$4.0 \mathrm{~cm}$. It is standardised not only in size but also in shape, with a slightly convex profile and often a differentiated base. In terms of technology the type consistently has relatively thick walls, and in some cases traces of coil seams can be identified in the break and on the interior surfaces (PLATE 1a). Such traces point quite clearly to the use of a wheel technique that involves coils, which can be most conveniently dubbed 'wheel-fashioning', as opposed to 'wheelthrowing'. ${ }^{14}$ The fabric is a local fine to semi-fine buff to light brown and more rarely reddish-brown, sometimes with sparse mudstone inclusions (see below, Comment on fabrics), and the vessel surfaces are always plain and undecorated.

The second type, Type B (FIGS. 12, 18-19, PLATES 1a-c) is noticeably smaller than the first type in all its dimensions, with an average height of $\mathrm{c.} 3 \mathrm{~cm}$, a variable rim diameter but averaging c. $7.5 \mathrm{~cm}$, and a base diameter of c. $3 \mathrm{~cm}$. Its shape also differs in being strictly conical, and in terms of technology it is easily distinguished from Type A, especially in its considerably thinner walls. Traces of coil seams are much harder to identify, which makes it difficult to be sure if the technique used is indeed wheel-fashioning. If it is, then in producing this type, potters were taking more time to regularise surfaces and thin vessel walls, thereby 'covering their tracks', be it inadvertently or not. The fabric is similar to that of Type A, but tends to be somewhat paler, probably reflecting different firing conditions (higher temperature?).

There is a clear pattern in the distribution of these two types. Types A and B were never found together in the same feature. All conical cups from feature 5 belong to Type A while the ones from features 11, 27 and 9 belong to Type B. The conical cups found in feature 25, although looking rather similar to those of Type A in terms of shape (FIG. 20), tend to be smaller than typical Type A size. The clear differentiation in size between the conical cups of different features is illustrated in the scatterplot of figure 12 .

The distinction between these types of conical cups could reflect a chronological shift (and hence a different relative date for the two main features 5 and 11) or could be attributed to the existence of different production traditions (potters/workshops) or a combination of both. In fact, while very few conical cups were fully published by Coldstream and Huxley, there is reasonable comparative evidence - from their LM IA deposits $\eta$ and $\theta$, and LM IB deposits $\mu$,

\footnotetext{
${ }^{14}$ These techniques are explained in V. Roux and M.-A. Courty, 'Identification of Wheel-fashioning Methods: Technological Analysis of $4^{\text {th }}-3^{\text {rd }}$ Millennium BC Oriental Ceramics', Journal of Archaeological Science 25 (1998), 747-63. In a Cretan context, C. Knappett, 'Technological Innovation and Social Diversity at Middle Minoan Knossos', in W. Cavanagh and E. Hatzaki (eds.), Knossos: Palace, City, State (BSA Studies; London, in press).
} 
$v$ and $\xi$ in the Kastri settlement - to support the chronological explanation. First, the conical cups from the 1960s excavations display differences in their dimensions: those from LM IA are larger than those from LM IB, and the sizes tally very well with the two types we have described above. ${ }^{15}$ Second, the key question of wall thickness is also raised by Coldstream and Huxley, when they remark upon LM IB conical cups that "the fabric is generally finer and thinner than in LM IA". 16 This too corresponds very well with our own observations on the two different conical cup types. Third, they note a difference in shape, with the LM IB examples being strictly conical but those of LM IA tending to turn slightly inwards at the rim. ${ }^{17}$

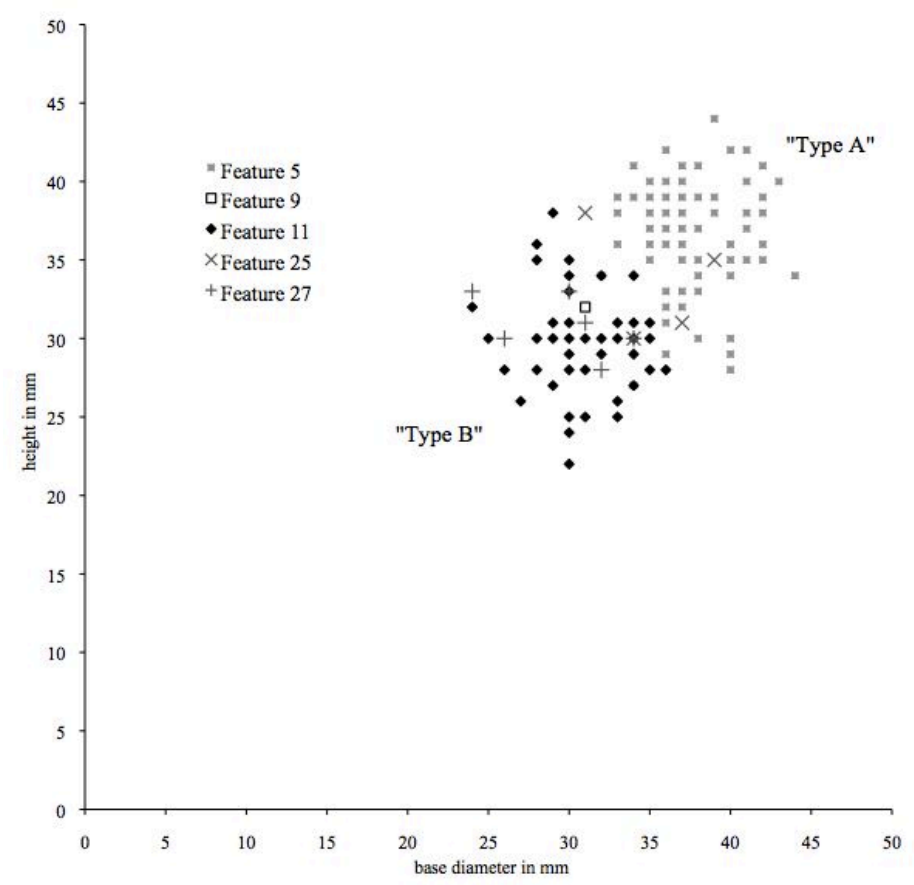

Fig.12 Scatterplot showing the dimensions (height and base diameter) of conical cups from different Tholos deposits.

\section{Stemmed cups}

There are two definite examples from Tholos: one each from features 11 and 25 . That from feature 11 (144, FIG. 17) has a definite stem, while the one from feature 25 (244, FIG. 20) has a foot rather than a true stem. Both stemmed cups from Tholos are in a fine, rather soft, light-coloured fabric, not dissimilar to the local fabric of conical cups, although finer. The two examples have dark-on-light painted decoration, although each of them bearing different decorative motifs $\mathbf{1 4 4}$ has lilies (FIG.17) while $\mathbf{2 4 4}$ has running spirals. The decoration of $\mathbf{2 4 4}$ appears typical of LM IA, and this early dating is further supported by the vessel's indistinct stem. In contrast, 144 seems much more likely to belong in LM IB.

\footnotetext{
${ }^{15}$ For Deposit $\eta$ (LM IA), see Kythera, 116, no. 31; for Deposit $\theta$ (LM IA), Kythera, 122, no. 16; for Deposit $\mu$ (LM IB), Kythera, 130-1, no. 28; for Deposit v (LM IB), Kythera, 136, no. 29 Deposit $\xi$ (LM IB) - p.144, no. 90.

${ }^{16}$ Kythera, 131.

${ }^{17}$ Kythera, 285 (LM IA summary) and 294 (LM IB summary).
} 
Stemmed cups so far occur on Kythera exclusively in tombs, and even then only rarely and, like ours, seem to be local products. ${ }^{18}$ Coldstream and Huxley declare that the stemmed cup is new to Kythera in LM IB, although this is not based on stratigraphical evidence. ${ }^{19}$ On Crete, stemmed cups certainly occur in LM IB, but are also found in LM IA contexts. ${ }^{20}$

\section{Hemispherical cups}

Remarkably, given the general ubiquity of this type in settlements, only one definite and two possible examples, all fragmentary, are present at Tholos. 245 from feature 25 (FIG. 20) is in a local fine pale buff fabric and has dark-on-light retorted spirals in a matt paint. It is clearly of LM IA type. A parallel of sorts come from Deposit $\theta$, no. 7 (dark-on-light spirals, but not retorted). ${ }^{21}$ Coldstream and Huxley call this type the 'rounded cup', saying that it becomes popular in LM IA. They change markedly in LM IB in shape and, of course, in decoration. ${ }^{22}$ Fragments of two more cups with everted rim and thin vertical strap handle were recovered from feature 5 (1-2, FIG. 13). Due to their fragmentary state, it is not possible to identify them with certainty as hemispherical cups, and they could even be stemmed cups. No decorative motifs were preserved on their worn surface to provide clues as to their date.

\section{Tumblers}

This shape is represented by one example each from features 5 (3, FIG. 13) and 11 (145, FIG. 17), and two from feature 25 (246-247, FIG. 20). Coldstream and Huxley found eight more in Tombs C, E and J (see FIG. 11). All examples are similar in having an all-over dark slip, which is an otherwise uncommon surface treatment in LM I (see comments below on both blossom bowls and kalathoi), and incised grooves below a slightly everted, flattened rim. However, their profile varies between conical and concave, and the base can either be flanged or bevelled. Some variation appears in their fabric, both in terms of colour (light brown to orange) and coarseness (fine to medium coarse mudstone-tempered). One might assume that some of the variation is chronological in nature, but even within a single context at Tholos (feature 25) the two tumblers that are present are quite different to one another (FIG. 20). They are all wheel-made but there is no clear evidence to distinguish between wheel-thrown or wheel-fashioned techniques. While Coldstream and Huxley indicate that the type belongs in the LM IA period, in the current contexts (if the suggested dating of the features is

\footnotetext{
${ }^{18}$ e.g. Kythera, 243-4, 253, fig. 87, pl. 75.

${ }^{19}$ Kythera, 294.

${ }^{20}$ Some of the Cretan examples have pierced bases and thus were used as rhyta; those from Kythera do not possess this feature. Evans shows Cretan stemmed cups of both LM IA and LM IB date - A.J. Evans, The Palace of Minos at Knossos, IV, (London, 1935), 363-4, fig. 304. The one he illustrates as belonging to LM IA is actually from Palaikastro - see also R.C. Bosanquet and R.M. Dawkins, The Unpublished Objects from the Palaikastro Excavations 1902-1906, Part I, (BSA supp. Paper 1; London, 1923), pl. 17b. Indeed, the Palaikastro LM IA example (with typical LM IA decoration of medallion spirals) has a foot more like our 244 (the decoration of which we suppose to be LM IA), whereas Evans' LM IB example (fig. 304c, in fact from Phaistos) resembles our 144 (which we suppose to be LM IB judging by its decoration) in its stem.

${ }^{21}$ Kythera, 121.

${ }^{22}$ Kythera, 293.
} 
correct) we may have examples from both LM IA and LM IB. This shape skeuomorphs stone (serpentinite) prototypes. $^{23}$

\section{Kalathoi}

Six kalathoi were recovered from the Tholos deposits; one from feature 5 (4, FIG. 13), two from 11 (146-7, FIG. 17), one from 21 (243) and two from 25 (248-9, FIG. 20, PLATE 1i). Coldstream and Huxley found none in the settlement deposits, but three in Tomb $\mathrm{E}^{24}$ and two in Tomb ; $^{25}$ they describe the kalathoi found as having "a pronounced concavity in the profile, and a narrow foot with torus moulding". ${ }^{26}$ All the Tholos examples have these features too, but there is some variability in shape: $\mathbf{1 4 6}$ is tall, slender, with an extremely concave profile, while 4 and 248 are broader and less concave. There may even be a chronological aspect here, since 146 is from feature 11 (LM IB? context), while 4 and 248 are from features 5 and 25 respectively (both LM IA?). ${ }^{27}$ While no stone or metal versions of this shape are known, certain elements of the shape, detail and surface treatment may conceivably be skeuomorphic. All variants regardless of shape details are treated with an all-over dark slip, a treatment similar to that used on ceramic blossom bowls and tumblers to imitate stone versions. Likewise, horn lugs at the rims of most examples are similar to those found on stone bowls. ${ }^{28}$ In contrast, Coldstream and Huxley point to the sharply-defined rim and torus foot as imitative of metal prototypes. ${ }^{29}$ All present examples are locally made, in fine to semi-fine orange-buff to light brown fabrics. They are all wheel-made, although it is not clear if they are wheel-fashioned or wheelthrown. Coarser versions of this form were made in Crete, Melos and Mycenae, but close parallels to the Kytheran type are lacking. ${ }^{30}$

\section{Ring-handled basins}

Ceramic versions of this type are unknown elsewhere in the Aegean, but there are three from Tholos: two come from feature 5 (nos. 5-6, FIG. 13, PLATES 1h, 2g) and one from feature 11 (148, FIG. 17). They show some variation in form. They are all in a similar semi-fine to medium-coarse mudstone-tempered fabric. All three examples seem wheel-fashioned and have plain surfaces, while two (5 and 148) have deep notches at the handle and the rim. This shape is not known from the 1960s settlement excavations but a number were found in the Kastri tombs (see FIG. 11).

\footnotetext{
${ }^{23}$ see P.M. Warren, Minoan Stones Vases (Cambridge, 1969), 98: tankards = type 40.

${ }^{24}$ Kythera, 245-246 (nos. 17-19).

${ }^{25}$ Kythera, 234 (no. 7) and 236 (no. 26).

${ }^{26}$ Kythera, 286.

${ }^{27}$ The examples reported by Coldstream and Huxley come solely from deposits assigned to LM IA, with no mention of any continuation into LM IB.

${ }^{28}$ e.g. Kythera, pl. 82, 8-9.

${ }^{29}$ Kythera, 286.

${ }^{30}$ The Cretan parallels referred to are from the Magazine of the Lily Vases in A.J. Evans, The Palace of Minos at Knossos, I (London, 1921), 577, fig. 421, 8; and from the House of the Sacrificed Oxen, A.J. Evans, The Palace of Minos at Knossos, II (London, 1928), 304, fig.176L. The Knossian parallels are very broad; they simply show that the general shape was known on Crete.
} 
Ceramic ring-handled basins are quite clearly skeuomorphic, copying metal prototypes. While there are no metal parallels known from the island, ${ }^{31}$ there are many from the rest of the Aegean. At Mycenae they are found in the Shaft Graves, but on Crete they come mainly from non-funerary LMI contexts, for example at Knossos, Mochlos and Zakros. ${ }^{32}$ However, the most remarkable parallel for the Tholos examples is not in the form of an actual vessel but a depiction of such an object on a wall-painting at Akrotiri on Thera, from Xeste 3, Room 3b ground floor. $^{33}$ It shows a youth carrying a ring-handled basin (yellow in colour, probably representing metal or ceramic?). Not only is the shape very similar, but even the details of the notches at the handle are clearly rendered (none are visible at the rim).

\section{Conical bowl}

There are at least three examples among the present deposits, all quite worn and degraded, one each from features 5 (7, FIG. 14), 11 (149) and 25 (250, FIG. 20). They are all plain in form. $\mathbf{2 5 0}$, and probably $\mathbf{7}$, each have a small lug at the midbody, akin to that on an example published by Coldstream and Huxley from Tomb D. ${ }^{34}$ They are both in a medium-coarse light reddish-brown mudstonetempered fabric; vessel $\mathbf{7}$ seems to be coil-built and $\mathbf{2 5 0}$ is wheel-fashioned. The example from feature 11 is quite fragmentary and in a finer light brown fabric.

From Tomb D (chamber g) there are actually 18 examples in all, ${ }^{35}$ emphasising the relative paucity in our deposits. These are dated to both MM IIIB and LM IA by Coldstream and Huxley, who note that the later versions tend to be deeper. ${ }^{36}$ They also continue into LM IB with very little change. ${ }^{37}$

\section{Blossom bowls}

Three examples of this shape come from the Tholos deposits: one each from features 5 (8, FIG. 13), 11 (150) and 25 (251). Coldstream and Huxley recorded 5 examples from Tomb E (see FIG. 11), which led them to attribute the use of these ceramic vessels solely to LM IA, but (if the dating of the Tholos features is accepted) our examples seem to belong to both LM IA and LM IB, a broader date range that is not particularly surprising. The Tholos examples have an all-over dark slip, and very thick body walls, both features referencing stone prototypes. They appear to be coil-built and with some rotative assistance, and the relief decoration of lily petals is executed in appliqué Their fabric is a semi-coarse, light reddish-brown and mudstone tempered; 251, however, is much smaller and in a paler and finer fabric.

\footnotetext{
${ }^{31}$ Though the shape is not dissimilar to that of the silver cups from the Kastri settlement - Kythera, pls.59.23-4, 60.23-4.

${ }^{32}$ Evans (n. 30), vol. II, 624, 637-644, figs. 402-3, 407, 409B; H. Matthäus, Die Bronzegefässe der kretisch-mykenisch Kultur (PBf II, 1, 1980). Matthäus has very useful information on ring-handled basins, which he terms Breitrandschalen, from all of the abovementioned contexts, such as Mycenae etc. His work supersedes to a large extent H. Catling, 1964 Cypriot Bronzework in the Mycenaean World, (1964), e.g. 174, form 10.

${ }^{33}$ C. Doumas, 147, 151, pls. 111, 115; The Wall-paintings of Thera (Athens, 1992).

${ }^{34}$ Kythera, 237, no. 31 (pl. 71).

${ }^{35}$ Kythera, 237-238, nos. 28-38.

${ }^{36}$ Kythera, 286.

${ }^{37}$ Kythera, 294.
} 
Just as the ceramic skeuomorphs of metal ring-handled basins are unique to Kythera, so too are the ceramic skeuomorphs of stone blossom bowls. ${ }^{38}$ Several stone examples were found in tomb $\mathrm{L}$ at Kastri and Warren notes more than 120 known examples from Crete and elsewhere, all of serpentinite. ${ }^{39}$ The ceramic versions seem to copy the most common style of decoration in which the individual petals of the blossom decoration are defined by a distinct midrib. As we have seen above, this shape is not the only pottery type in the Kastri tomb assemblages to mimic a stone prototype. ${ }^{40}$

\section{Low-spouted jugs}

Two almost complete examples of this shape come from Tholos feature 5 (9-10, PlATES 1e, 2c). The term 'low-spouted jug' was coined by Coldstream and Huxley ${ }^{41}$ to describe what we might otherwise choose to call an open-mouthed jug. ${ }^{42}$ The examples they present are practically identical to those published here. ${ }^{43}$ Both the mouth and the base are relatively wide, with a body profile that is gently convex from base to rim. The type has a trough spout that only rises slightly above the level of the rim, opposite a vertical roll handle that is attached either at the rim or as much as $2 \mathrm{~cm}$ below it. This latter feature is quite distinctive and tends on the whole not to be a feature amongst Cretan jugs of this period. The construction technique of low-spouted jugs is also of interest - both examples catalogued here (9-10) are coil-built with little if any sign of rotative assistance. With regard to fabric these vessels are invariably local, in a semicoarse buff fabric tempered with mudstone inclusions. Their dark-on-light decoration consists of bands at the lower body, mid-body and rim, and at the maximum diameter two large running spirals, one on each side of the vessel between handle and spout. According to Coldstream and Huxley this type only occurs in LM IA contexts, particularly tombs, and this observation would appear to be borne out in the present material too, in which low-spouted jugs do occur in feature 5 (putatively LM IA) but not in feature 11 (putatively LM IB).

\footnotetext{
${ }^{38}$ Coldstream and Huxley (Kythera, 286) have the following to say about LM IA blossom bowls: "of peculiar interest are our local imitations of closed stone bowls. Serpentine blossom bowls, decorated with six lily petals in relief, were imported from Crete to Kythera (L 13-16); the closest - and perhaps therefore the earliest - of the clay imitations try to reproduce the heavy thick walls and the grey soapy surface of the originals (D 9-10, E 22). The others are made in the normal technique; yet when the lily petals are gouged out of the surface (E 20, J 16), the illusion of stone is still preserved. Latest, perhaps, are E 23-4, where the potters have resorted to a time-saving device in adding the petals by the appliqué technique; furthermore, the collar and disc foot are foreign to the serpentine prototype."

${ }^{39}$ Warren (n. 23), 14-17.

${ }^{40}$ Further discussion of the ceramic skeuomorphs of stone vessels on Kythera can be found in A.H. Bevan, 'Value Regimes in the Eastern Mediterranean Bronze Age: A Study Through Stone Vessels' (unpublished PhD diss., UCL 2001), 235-6.

${ }^{41}$ Kythera, 287.

${ }^{42}$ Coldstream and Huxley contrast it to the 'high-spouted jug', a feature of both MM IIIB and LM IB but not LM IA, and a shape that might more commonly be dubbed 'beaked jug' or 'jug with cutaway spout'.

${ }^{43}$ Particularly close is no. 59 from Tomb E (Kythera, pl. 79). Vessel dimensions are also informative: in height the 12 examples published by Coldstream and Huxley vary between $16.5-21.4 \mathrm{~cm}$, Drim 12 $15.6 \mathrm{~cm}$, and Dbase $6-11 \mathrm{~cm}$. The two published here have heights of 20.1 and $22.2 \mathrm{~cm}$, Drim of 13.5 and $14.5 \mathrm{~cm}$, and Dbase of 10 and $10.8 \mathrm{~cm}$. They thus fall substantially within the very same size range. Moreover, they also have rather elliptical rims, a feature of at least two of those published in 1972.
} 


\section{Ewers}

There are three examples of this shape from Tholos: two definite ones from feature 5 (11-12, FIG. 14, PLATES 2e-f) and one possible from feature 11 (151, FIG. 17). ${ }^{44}$ The round-mouthed ewer is similar to the low-spouted jug in many ways. It is in essentially the same local fabric, a semi-coarse buff that is mudstonetempered, and receives the same sort of dark-on-light decoration of bands and large running spirals. It has a single vertical roll handle, always attached at the rim proper, but its rim is perfectly round with not even the hint of a spout. Its body profile is ovoid-conical, not unlike that of contemporary ewers, ovalmouthed amphorae and stirrup jars from Crete. As for forming technique, there is some variability - $\mathbf{1 2}$ and $\mathbf{1 5 1}$ are both coil-built, whereas $\mathbf{1 1}$ is wheelfashioned (see PLATE 2f).

Coldstream and Huxley publish a number of examples, mainly from tombs (FIG. 11). Although they are common in LM IA, and those from Tomb E almost certainly belong in this period, the type actually begins in MM IIIB. However, the earlier examples tend to have a torus foot, and of course decoration more characteristic of MM IIIB, notably tortoiseshell ripple. ${ }^{45}$ The LM IA examples have "slim ogival proportions", and a higher neck. ${ }^{46}$ Interestingly, one of the most exemplary pieces from Tomb D (no.44, pl. 72) is almost identical in shape, size ${ }^{47}$ and decoration to $\mathbf{1 1}$ from feature 5 (further support for a date in LM IA for this context). Evidence at Tholos for ewers in LM IB is less convincing: 151 from feature 11 may be a ewer, but this is far from certain given that its upper body is not preserved. Moreover, given the fact that it is hand-made, and has spiral decoration not dissimilar to that commonly seen in LM IA, it may be an LM IA survival.

\section{Perforated jug}

Vessel 141 (from feature 9) is a one-off in the deposits presented here. It has a perforated shoulder, but unfortunately no neck or rim is preserved (FIG. 20, PLATE 1g). It has plain surfaces, is wheel-fashioned, and its fabric is a local semicoarse mudstone-tempered orange buff. There would appear to be a parallel for the perforated neck from Tomb E, ${ }^{48}$ and while the latter example does not have any lower or mid-body preserved, it does have the neck and rim, as well as a handle attachment on the shoulder. This suggests that the Tholos fragment may also have had a vertical handle (and hence be a jug rather than a jar). Coldstream and Huxley suggest that a perforated vessel of this type may have served as a censer for burning aromatic substances (although there is no sooting on the interior of 141).

Seven additional closed vessels of indeterminate type were from the Tholos deposits were catalogued. Only their lower parts were preserved. They seem to

\footnotetext{
${ }^{44}$ No. 151 does not have any neck or rim, but it seems rather certain to be a ewer due to its body form (FIG.16).

${ }^{45}$ Kythera, pl. 72: 61, 63.

${ }^{46}$ Kythera, 287.

${ }^{47}$ No. 44 from Tomb D - Ht 32cm, Drim $12 \mathrm{~cm}$, Dbase $6 \mathrm{~cm}$. No. 149 from feature $5-\mathrm{Ht} 32 \mathrm{~cm}$, Drim $11.5 \mathrm{~cm}$, Dbase $9.3 \mathrm{~cm}$.

${ }^{48}$ Kythera, no. 43 , on p. $248-9$, and pl. 77 , fig. 89.
} 
be either low-spouted jugs or ewers in the local mudstone-tempered fabric. $\mathbf{1 3}$ and $\mathbf{1 7}$ from feature 5 are coil-built; $\mathbf{1 4 0}$ from feature 9, being finer in fabric, is wheel-fashioned; 240 and $\mathbf{2 4 1}$ from feature 21 are wheel-fashioned; and $\mathbf{2 5 2}$ and 253 from feature 25 are wheel-fashioned and coil-built, respectively. Finally, as reported above (see Excavated Features), possible fragments of a brazier and an alabastron or rhyton were also discovered, but it should be stressed that these identifications cannot be confirmed.

\section{Comment on fabrics}

All catalogued vessels and the majority of loose sherds were of mainly two fabric types, closely related to each other. The first is a fine to semi-fine, buff to reddish-brown fabric occasionally containing sparse dark red angular mudstone inclusions. The second fabric, being medium in coarseness, seems to be of the same clay type but regularly tempered with angular dark red to dark brown mudstone inclusions. Both fabrics are common among the Proto- and Neopalatial pottery at Kastri and have proved to be of local production. ${ }^{49}$ Nevertheless, among the uncatalogued pottery sherds there was a small number of abraded fragments of different fabrics. A few sherds both of orange and dark red micaceous fabrics, typical of Early Bronze Age and Neopalatial Kastri respectively, were found in most excavated deposits. The Early Bronze Age date of some of this material was confirmed by stylistic features, such as incised herringbone pattern, slashed horizontal handle, vertical raised handle with incised decoration (PLATE 2b), foot bases and thickened-rim jars with plastic rope decoration. ${ }^{50}$ Furthermore, there were some sherds of a medium coarse sand-tempered fabric, typical of Prepalatial and, to a lesser extent, of Protopalatial Minoanising pottery at Kastri. Among the latter it is worth mentioning a horizontal pushed-through handle, typical of Kastri deposit $\gamma .{ }^{51}$

\footnotetext{
${ }^{49}$ E. Kiriatzi, 'Sherds, fabrics and clay sources: reconstructing the ceramic landscapes of prehistoric Kythera', paper to be presented at METRON conference, University of Yale, New Haven, April 2002.

${ }^{50}$ See pottery from Kastri Deposit $\alpha$ : Kythera, 75-83.

${ }^{51}$ Kythera, 93, and pl. 21, nos. 25-29.
} 

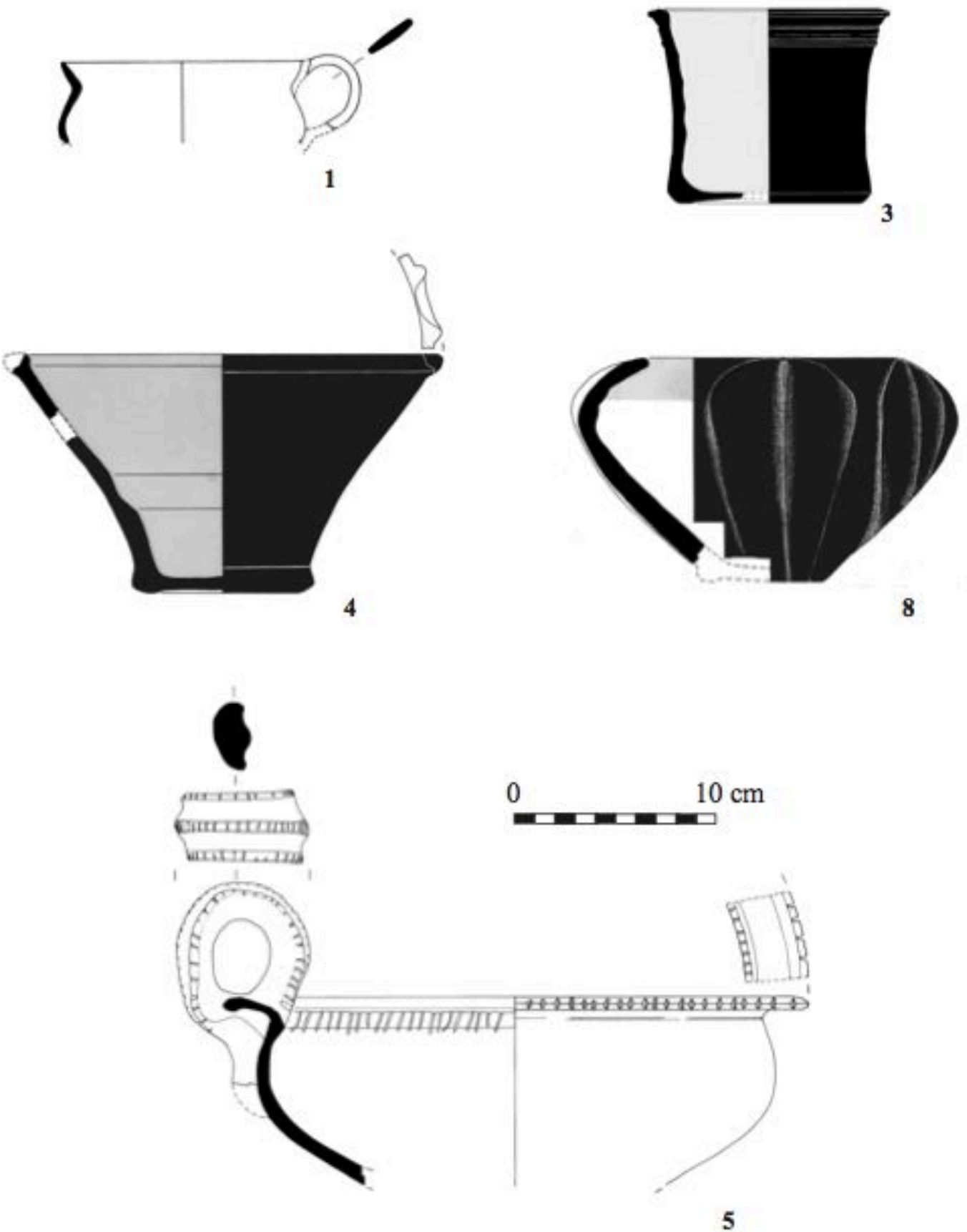

Fig.13 Catalogued pottery from feature 5. 

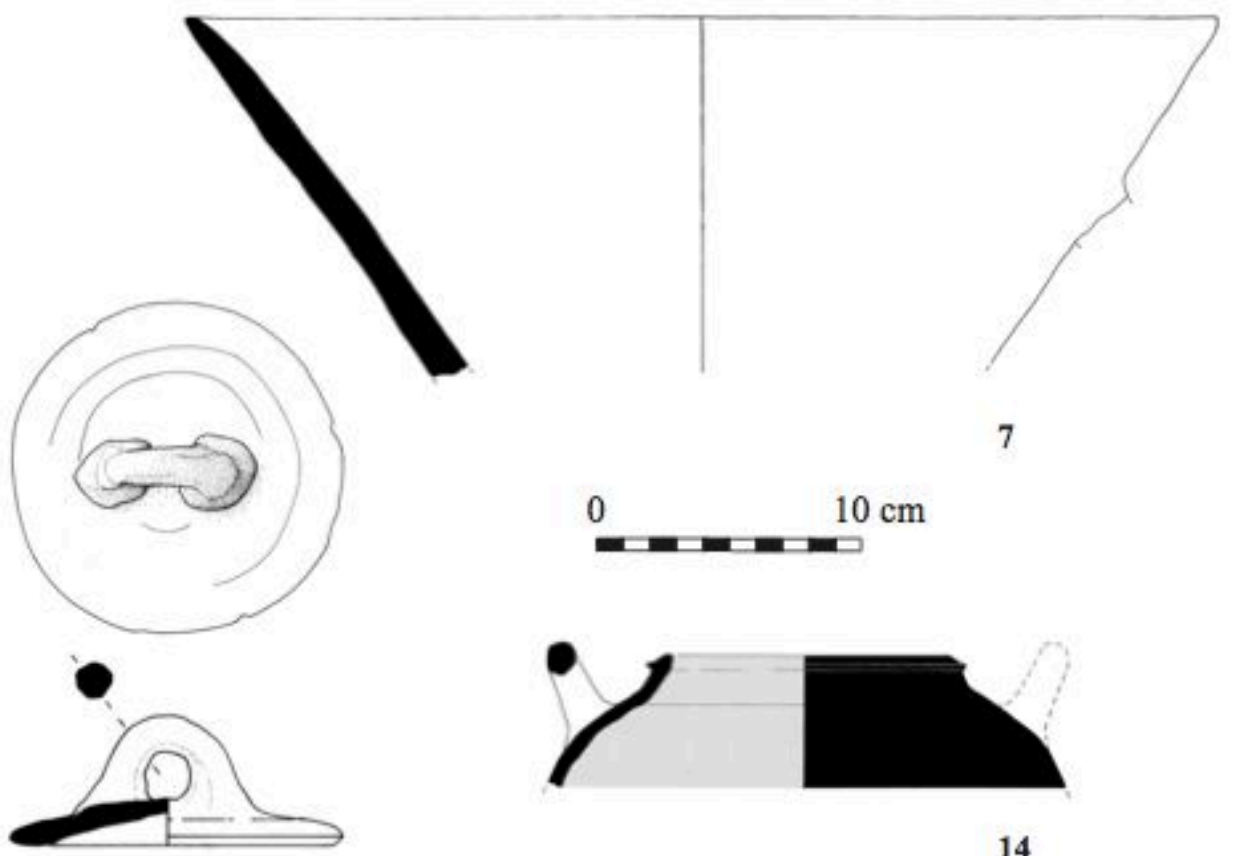

16
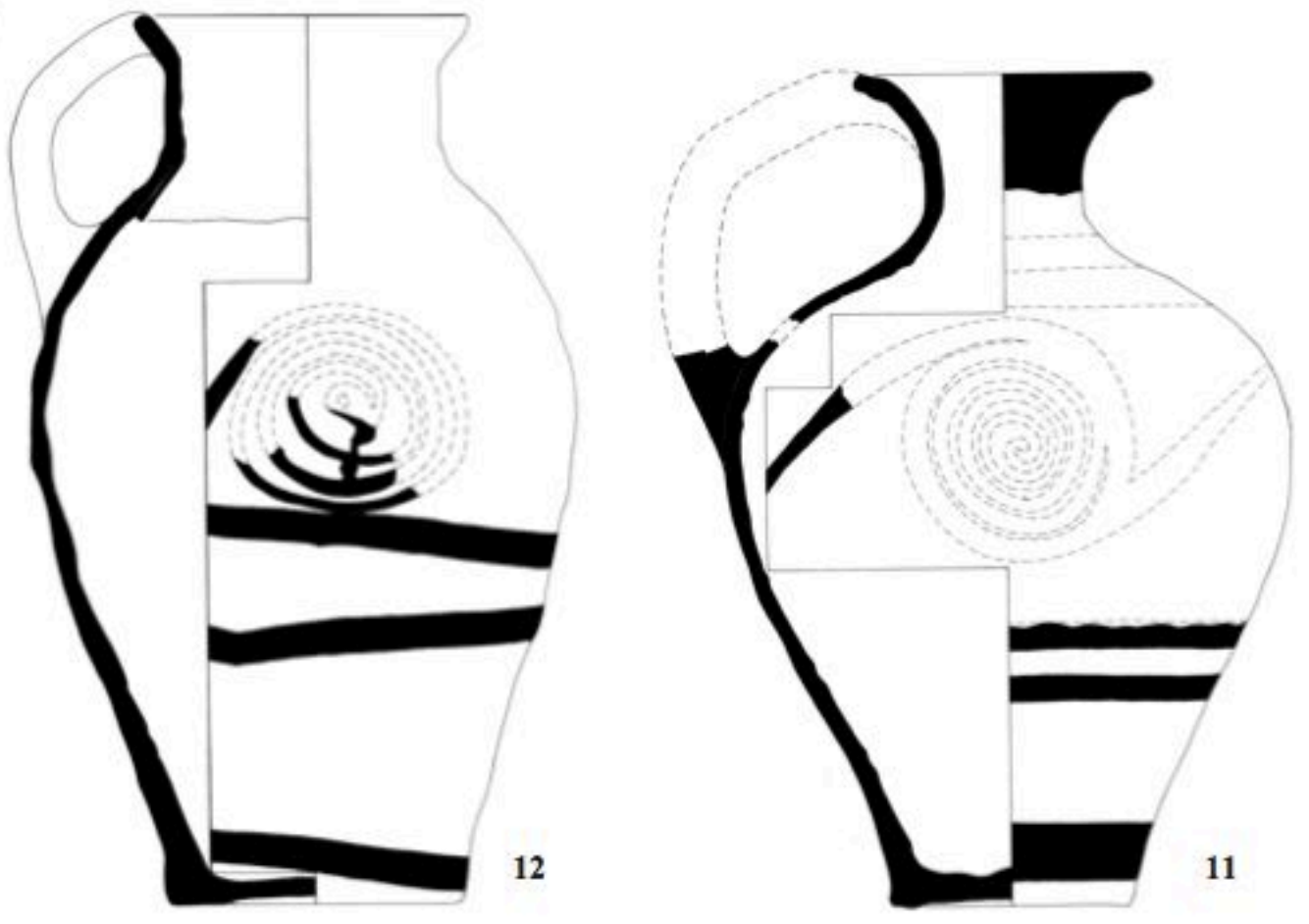

Fig.14 Catalogued pottery from feature 5. 


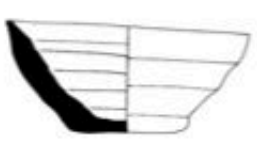

15

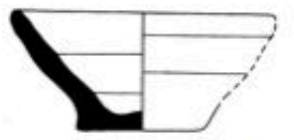

36

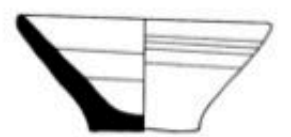

51

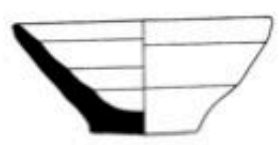

59

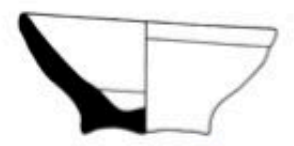

75

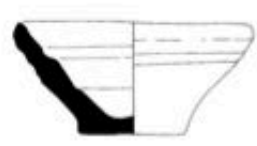

19

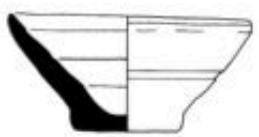

38

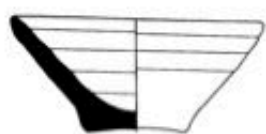

54

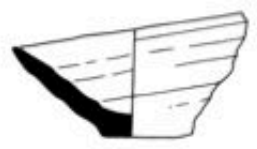

60

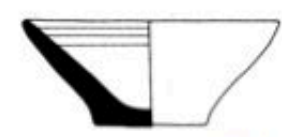

84

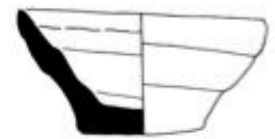

32

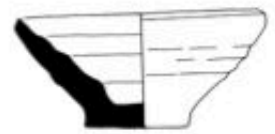

41

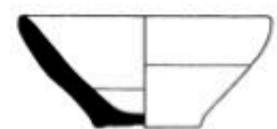

55

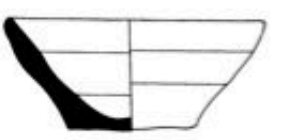

67

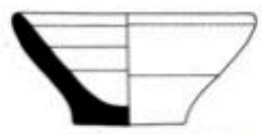

88

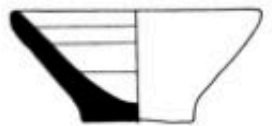

57

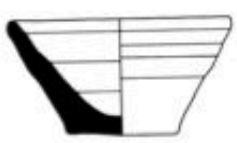

72

$10 \mathrm{~cm}$

Fig.15 Catalogued pottery from feature 5. 


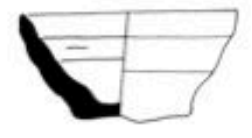

90

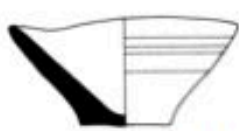

107

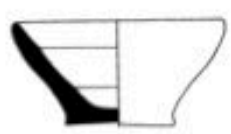

118

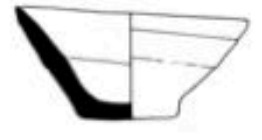

98

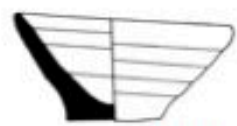

110

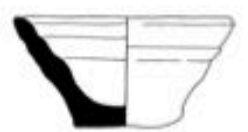

120
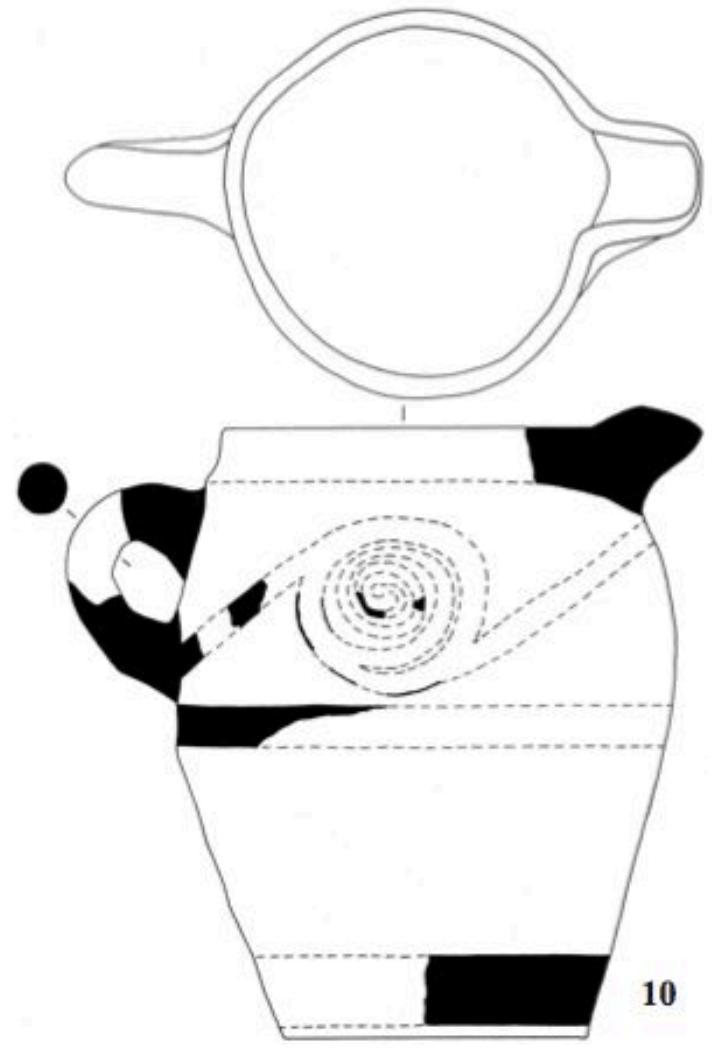

Fig.16 Catalogued pottery from feature 5.
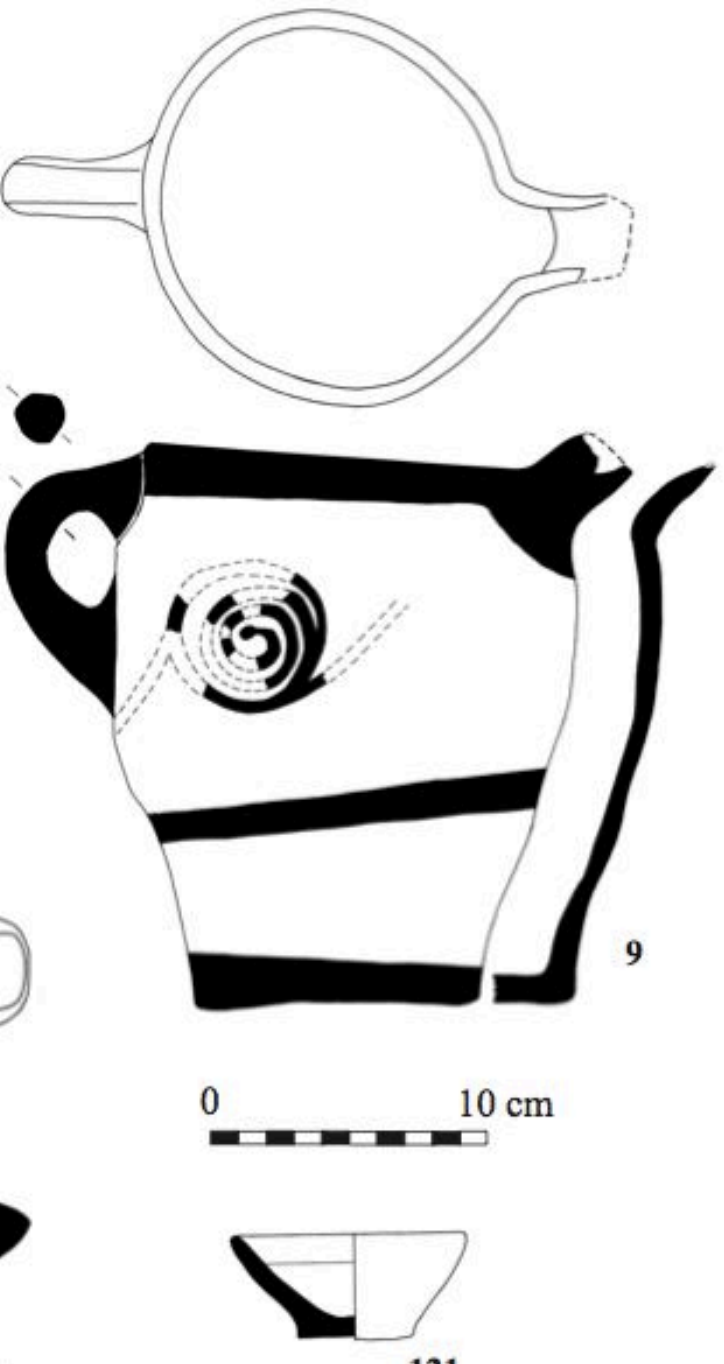

121

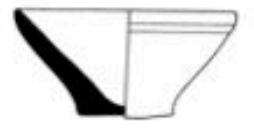

123

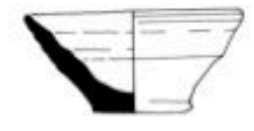

130

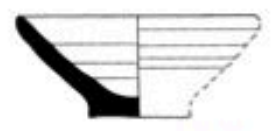

133

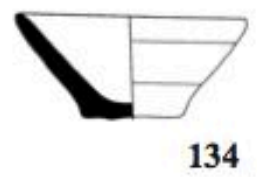



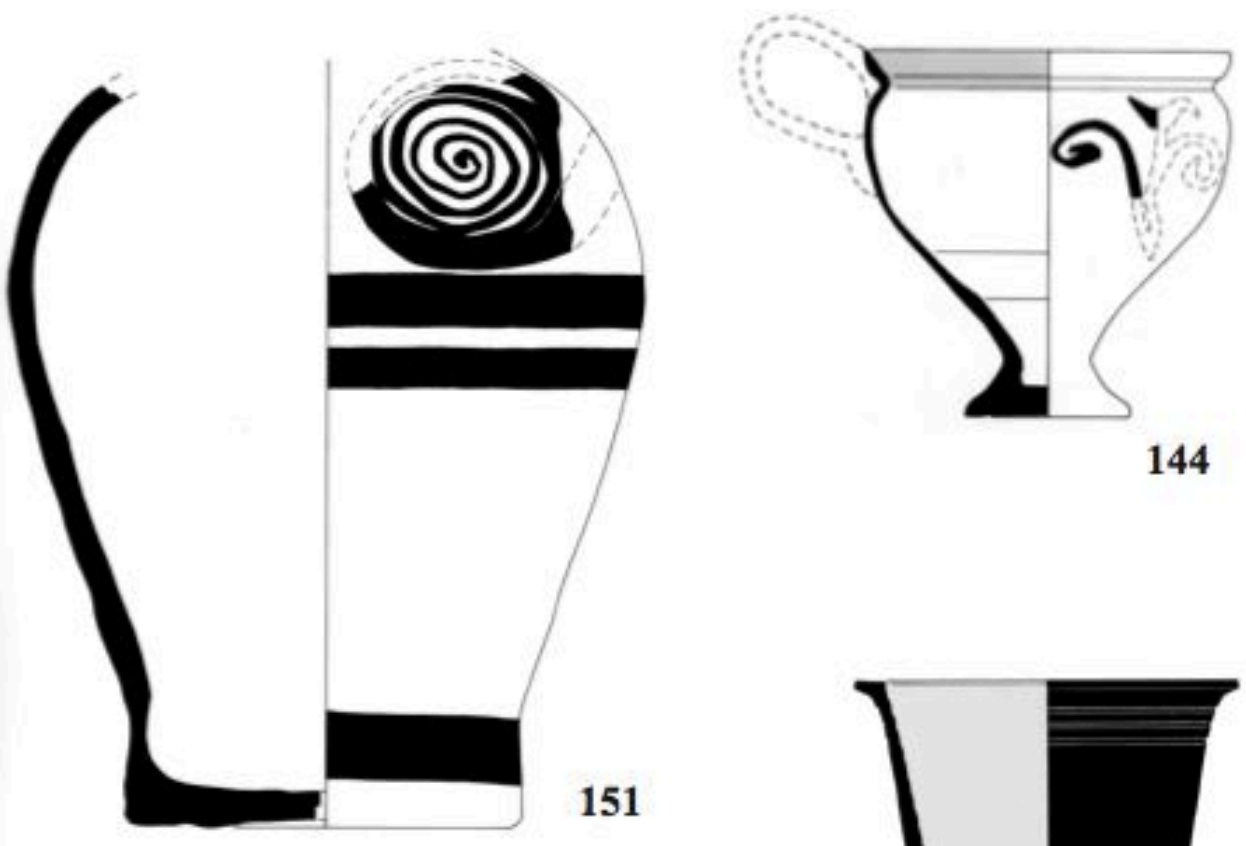

144

151

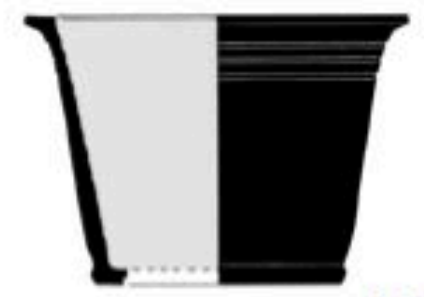

145
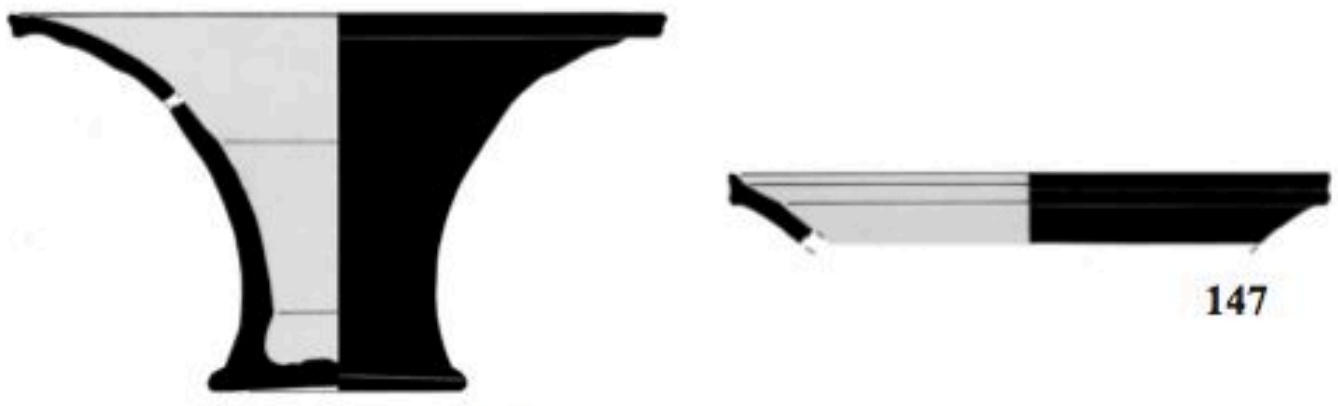

147
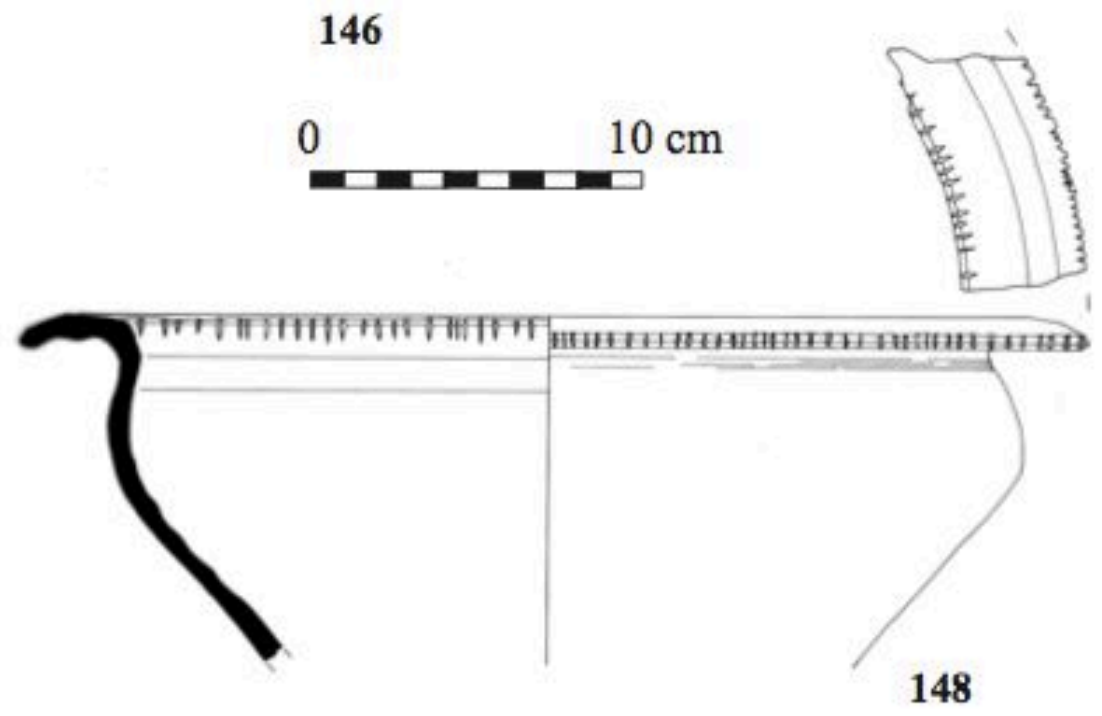

Fig.17 Catalogued pottery from feature 11. 


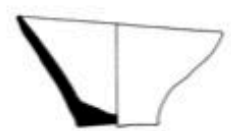

152

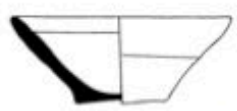

169

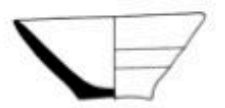

176

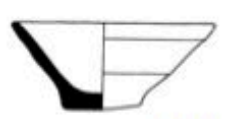

184

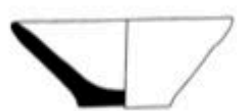

155

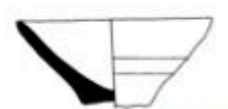

172

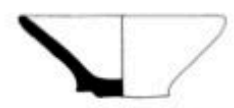

180

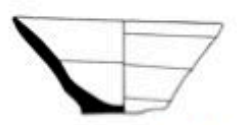

185

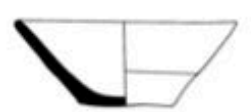

164

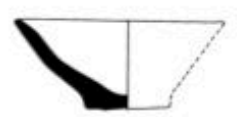

173

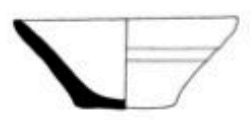

181

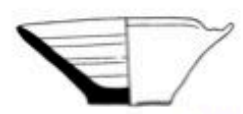

186

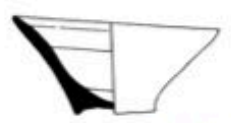

187

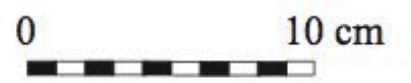

Fig.18 Catalogued pottery from feature 11. 


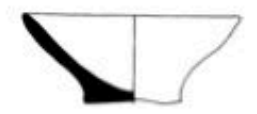

188
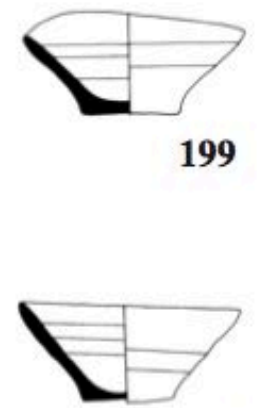

205

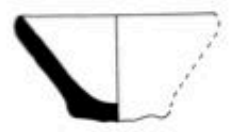

216

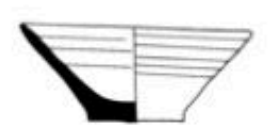

190
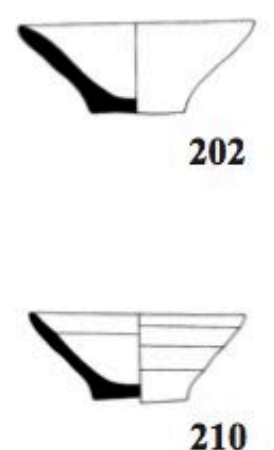

210

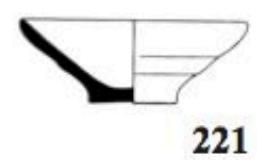

221

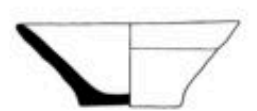

192

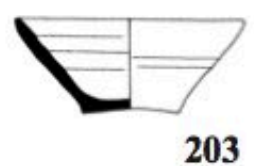

203

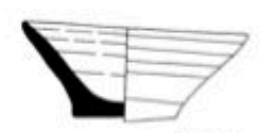

214

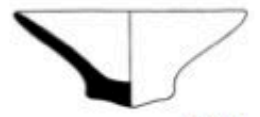

222

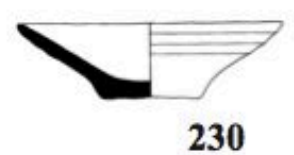

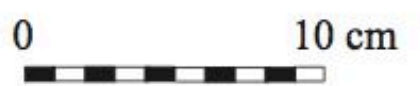

Fig.19 Catalogued pottery from feature 11. 


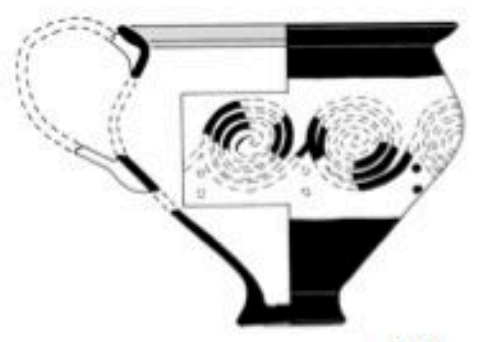

244

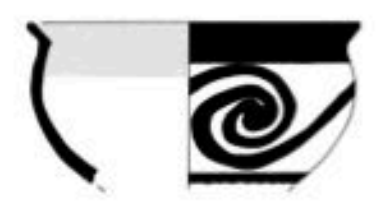

245

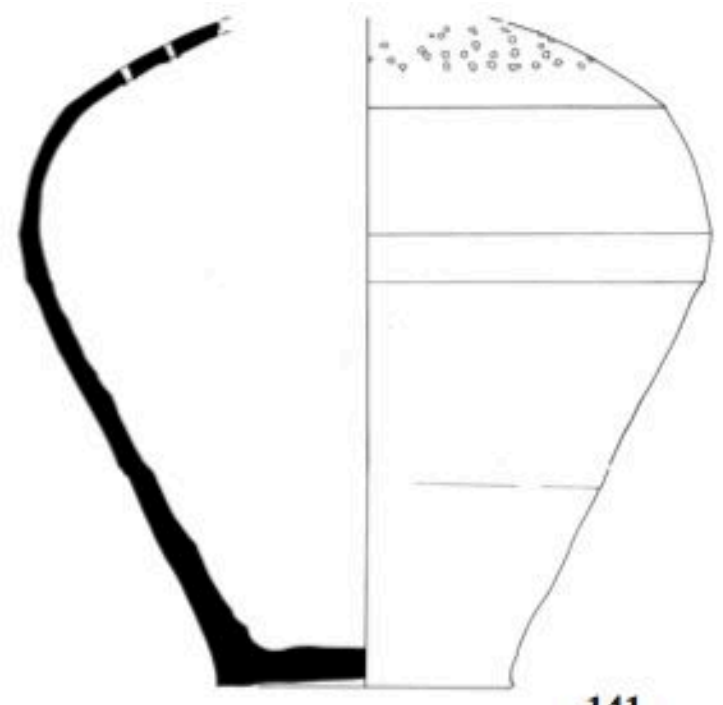

141

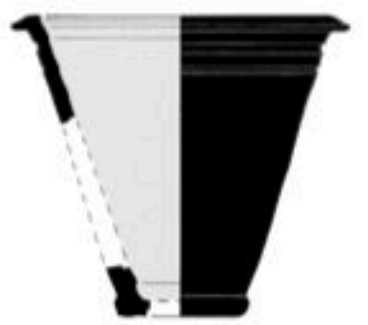

246

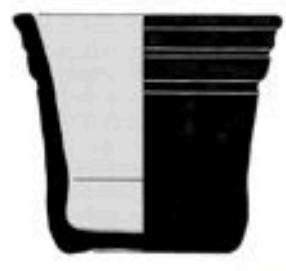

247

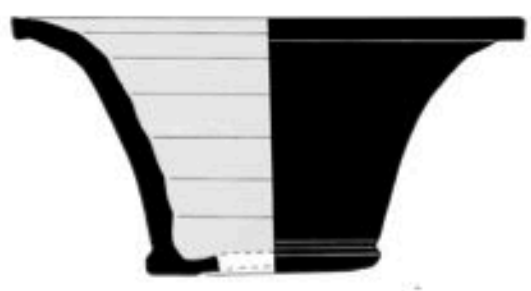

248

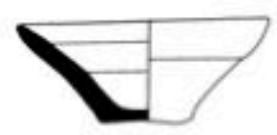

254

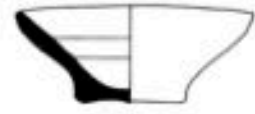

255

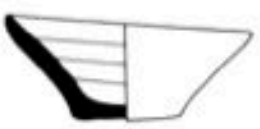

256

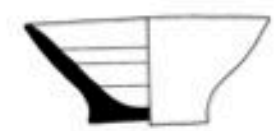

258
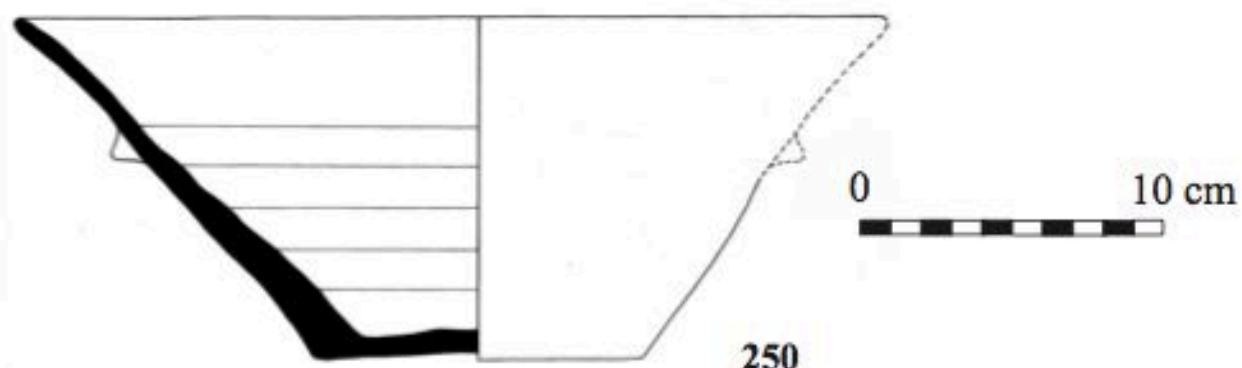

Fig.20 Catalogued pottery from features 25 and 9. 


\section{Conclusions}

The following discussion draws together the stratigraphic and ceramic evidence from Tholos and suggests some important implications of the Tholos finds, particularly for our understanding of the settlement, tombs and material culture of Neopalatial Kastri. The loose pottery from the excavation also points to the presence of Early Bronze Age, Minoanised late Prepalatial, and possibly Protopalatial pottery in the Tholos area. This indicates a much wider distribution of the material from Kastri's early phases, further inland than originally thought on the basis of the 1960s excavations. ${ }^{52}$ Moreover, the consistent presence of Neopalatial sherds in a red micaceous fabric amongst this loose material (but not the in situ ceramic assemblage) is interesting because this fabric usually relates to cooking and storage shapes and is common in LM I settlements on Kythera but not in the tombs. ${ }^{53}$ Its presence here may indicate settlement activity in the surrounding area, but is also a reflection of the effects of quarrying and erosion on top of the ridge. ${ }^{54}$ By contrast, very little post-Bronze Age pottery was found, all probably Archaic-Classical, and there is no evidence for Roman or Byzantine activity.

The in situ pottery from Tholos strongly suggests that these deposits are the remains of heavily truncated Neopalatial tombs. This conclusion is particularly robust in view of the distinctive character of the Neopalatial ceramic assemblages from the previously known Kastri tombs (FIG. 11). Conical cups are found en masse, as in the tombs excavated by Coldstream and Huxley but, more importantly, we find a range of other shapes paralleled mainly in the tombs at Kastri and rarely, if at all, in the contemporary settlement deposits: ring-handled basins, blossom bowls, kalathoi, tumblers, low-spouted jugs, ewers and conical bowls. While these links are striking, there are also some notable absences including very few, if any, of the braziers, lamps, censers or alabastra found in other tombs inventories at Kastri (FIG. 11). The possible relevance of this discrepancy is returned to below.

A closer examination of the excavated features themselves also corroborates their identification as tombs. These features fall into two categories: a) rectilinear rock cuttings, with a reddish-brown silty clay fill that covered conical cups, often stacked or inverted on a low dish and placed carefully on a small surface of water-worn pebbles, and other vessels. Immediately adjacent to these were b) less regularly shaped features, with more irregularly deposited ceramics. The link between these two categories is best understood at the junction between features 11 and 27. Here the lighter fill of 27 seems to spill over into 11 and the difference in depth leaves a small step down of c. $10 \mathrm{~cm}$ from 27 to 11 . This drop is very similar to the discrepancy in floor level noted at the

\footnotetext{
52 This material and its implications will be returned to in further study of the surface material of Kastri.

${ }^{53}$ Although this fabric is used for braziers and lamps found in tombs at Kastri, these shapes cannot be confirmed at Tholos, and certainly not in this fabric.

${ }^{54}$ Additional evidence of possible interspersed tombs and settlement on Kythera will be provided by the publication of the KIP survey data.
} 
entrances of secondary tomb chambers discovered in the 1960 s excavations. ${ }^{55}$ Feature 9 may relate to feature 5 in exactly the same way (i.e. with 5 representing a slightly deeper secondary chamber to 9), except that the remains are too shallow to preserve the relationship between them. Indeed, it is conceivable that features 21 and 25 are also related in exactly the same manner, though this is impossible to verify from the excavated sections.

So there are good reasons to conclude that the Tholos cuttings are indeed the remains of partially destroyed tombs. Two main factors would appear to go against this interpretation - the lack of human bone and the absence of definite examples of braziers, lamps, censers and alabastra - but both can be accounted for. The lack of bone may either be because all such remains have long since disintegrated or because these particular chambers were never used for human burial. In line with the first argument, we should note the poor condition of the pottery from Tholos (in comparison to the 1960s tombs), suggesting that conditions at this point on the ridge might, perhaps due to the existence of the trackway and resulting truncation, compaction and exposure to surface run-off and heat, also be particularly unsuitable for the preservation of human bone. In line with the second argument, we can note that although feature 11 shows exactly the sort of scattered distribution of pottery we might expect from communal tomb use, feature 5 appears surprisingly undisturbed. It is certainly not inconceivable that certain parts of the tomb were used as storage areas for funerary paraphernalia and not as loci for actual burial. Broadly comparable practices in contemporary Crete are witnessed at Myrtos Pyrgos ${ }^{56}$ and Archanes Phourni (Building 4) ${ }^{57}$ Indeed, the lack of any definite braziers, censers, lamps or alabastra $^{58}$ at Tholos may well reflect such a usage. These shapes are generally associated with the provision both of light and, more importantly, pleasant smells to mask the odour of decomposing human remains. In other words, their absence in the Tholos deposits may plausibly affirm the absence of actual burials from the chambers excavated.

The identification of the Tholos cuttings as tomb chambers has important implications with regard to local topography. It assumes that $1.2-2.0 \mathrm{~m}$ of bedrock (enough to extend the rock cavities up to a theoretical roof level) ${ }^{59}$ must have since disappeared. Prolonged erosion of this severity over the whole Kastri ridge is one possible explanation. However, the presence of significant patches of soil cover, abundant surface pottery of various periods (Early Bronze Age to Classical) across the whole area and the presence of well-preserved, rock-cut Classical cist tombs surviving not far from the Tholos excavation suggest on the contrary that there has instead been a much more selective loss of surface

\footnotetext{
55 especially Tomb E: Kythera, 223.

${ }^{56}$ G. Cadogan, 'Pyrgos, Crete, 1970-7', Archaeological Reports (1977-78), 70-84. See figs. 5 and 8, and p.73: "the LM I offerings of Pyrgos IV had been placed on the upper floor and eventually tumbled down over the burials below".

${ }^{57}$ Y. and E. Sakellarakis, Archanes: Minoan Crete in a New Light (Athens, 1997), 223-29. Building 4 is within the Phourni cemetery but was not used for burial. In this, and in the fact that it was in large part devoted to craft activities, it differs somewhat from the other contexts discussed here.

${ }_{58}$ Note the possible brazier in feature 5 and a possible alabastron in feature 21.

${ }^{59}$ This is based on the known heights of chambers in the $1960 \mathrm{~s}$ tombs $(1-1.6 \mathrm{~m})$ and the (at least) 20 $30 \mathrm{~cm}$ of bedrock on top of them.
} 
formations within this zone. In line with this, and with the traces of a scarp at the south end of the excavated area, it is probably more appropriate to postulate that a steep limestone bluff originally existed at this point on the ridge, curving away to the north-west to follow the modern paved road inland. Its disappearance here can be attributed to possible quarrying for local stone and/or the bulldozing of the track. Documentary evidence points to increased exploitation of the Palaiopolis area in the last two centuries and it is during this period that quarrying, road maintenance and, as a result, accelerating local erosion are likely to have been particularly intense. The name for this area, 'Tholos', in itself suggests some local familiarity with (and previous disturbance of) the ancient remains. ${ }^{60}$

The Tholos material can be added to an impressive array of known tombs that show that the marl limestone fringes of the Palaiopolis and Vothonas valleys that flank the Kastri ridge (probably inlets at this stage) ${ }^{61}$ were dominated by an extensive mortuary landscape. ${ }^{62}$ On the northern side of the Vothonas river, a tomb group has long been known at the very end of the Asprogas headland. Another tomb $(\mathrm{K})$ was found during well-digging, some $100 \mathrm{~m}$ inland and $3 \mathrm{~m}$ down into the Vothonas alluvium, and yet another group still further inland along the Asprogas ridge was excavated in the 1960s (B, E-H). On the south side of Vothonas, Coldstream and Huxley also excavated tombs originally found when the main road inland to Mitata was bulldozed (A, C-D, J). The Tholos remains now extend this southern distribution further up the Kastri ridge. Additional traces of such tombs are visible amongst the terraces on the southern, Palaiopolis, side of the same ridge. 63

Focussing now at a more detailed level, an explicit comparison of the material from the shallow remains at Tholos and other excavated tomb assemblages from the area is illuminating. In particular, the following comparisons are of significance:

\section{Dating}

Although all the Tholos material clearly belongs in LM I, it is difficult to specify with greater precision phases within this, i.e. LM IA and LM IB. This is largely because most of the parallels, particularly among the skeuomorphic vessels, come from the Kastri tombs rather than from stratified deposits in the settlement. A further obstacle to accurate dating is that there are very few useful off-island parallels, from Crete or elsewhere. Among the decorated finewares only two stemmed cups and one hemispherical cup are at all indicative of particular phases within LM I. Stemmed cup 144 (from feature 11) can be placed in LM IB on the grounds of both shape and decoration, while stemmed cup 244

\footnotetext{
${ }^{60}$ The Palaiopolis area was brought under more intensive cultivation by the inhabitants of several inland villages from c. $1800 \mathrm{AD}$ - see Leontsinis (n. 8), 36, 204,

${ }^{61}$ C. Frederick and A. Krahtopoulou pers. comm. Changes to the coastline around Kastri are part of an ongoing geoarchaeological study (as part of KIP) to be published elsewhere.

${ }^{62}$ Kythera, figs. 2-3.

${ }^{63}$ These have been documented by L. Preston and are part of a wider study of the Kytheran funerary landscape (as part of KIP) to be published elsewhere.
} 
and hemispherical cup 245 (both from feature 25) are good candidates for LM IA, judging by their dark-on-light spiral decoration.

Dating features 11 and 25 to LM IB and LM IA respectively on the basis of these three cups alone is far from satisfactory. We are also left with the problem of having no such finewares with which to pin down the date of feature 5. However, the rare decorated finewares are not the only material that might contribute to greater chronological resolution. The far more frequent conical cups also offer invaluable information. Recent work on Cretan pottery, from both the Protopalatial and Neopalatial periods, has shown that plain wares such as conical cups are very sensitive to change. ${ }^{64}$ In relation to the Tholos material this is a crucial point; we have noted above the consistent and striking differences between the conical cups of features 5 and 11 (with those from feature 25 comparable to those from 5). This is shown very clearly on the scatterplot of figure 12. The degree of difference between the conical cups of Types A and B is comparable to that seen amongst conical cup assemblages of different phases of Neopalatial Crete. ${ }^{65}$ It would seem that this points to the existence of two phases in the Tholos material, with the conical cups of features 5 and 25 representing one phase and those of feature 11 belonging to another. Moreover, unlike the finewares, there do exist parallels in the stratified settlement deposits of Kastri. Although Coldstream and Huxley do not publish many conical cups from their LM IA and LM IB deposits, they nonetheless describe clear differences in the shape, size and wall thickness of the conical cups from these two phases. Their comments are such that we can link the conical cups from Tholos features 5 and 25 with LM IA, and those from feature 11 with LM IB. This tallies with the dating suggested by the finewares mentioned above. It therefore seems likely, although not absolutely certain, that the two main contexts at Tholos, 5 and 11, belong to different phases of LMI, these being LM IA and LM IB respectively. One interesting implication is that burial customs on Kythera may have remained remarkably unchanged throughout the course of LM I66, despite the fact these two phases see quite significant change across the southern Aegean world as a whole. 67

\section{Number, size, shape and infilling of chambers}

The Tholos features seem to belong to several different tombs: feature 11 is probably a secondary chamber branching off a main area represented by feature 27 in one tomb; 5 a secondary chamber off 9 in another, and 25 and 21 are probably part of a third. The original entrances are difficult to infer, but $11 / 27$

\footnotetext{
${ }^{64}$ For the Protopalatial, see J.A. MacGillivray, Knossos: Pottery Groups of the Old Palace Period (BSA Studies 5; London, 1998). For the Neopalatial, and particularly MM III, see M.S.F. Hood, 'Back to Basics with Middle Minoan IIIB', in D. Evely, I. Lemos and S. Sherratt (eds.) Minotaur and Centaur. Studies in the archaeology of Crete and Euboea presented to Mervyn Popham (BAR Int.Series 638; Oxford, 1996), 10-16. Also, A. van de Moortel, The Transition from the Protopalatial to the Neopalatial Society in South-Central Crete: A Ceramic Perspective (Diss. Bryn Mawr College, 1997).

${ }^{65}$ Hood (n. 64); van de Moortel (n. 64); also C. Knappett and T.F. Cunningham, 'Three Neopalatial Deposits from Palaikastro, East Crete', (BSA, forthcoming).

${ }^{66}$ Although of course the settlement at Kastri does see significant changes between LM IA and IB, notably in the importation of finewares from Crete.

${ }^{67}$ J. Driessen and C. Macdonald, The Troubled Island. Minoan Crete Before and After the Santorini Eruption, (Aegaeum 17; Liège, 1997).
} 
may have been from the south, while 5/9 was perhaps from the east. This might suggest the original existence of a narrow bluff, perforated from different directions by tomb entrances. It is worth noting that the 1960 s tombs show great variation in the number of chambers each possesses. Their chambers also ranged from rounded to rectilinear and the Tholos features fit happily within the known range both in terms of size and shape. In terms of their primary fills, the Tholos cuttings (particularly 5 and 11) contained compact, reddish-brown deposits that were quite different from the existing surface soil of the top of the ridge and seem to have formed over a long period of time (see above, Excavated Features). Light (sometimes reddish), hard fills were also associated with the lower deposits of the 1960s tomb chambers, characterised by Neopalatial pottery and covered by darker, less compact Roman deposits. ${ }^{68}$

\section{Distribution of vessels within chambers and on pebbled areas}

In the 1960s tombs, pots were usually found smashed and scattered all over the floor (probably due to the repeated use of the structures, both during the Bronze Age and afterwards) and a very similar picture is presented by Tholos feature 11. In feature 5 however, a nest of closely packed pots was found in the middle of the cutting, and on a pebble surface in one corner, masses of conical cups were carefully stacked or inverted in a conical bowl. Indeed, three of the Tholos features $(5,11$ and 25) contained pebbled areas on which there were stored groups of conical cups. Similarly, in Tomb E, the excavators suggested that the whole chamber floor was covered with such pebbles, ${ }^{69}$ while a nest of conical cups was found in Tomb D, chamber g. ${ }^{70}$

\section{Types of pots and skeuomorphism}

At Tholos, each context has a very similar range and number of objects, indicating that the activities associated with burial ritual required a certain suite of artefacts (FIG. 11). In contrast, such shapes are not at all common from the areas of the settlement sampled during the 1960s excavations. These funerary pots are often skeuomorphs of metal and stone vessels and such imitation is a feature of Kytheran mortuary practice which deserves particular emphasis: perhaps the most exemplary form is the ring-handled basin, only found in ceramic in Kytheran tombs, but in metal in Cretan settlements. In contrast to Neopalatial Kythera, ceramic skeuomorphism of metal and stone shapes is rare in contemporary Crete, although this may be a feature of the lack of Neopalatial tomb assemblages on Crete and the relative paucity of skeuomorphing vessels in settlement contexts. The imitations might suggest that actual metal and stone vessels were quite rare on Kythera, but such a conclusion is not particularly convincing in the light of the silver cups and stone bowls at Kastri. ${ }^{71}$ In any case, Kytheran skeuomorphism reflects an unusually strong desire on the part of certain segments of the Kastri population to copy more status-laden media.

\footnotetext{
${ }^{68}$ Kythera, 220-26.

${ }^{69}$ Kythera, 223, fig.74. Note that the pebble deposit is described as 'much disturbed' and is portrayed as one or two discrete concentrations $(50+\mathrm{cm}$ across which is roughly the size of the Tholos patches) in the section drawing, so these may also have been isolated pebbled zones within the chamber.

${ }^{70}$ Kythera, pl. 65d.

${ }^{71}$ e.g. Kythera - silver cups: pls. 59: 23-4, 60: 23-4; stone vessels: pls. 61: 37; 62: 6, 62, 152, 154, 346, $348 ; 69: 39 ; 82: 8,9,11 ; 83: 6-7,13-16$.
} 


\section{Comparable off-island LMI tombs and grave goods}

Beyond Kythera, there are few good parallels for the Kastri tombs. The most relevant comparanda are chamber tombs from Knossos (Mavro Spelio V, IX and $\mathrm{X}$ in particular) ${ }^{72}$ and Poros, ${ }^{73}$ but the similarities are general ones. Taken as a whole, the LMI Cretan burials suggest great diversity in mortuary practice, with the use of pithos burials, chamber tombs and built tombs, as well as both larnakes and simple inhumation. ${ }^{74}$ In contrast, the mainly multi-chambered, rock-cut tombs (without evidence to date for larnax use) at Kastri represent a single, specific strategy, albeit with room for diversity in terms of size and architectural elaboration. In fact, the combination of relative on-island uniformity and few close off-island parallels cannot be stressed enough. This is also true of grave goods. There are some broad parallels with Crete, including the deployment of large numbers of conical cups (or cups in general) and the consistent presence of pouring vessels. We should probably see these shapes as reflecting both the general importance of food and drink consumption as a display mechanism in Neopalatial society and the specific role of feasting ceremonies within burial rites. However, the consistent presence of a special set of ceramic vessels (which were not common in the Kastri settlement) in all the Kastri tombs is unique to the island. This consistent practice is further emphasised by the degree of skeuomorphism of metal and stone shown by the pottery, which suggests that not only were certain shapes being prioritised to an unusual extent but, by a process of skeuomorphic allusion, so too was an ideology of certain higher value materials, even if the actual counterparts in metal and stone were not always present.

To summarise, this report has outlined the significance of finds from the Tholos excavations within their broader cultural context at Kastri, on Kythera and in the wider Aegean world. As such, it forms one part of ongoing research into the dynamics of Bronze Age Kythera. The finds and their interpretation contribute to a richer understanding not only of the immediate funerary landscape around Kastri, but also of the peculiarities of Kytheran burial strategies and material culture in the Neopalatial period.

\footnotetext{
${ }^{72}$ E.J. Forsdyke, 'The Mavrospelio Cemetery at Knossos', BSA 28 (1926-7), 243-96.

${ }^{73}$ N. Dimopoulou, 'The Neopalatial Cemetery of the Knossian Harbour-Town at Poros: Mortuary Behaviour and Social Ranking,' in I. Kilian-Dirlmeier (ed.), Eliten in der Bronzezeit (Bonn, 1999), $27-$ 36. She notes the "overall similarities with the Kythera tombs with multiple chambers" (p. 28), as well as the ubiquity of the conical cup in the Poros tombs (p. 29).

${ }^{74}$ For an important recent discussion, see L. Preston, 'A Mortuary Approach to Cultural Interaction and Political Dynamics in Late Minoan II-IIIB’ (unpublished PhD diss., UCL 2001), chapter 3.
} 

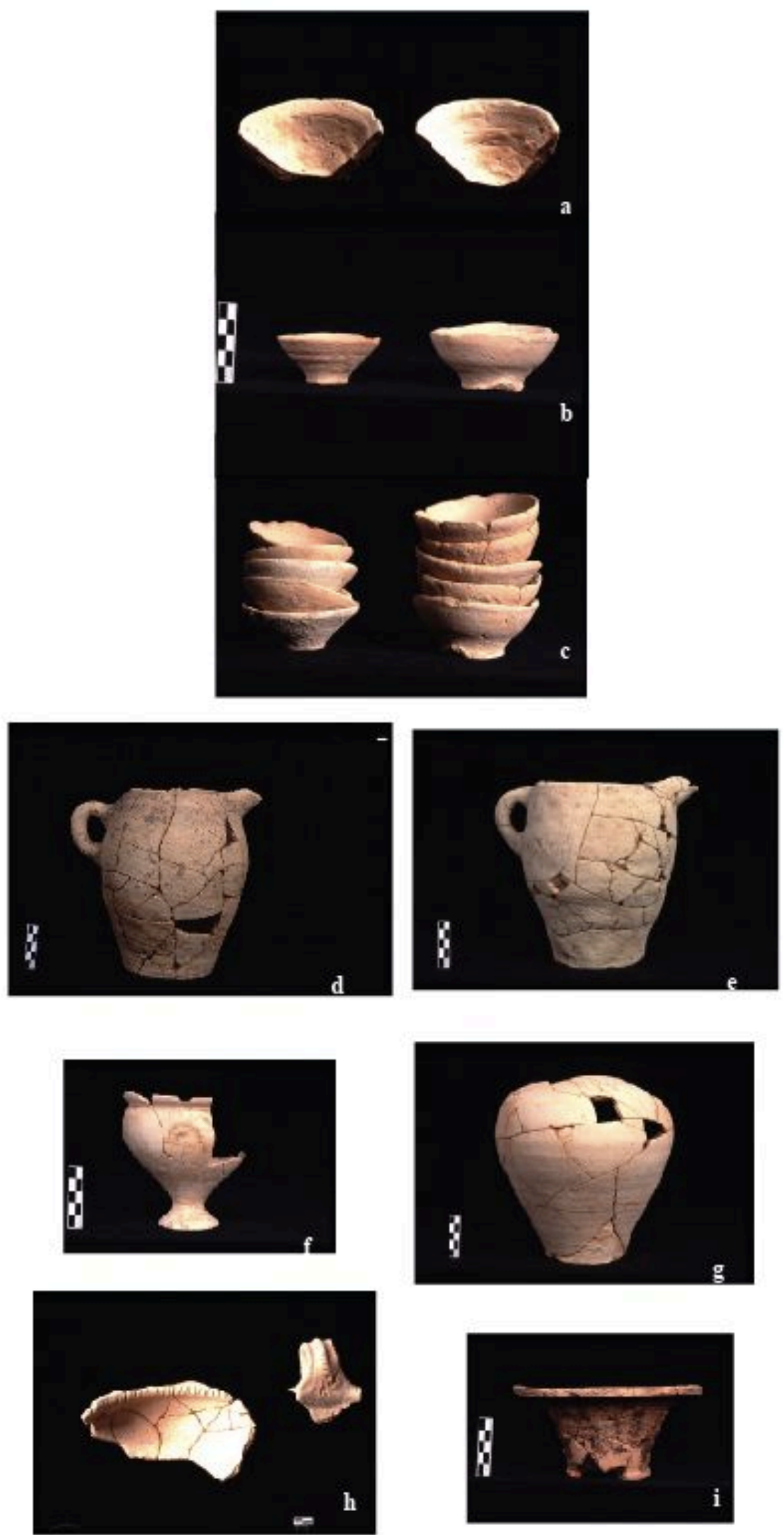

Plate 1 - a) conical cups: coil seams on interior, b) conical cups: Type A (right) and Type B (left), and c) conical cups: stacks of Type A (right) and Type B (left), d) low-spouted jug (cat.no. 9), e) low-spouted jug (cat.no. 10), f) stemmed cup (cat.no. 144), g) perforated jug (cat.no. 141), h) ring-handled basin (cat.no. 5), i) kalathos (cat.no. 248). 

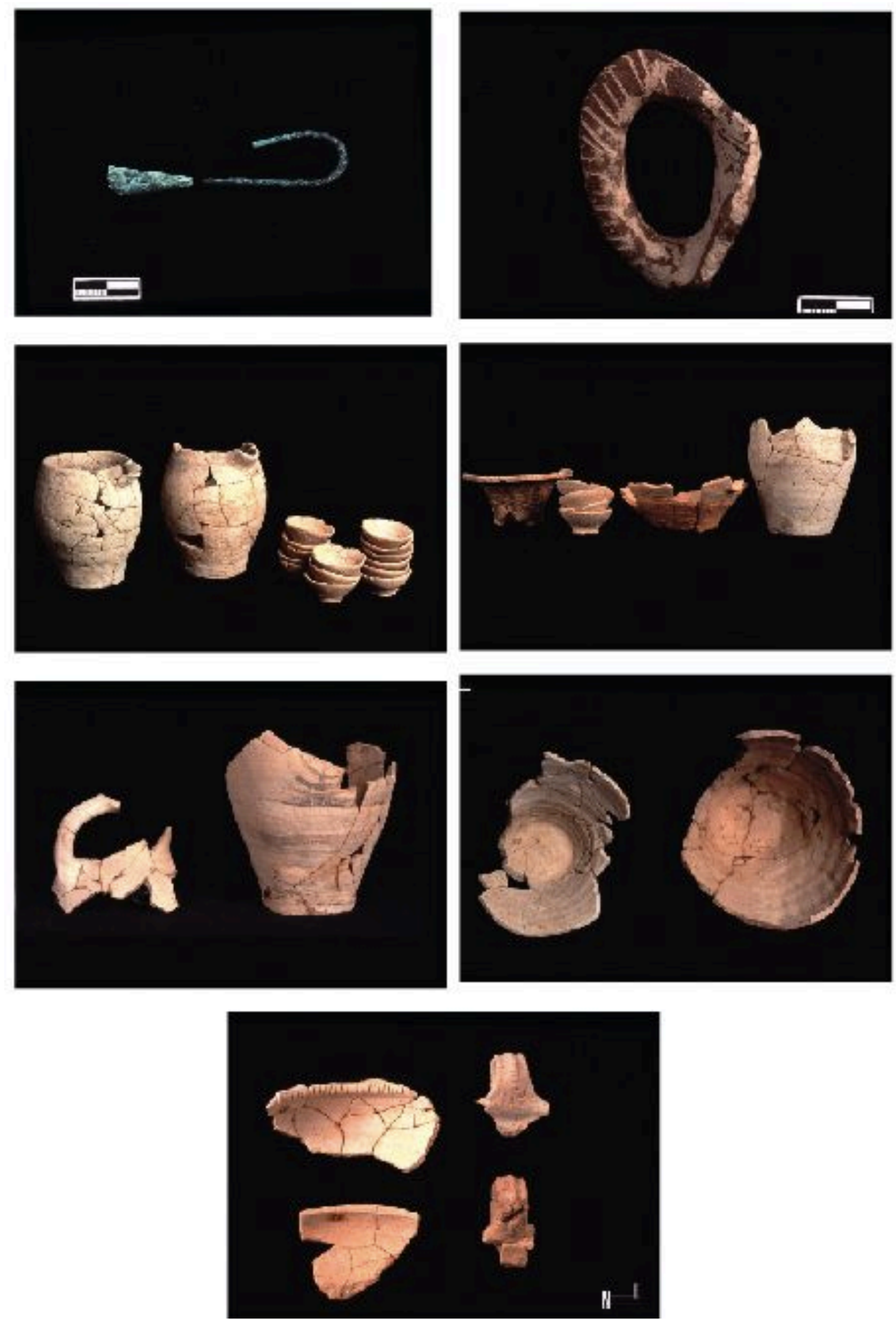

Plate 2 - a) copper/bronze wire, b) EBA handle with incised decoration, c) pottery from feature 5, d) pottery from feature 25, e) ewer (cat.no. 12), f) ewers: manufacture evidence on interior (cat.no 11-left, cat.no. 12-right) g) ring-handled basins (cat.no.5-top, cat.no.6-bottom) 


\section{Summary List of Contexts Numbers Used in the Field}

\section{Context Definition}

corner of site

fill of 2, gravel backfill of 1999 test

marl limestone bedrock

cut, rectangular pit/feature, SW of 11

deposit under 1 and above 2 in SW section of site, possible older soil horizon

fill of feature 9

fill of feature 5

cut, irregular pit/feature, $\mathrm{N}$ of 5

fill of feature 11

cut, rectangular pit/feature, NE of 5

fill of feature 9 (same as 7)

fill of feature 11 (same as 10)

fill of feature 15

cut, pit/?posthole within feature 9 (appears to be overlain by 7)

fill of feature 11 (same as 10; to be relabelled)

fill of feature 5 , pebble floor in S corner under conical cups

cut, bulldozed road-cut

deposit, soil accumulation abutting SE side of (or fill of) feature 29

fill of feature 21

cut, irregular pit/feature (partially excavated)

fill (part of 19; to be relabelled)

fill of feature 11, pebble floor in N corner under conical cups

fill of feature 25

cut, pit/feature (partially excavated)

fill of feature 27

cut, feature/pit immediately SW of 11 


\begin{tabular}{|l|l|}
\cline { 2 - 2 } 28 & deposit/fill, soil in depression SE of 11 and NW of 33 \\
\cline { 2 - 2 } 29 & $\begin{array}{l}\text { cut?, linear break in bedrock across track; deliberate cutting or natural } \\
\text { bluff }\end{array}$ \\
\cline { 2 - 2 } 30 & deposit, small charcoal patch S of pit 11, probably recent \\
\cline { 2 - 2 } 31 & deposit, small charcoal patch S of pit 11, probably recent \\
\cline { 2 - 2 } 33 & deposit/fill, in depression SE of 33 and NW of 29 (probably same as 28) \\
\cline { 2 - 2 } 32 & line of stones placed on 28/32 (possibly to consolidate soil) \\
\hline
\end{tabular}

\section{Major Context Relationships}

Feature $5 \quad$ main fill 8

pebble floor 17

Feature 9 main fill 7 (other fill 12 is part of 7)

appears to truncate 15

Feature 11 main fill 10 (other fills $13 \& 16$ are part of 10) pebble floor 23

Feature 15 fill 14

appears to be truncated by 9

Feature $21 \quad$ fill 20

Feature 25 main fill 24

traces of pebble floor (not given a context number)

Feature $27 \quad$ fill 26

probably part of larger feature with 11

Feature 29 exact relationships uncertain

fill/abutting deposit 19 (and 22 which is the same as 19) 


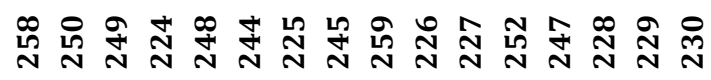

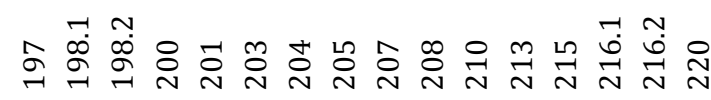

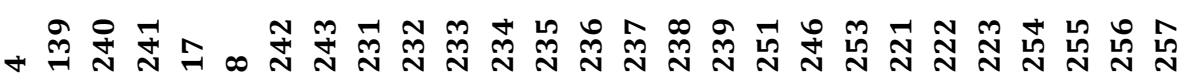

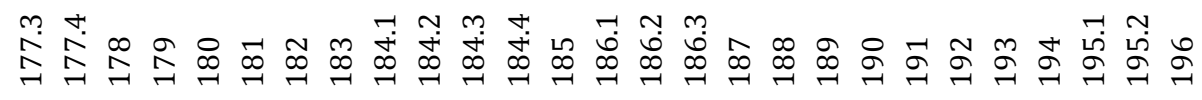

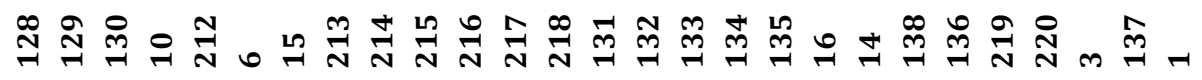

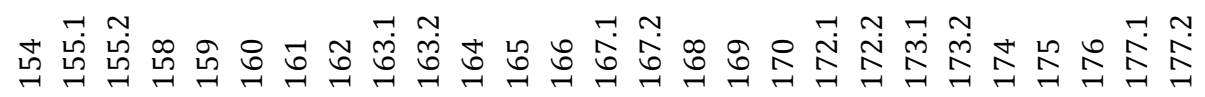

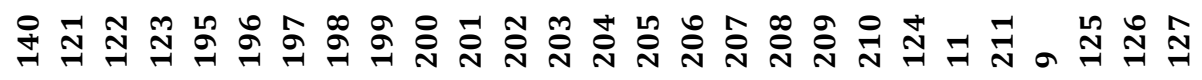

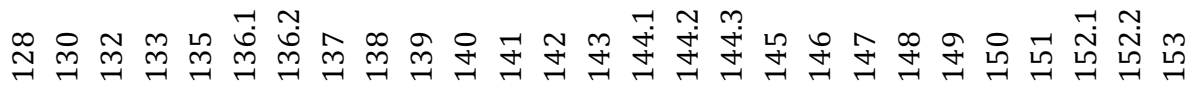

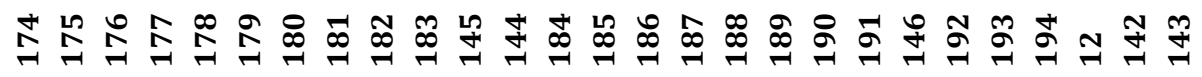

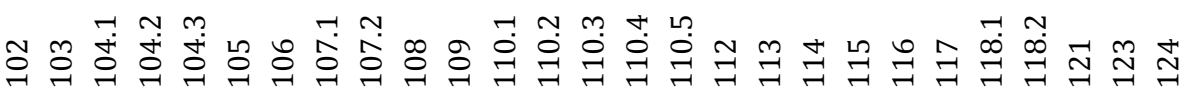

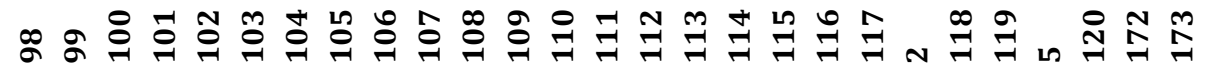

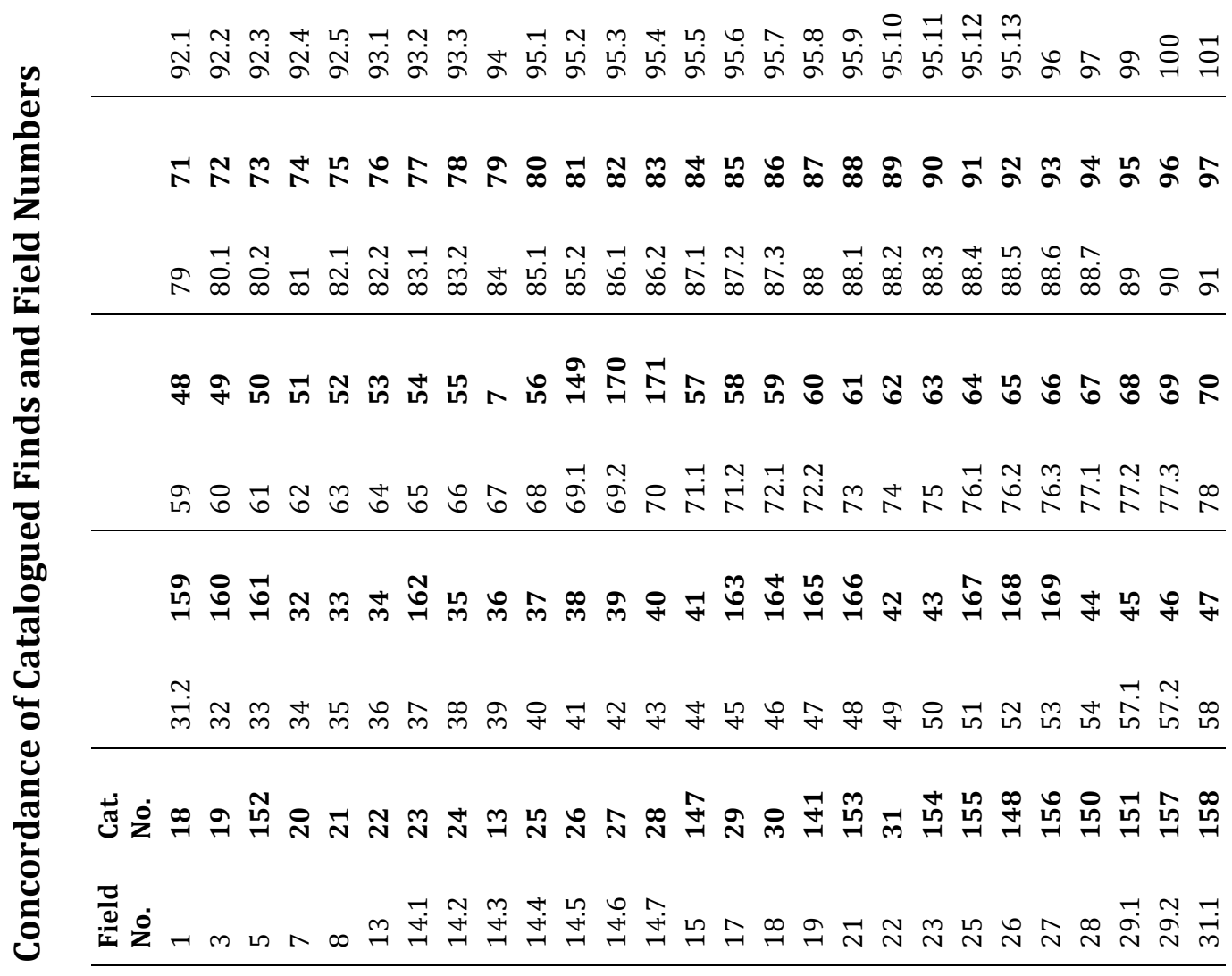

